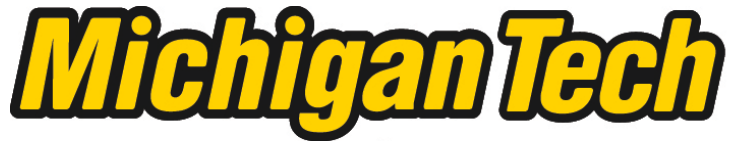 \\ Michigan Technological University Create the Future Digital Commons @ Michigan Tech
}

Dissertations, Master's Theses and Master's Reports - Open

Dissertations, Master's Theses and Master's

Reports

2011

\section{Fading pdf of free-space optical communication system with pointing error}

Rui Liao

Michigan Technological University

Follow this and additional works at: https://digitalcommons.mtu.edu/etds

Part of the Electrical and Computer Engineering Commons

Copyright 2011 Rui Liao

\section{Recommended Citation}

Liao, Rui, "Fading pdf of free-space optical communication system with pointing error", Dissertation, Michigan Technological University, 2011.

https://doi.org/10.37099/mtu.dc.etds/64

Follow this and additional works at: https://digitalcommons.mtu.edu/etds

Part of the Electrical and Computer Engineering Commons 
FADING PDF OF FREE-SPACE OPTICAL COMMUNICATION SYSTEM WITH

POINTING ERROR

By

RUI LIAO

A DISSERTATION

Submitted in partial fulfillment of the requirements for the degree of DOCTOR OF PHILOSOPHY

(Electrical Engineering)

MICHIGAN TECHNOLOGICAL UNIVERSITY

2011

(C) 2011 Rui Liao 

This dissertation, "Fading Pdf of Free-Space Optical Communication System with Pointing Error," is hereby approved in partial fulfillment of the requirements for the Degree of DOCTOR OF PHILOSOPHY IN ELECTRICAL ENGINERRING.

Department of Electrical and Computer Engineering

Signatures:

Dissertation Advisor

Dr. Zhijun Zhao

Committee

Dr. Daniel R. Fuhrmann

Dr. Haiying Liu

Dr. Zhuo Feng

Department Chair

Dr. Daniel R. Fuhrmann

Date 



\section{Dedication}

To My Wife Jia Yang and My Son Ethan Y. Liao. 


\section{Contents}

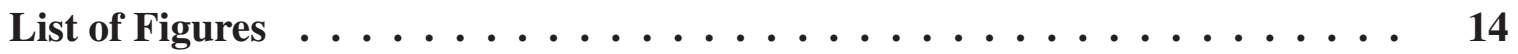

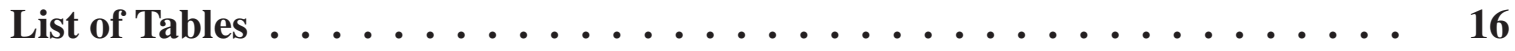

Acknowledgments $\ldots \ldots \ldots \ldots \ldots \ldots \ldots \ldots \ldots \ldots \ldots$

Abstract .......................... 19

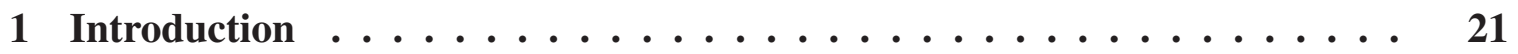

1.1 Motivation . . . . . . . . . . . . . . . . . . . . 22

1.2 Background Review . . . . . . . . . . . . . . . . . . . . . 24

1.3 Mathematical Fading Pdf Models Review _ . . . . . . . . . . 26

1.3.1 Log-normal (LN) Fading Pdf Model . . . . . . . . . . . . . 26

1.3.2 K Fading Pdf Model $\ldots \ldots \ldots \ldots$

1.3.3 IK Fading Pdf Model . . . . . . . . . . . . . . . . . . . . . 29

1.3.4 LNME Fading Pdf Model . . . . . . . . . . . . . . . . . . 31

1.3.5 Beckmann (LNMR) Fading Pdf Model . . . . . . . . . . . . 32

1.3.6 Gamma-Gamma (GG) Fading Pdf Model . . . . . . . . . . 34

1.4 Summary of Key Results . . . . . . . . . . . . . . . . . . . . . 35 
1.5 Organization of Dissertation $\ldots \ldots \ldots \ldots \ldots$

2 Fading Pdf for FSO Communication System with Pointing Error . . . . . 41

2.1 Introduction . . . . . . . . . . . . . . . 41

2.2 Analytical Method . . . . . . . . . . . . . . . . 44

2.3 Pointing Error Models . . . . . . . . . . . . . . . . . 46

2.3.1 Fast-tracking Residual Error . . . . . . . . . . . . . . 47

2.3.2 Beam Wander Plus Transceiver Induced Extra Pointing Error . . . 48

2.3.2.1 Beam Wander ............. . . . 50

2.3.2.2 Two Dimensional Gaussian Random Model . . . . . . . 50

2.3.2.3 Two Dimensional Uniform Random Model . . . . . . . 51

2.3.2.4 Sine Sway Model . . . . . . . . . . . . . 52

2.4 Simulation Setup . . . . . . . . . . . . . . . . 53

2.5 Simulation Results . . . . . . . . . . . . . . . . . 55

2.5.1 Channel Information ................ 55

2.5.2 Overall Fading Pdf . . . . . . . . . . . . . . 56

2.5.2.1 $L=1.6 \mathrm{Km}, C_{n}^{2}=5 \times 10^{-15} \mathrm{~m}^{-2 / 3}$ Channel . . . . . 56

2.5.2.2 $L=4 \mathrm{Km}, C_{n}^{2}=5 \times 10^{-15} \mathrm{~m}^{-2 / 3}$ Channel . . . . . . 58

2.5.2.3 L $L=0.4 \mathrm{Km}, C_{n}^{2}=1 \times 10^{-13} \mathrm{~m}^{-2 / 3}$ Channel . . . . . . 61

2.5.3 Average BER and Outage Probability . . . . . . . . . 63

2.6 Conclusion ............................ 69

3 Fading Pdf with Beam Diverging Angle $\ldots \ldots \ldots \ldots$ 
3.1 Introduction . . . . . . . . . . . . . . 73

3.2 Simulation Setup . . . . . . . . . . . . . . . . . 77

3.3 Simulation Results . . . . . . . . . . . . . . . . . 79

3.3.1 Fast-tracked Fading Pdf . . . . . . . . . . . . . . . . . . . . . . 79

3.3.2 Overall Fading Pdf . . . . . . . . . . . . . . 80

3.4 Conclusion ............................ 91

4 Fading pdf Models Comparison $\ldots \ldots \ldots$

4.1 Introduction . . . . . . . . . . . . . . . . 93

4.2 Comparison with Simulation Data . . . . . . . . . . . . . . . 94

4.2.1 Beam Wander Only Pointing Error . . . . . . . . . . . . 96

4.2.2 General Pointing Error . . . . . . . . . . . . . . . . . . 99

4.3 Average BER and Outage Probability . . . . . . . . . . . . . . . . 102

4.4 Discussion . . . . . . . . . . . . . . . . . . 107

4.5 Conclusions . . . . . . . . . . . . . . . . . 108

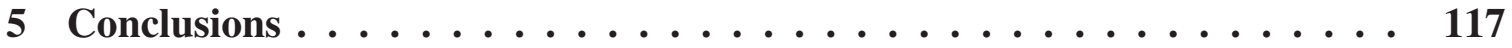

5.1 Summary of Dissertation . . . . . . . . . . . . . 117

5.2 Summary of Key Results . . . . . . . . . . . . . . . . . . . . . . . 121

5.3 Future Work . . . . . . . . . . . . . . . . . . 122

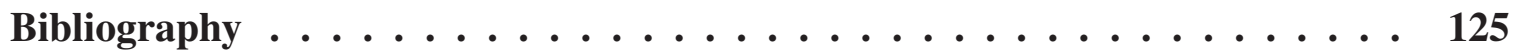

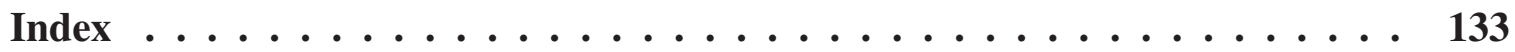




\section{List of Figures}

2.1 Random walk of the beam center. . . . . . . . . . . . . . .

2.2 Comparison for the analytical curve with the direct wave-optics simulation curve. $C_{n}^{2}=5 \times 10^{-15} \mathrm{~m}^{-2 / 3} . L=1.6 \mathrm{Km}$. Gaussian extra pointing error model. $\sigma_{b}=0.58 \mathrm{~cm} . \sigma_{p}=2.5 \mathrm{~cm} . \sigma_{d}=2.57 \mathrm{~cm} . \Delta x=\Delta y=0 \mathrm{~cm} . d_{x}=$ $2.5 \mathrm{~mm}$. Coherence radius is $4.2 \mathrm{~cm}$. Rytov variance is 0.24 . $1024 \times 1024$. $0.56 \mathrm{M}$ iterations. . . . . . . . . . . . . . . . .

2.3 Comparison for the analytical curve with the direct wave-optics simulation curve. $C_{n}^{2}=5 \times 10^{-15} \mathrm{~m}^{-2 / 3} . L=1.6 \mathrm{Km}$. Gaussian extra pointing error model. $\sigma_{b}=0.58 \mathrm{~cm} . \sigma_{p}=2.5 \mathrm{~cm} . \sigma_{d}=2.57 \mathrm{~cm} . \Delta x=\Delta y=2.5 \mathrm{~cm} . d_{x}=$ $2.5 \mathrm{~mm}$. Coherence radius is $4.2 \mathrm{~cm}$. Rytov variance is $0.24 .1024 \times 1024$. $0.70 \mathrm{M}$ iterations. . . . . . . . . . . . . .

2.4 Comparison for the analytical curve with the direct wave-optics simulation curve. $C_{n}^{2}=5 \times 10^{-15} \mathrm{~m}^{-2 / 3} . L=4 \mathrm{Km}$. Gaussian tracking residual error model. $\sigma_{d}=2 \mathrm{~cm} . \Delta x=\Delta y=0 \mathrm{~cm} . d_{x}=1.6 \mathrm{~mm}$. Coherence radius is 2.4cm. Rytov variance is $1.27 .1024 \times 1024$. $0.49 \mathrm{M}$ iterations. . . . . . . 
2.5 Comparison for the analytical curve with the direct wave-optics simulation curve. $C_{n}^{2}=5 \times 10^{-15} \mathrm{~m}^{-2 / 3} . L=4 \mathrm{Km}$. Gaussian extra pointing error model. $\sigma_{b}=2.29 \mathrm{~cm} . \quad \sigma_{p}=1 \mathrm{~cm} . \quad \sigma_{d}=2.5 \mathrm{~cm} . \quad \Delta x=\Delta y=0 \mathrm{~cm} . \quad d_{x}=$ $2.5 \mathrm{~mm}$. Coherence radius is $2.4 \mathrm{~cm}$. Rytov variance is $1.27 .1024 \times 1024$.

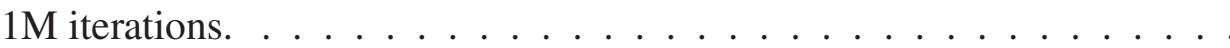

2.6 Comparison for the analytical curve with the direct wave-optics simulation curve. $C_{n}^{2}=5 \times 10^{-15} \mathrm{~m}^{-2 / 3} . L=4 \mathrm{Km}$. Uniform random model and sine sway model. $\sigma_{b}=2.29 \mathrm{~cm} . s=2 \mathrm{~cm} . d_{x}=2.5 \mathrm{~mm}$. Coherence radius is 2.4cm. Rytov variance is $1.27 .1024 \times 1024.1 \mathrm{M}$ iterations. . . . . . . .

2.7 Comparison for the analytical curve with the direct wave-optics simulation curve. $C_{n}^{2}=1 \times 10^{-13} \mathrm{~m}^{-2 / 3} . L=0.4 \mathrm{Km}$. Gaussian extra pointing error. $\sigma_{b}=0.32 \mathrm{~cm} . \sigma_{p}=1.25 \mathrm{~cm} . \sigma_{d}=1.29 \mathrm{~cm} . \Delta x=\Delta y=0 \mathrm{~cm} . d_{x}=0.625 \mathrm{~mm}$. Coherence radius is $1.6 \mathrm{~cm}$. Rytov variance is $0.37 .2048 \times 2048.0 .41 \mathrm{M}$ iterations. . . . . . . . . . . . . . . . . . .

2.8 Comparison for the averaged BER among simulation, analytical method, GG model and LN model. $C_{n}^{2}=5 \times 10^{-15} \mathrm{~m}^{-2 / 3} . L=1.6 \mathrm{Km}$. Gaussian random model. $\sigma_{b}=0.58 \mathrm{~cm} . \quad \sigma_{p}=2.5 \mathrm{~cm} . \quad \sigma_{d}=2.57 \mathrm{~cm} . \Delta x=\Delta y=$ $2.5 \mathrm{~cm} . d_{x}=2.5 \mathrm{~mm}$. Coherence radius is $4.2 \mathrm{~cm}$. Rytov variance is 0.24 . $1024 \times 1024.0 .70 \mathrm{M}$ iterations. . . . . . . . . . . . . . . . 67

3.1 Fast-tracked on-axis and off-axis fading pdfs for focused beam. . . . . . . 81

3.2 Fast-tracked on-axis and off-axis fading pdfs for diverged beam. . . . . . 82 
3.3 Fast-tracked beam profile and fading variance for focused beam. . . . . .

3.4 Fast-tracked beam profile and fading variance for diverged beam. . . . . .

3.5 Overall fading pdfs comparison for focused beam. . . . . . . . . . . .

3.6 Overall fading pdfs comparison for diverged beam. . . . . . . . . .

4.1 Fading pdf models comparison. Beam wander only pointing error. . . . .

4.2 Fading pdf models comparison. Gaussian extra pointing error. $C_{n}^{2}=1 \times$ $10^{-13} \mathrm{~m}^{-2 / 3} . L=0.4 \mathrm{Km} . \Delta x=\Delta y=0 \mathrm{~cm} . \sigma_{b}=0.32 \mathrm{~cm} . \sigma_{p}=1.25 \mathrm{~cm}$. $\sigma_{d}=1.29 \mathrm{~cm} . d_{x}=0.625 \mathrm{~mm}$. Coherence radius is $1.6 \mathrm{~cm}$. Rytov variance

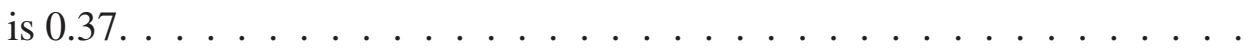

4.3 Fading pdf models comparison. Gaussian extra pointing error model. $C_{n}^{2}=$ $5 \times 10^{-15} \mathrm{~m}^{-2 / 3} . L=1.6 \mathrm{Km} . \sigma_{b}=0.58 \mathrm{~cm} . \sigma_{p}=2.5 \mathrm{~cm} . \sigma_{d}=2.57 \mathrm{~cm}$. Coherence radius is $4.2 \mathrm{~cm}$. Rytov variance is 0.24

4.4 Fading pdf models comparison. Fast-tracking residual error model. $C_{n}^{2}=$ $5 \times 10^{-15} \mathrm{~m}^{-2 / 3} . L=4 \mathrm{Km} . \Delta x=\Delta y=0 \mathrm{~cm} . \quad \sigma_{d}=2 \mathrm{~cm} . \quad d_{x}=1.6 \mathrm{~mm}$. Coherence radius is $2.4 \mathrm{~cm}$. Rytov variance is 1.27 . . . . . . . . . .

4.5 Fading pdf models comparison. Gaussian extra pointing error model. $C_{n}^{2}=$ $5 \times 10^{-15} \mathrm{~m}^{-2 / 3} . L=4 \mathrm{Km} . \Delta x=\Delta y=0 \mathrm{~cm} . \quad \sigma_{b}=2.29 \mathrm{~cm} . \quad \sigma_{p}=1 \mathrm{~cm}$. $\sigma_{d}=2.5 \mathrm{~cm} . d_{x}=2.5 \mathrm{~mm}$. Coherence radius is $2.4 \mathrm{~cm}$. Rytov variance is

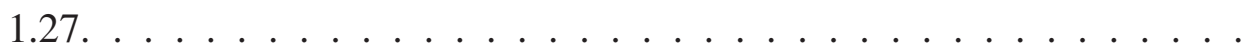

4.6 Average BER comparison. $L=1.6 \mathrm{Km} . C_{n}^{2}=5 \times 10^{-15} \mathrm{~m}^{-2 / 3}$. Gaussian extra pointing error. $\sigma_{b}=0.58 \mathrm{~cm} . \sigma_{p}=2.5 \mathrm{~cm} . \sigma_{d}=2.57 \mathrm{~cm} . \quad \ldots$. 
4.7 Average BER comparison. $L=4 \mathrm{Km} . C_{n}^{2}=5 \times 10^{-15} \mathrm{~m}^{-2 / 3}$. Beam wander only pointing error and Gaussian extra pointing error. . . . . . . . . . 115 


\section{List of Tables}

2.1 Rytov variance and coherence radius. . . . . . . . . . . . . . . 54

2.2 Simulation parameters for channel information. . . . . . . . . 54

2.3 Simulation parameters for overall fading pdf. $\ldots \ldots \ldots \ldots \ldots$

2.4 Fast-tracked fading pdf variance for point-like $0 \mathrm{~cm}$ aperture $\ldots \ldots \ldots$

$2.510 \log _{10}(A)$ requires to achieve $\mathrm{BER}=1 \times 10^{-5} \ldots \ldots \ldots \ldots$

2.6 Probability (\%) for $10 \mathrm{~dB}$ outage. $L=1.6 \mathrm{Km} . C_{n}^{2}=5 \times 10^{-15} \mathrm{~m}^{-2 / 3}$. Gaussian random pointing error model. $\Delta x=\Delta y=2.5 \mathrm{~cm}$, and $\sigma_{p}=2.5 \mathrm{~cm}$ in

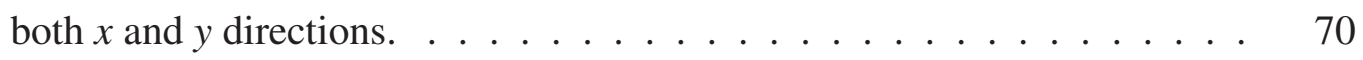

3.1 Simulation parameters for focused beam. $L / F=1 \ldots \ldots \ldots \ldots$

3.2 Simulation parameters for diverged beam. $L / F=-1 \ldots \ldots \ldots \ldots \ldots$

3.3 Beam radius $W$ and beam wander standard deviation $\sigma_{w}$ for focused beam. $L / F=1 \ldots \ldots \ldots \ldots \ldots \ldots \ldots \ldots \ldots \ldots \ldots \ldots \ldots$

3.4 Beam radius $W$ and beam wander standard deviation $\sigma_{w}$ for diverged beam. $L / F=-1 \ldots \ldots \ldots \ldots \ldots \ldots \ldots \ldots \ldots \ldots \ldots \ldots$

4.1 Rytov variance and coherence radius. . . . . . . . . . . . . 97

4.2 Simulation parameters for beam wander only pointing error case. . . . . 97 
4.3 Rytov variance and coherence radius. . . . . . . . . . . . . . . . 100

4.4 Simulation parameters for channel information in general pointing error case. 100

4.5 Direct simulation parameters for overall fading with general pointing error case............................... 100

4.6 Probability (\%) for $10 \mathrm{~dB}$ outage. $L=1.6 \mathrm{Km} . C_{n}^{2}=5 \times 10^{-15} \mathrm{~m}^{-2 / 3}$.

Gaussian extra pointing error model. $\Delta x=\Delta y=0 \mathrm{~cm}$, and $\sigma_{b}=0.58 \mathrm{~cm}$, $\sigma_{p}=2.5 \mathrm{~cm}, \sigma_{d}=2.57 \mathrm{~cm}$ in both $x$ and $y$ directions. . . . . . . 105

4.7 Probability (\%) for $10 \mathrm{~dB}$ outage. $L=1.6 \mathrm{Km} . \quad C_{n}^{2}=5 \times 10^{-15} \mathrm{~m}^{-2 / 3}$. Gaussian extra pointing error model. $\Delta x=\Delta y=2.5 \mathrm{~cm}$, and $\sigma_{b}=0.58 \mathrm{~cm}$, $\sigma_{p}=2.5 \mathrm{~cm}, \sigma_{d}=2.57 \mathrm{~cm}$ in both $x$ and $y$ directions. . . . . . . 106

4.8 Probability $(\%)$ for $10 \mathrm{~dB}$ outage. $L=4 \mathrm{Km} . C_{n}^{2}=5 \times 10^{-15} \mathrm{~m}^{-2 / 3}$. Beam wander only. $\Delta x=\Delta y=0 \mathrm{~cm}$, and $\sigma_{b}=2.29 \mathrm{~cm}, \sigma_{p}=0 \mathrm{~cm}, \sigma_{d}=2.29 \mathrm{~cm}$ in both $x$ and $y$ directions. . . . . . . . . . . . . . . 106

4.9 Probability $(\%)$ for $10 \mathrm{~dB}$ outage. $L=4 \mathrm{Km} . C_{n}^{2}=5 \times 10^{-15} \mathrm{~m}^{-2 / 3}$. Gaussian extra pointing error model. $\Delta x=\Delta y=0 \mathrm{~cm}$, and $\sigma_{b}=2.29 \mathrm{~cm}, \sigma_{p}=$ $1 \mathrm{~cm}, \sigma_{d}=2.5 \mathrm{~cm}$ in both $x$ and $y$ directions. . . . . . . . . . . 107 


\section{Acknowledgments}

I greatly thank my adviser, Professor Zhijun Zhao, who took me in as his graduate student and offered me the opportunity, guidance, and encouragement to finish my Ph.D. degree and this dissertation. I deeply appreciate his countless helpful insights, discussions, and advices throughout my Ph.D. study years. I am also grateful to Professors Daniel R. Fuhrmann, Haiying Liu, and Zhuo Feng, who served as my Ph.D. committee members and who provided invaluable help, discussing, comments, and feedback. 


\section{Abstract}

A free-space optical (FSO) laser communication system with perfect fast-tracking experiences random power fading due to atmospheric turbulence. For a FSO communication system without fast-tracking or with imperfect fast-tracking, the fading probability density function (pdf) is also affected by the pointing error. In this thesis, the overall fading pdfs of FSO communication system with pointing errors are calculated using an analytical method based on the fast-tracked on-axis and off-axis fading pdfs and the fast-tracked beam profile of a turbulence channel. The overall fading pdf is firstly studied for the FSO communication system with collimated laser beam. Large-scale numerical wave-optics simulations are performed to verify the analytically calculated fading pdf with collimated beam under various turbulence channels and pointing errors. The calculated overall fading pdfs are almost identical to the directly simulated fading pdfs. The calculated overall fading pdfs are also compared with the gamma-gamma $(\mathrm{GG})$ and the log-normal (LN) fading pdf models. They fit better than both the GG and LN fading pdf models under different receiver aperture sizes in all the studied cases. Further, the analytical method is expanded to the FSO communication system with beam diverging angle case. It is shown that the gamma pdf model is still valid for the fast-tracked on-axis and off-axis fading pdfs with point-like receiver aperture when the laser beam is propagated with beam diverging angle. Large-scale numerical wave-optics simulations prove that the analytically calculated fading pdfs perfectly fit the overall fading pdfs for both focused and diverged beam cases. The influence 
of the fast-tracked on-axis and off-axis fading pdfs, the fast-tracked beam profile, and the pointing error on the overall fading pdf is also discussed. At last, the analytical method is compared with the previous heuristic fading pdf models proposed since 1970s. Although some of previously proposed fading pdf models provide close fit to the experiment and simulation data, these close fits only exist under particular conditions. Only analytical method shows accurate fit to the directly simulated fading pdfs under different turbulence strength, propagation distances, receiver aperture sizes and pointing errors. 


\section{Chapter 1}

\section{Introduction}

This thesis is comprised of three related studies on the fading probability density function (pdf) of the free-space optical (FSO) communication system with pointing error. The first study, detailed in chapter 2, uses the analytical method to calculate the overall fading pdf of the FSO communication system with collimated laser beam and pointing error. The analytically calculated fading pdf shows perfect match to the directly simulated fading pdf under different turbulence strengths, propagation distances, receiver aperture sizes and pointing errors. Further, although the FSO communication system with collimated laser beam is the most studied case, study by Zhao, Liao and Zhang shows that better system performance can be achieved when the laser beam is transmitted with an optimal beam diverging angle [1]. The second study, detailed in chapter 3, expands the analytical method to the overall fading pdf of FSO communication system with beam diverging angle. The mathematical foundation is verified for the with beam diverging angle case. The analytically calculated fading pdfs perfectly fit the directly simulated fading pdfs. It is also found that when the laser beam is well focused, the overall fading pdf is affected by both on-axis and off-axis fast-tracked fading pdfs. When the laser beam is largely diverged, overall fading pdf can be approximated by the fast-tracked on-axis fading pdf. The third study, detailed in 
chapter 4, compares both the analytically calculated fading pdf and the previously proposed heuristic fading pdf models with the directly simulated fading pdf under different turbulence strengths, propagation distances, receiver aperture sizes and pointing errors. Both the turbulence induced beam wander only pointing error case and the more general pointing error case are considered. It is shown only the analytical method accurately estimates the fading pdfs in all the situations.

The rest of the this chapter is outlined as follows. Section 1.1 describes the motivation of studying the fading pdf of the FSO communication system. Section 1.2 reviews the study of the fading pdf models of the FSO communication system since 1970s. The mathematical forms of the previously proposed heuristic fading pdf models are given in section 1.3. Section 1.4 summarizes the key results found in each study. The outline of this thesis is given in section 1.5 .

\subsection{Motivation}

The free-space optical (FSO) laser communication system provides an attractive alternate to radio frequency (RF) systems due to the larger bandwidth, higher antenna gain, better privacy, smaller antenna and component sizes, and lower component costs [2]-[5]. They have been widely used in many applications, such as space communications, temporary network installations, safety add-ons for important fiber connections, aircraft-to-aircraft communications, the last-mile solutions, and military applications [6]-[10].

However, FSO communication also faces serious challenges [2], [11]-[20]. A perfectly fast-tracked FSO laser communication system experiences random power fading due to atmospheric turbulence [21]. For a FSO communication system without fast-tracking or with imperfect fast-tracking, the fading probability density function (pdf) is also affected by the 
pointing error. The pointing error is the overall displacement between the laser beam center and the receiver aperture center. The pointing error is generally composed of two components: the constant misalignment (also called boresight in the literature) and the random pointing error. Turbulence induced beam wander, optical misalignment, mechanical vibrations, and relative movements of the transmitter and the receiver are examples of sources of pointing errors. Previous study shows that the turbulence induced beam wander is equivalent to an independent two dimensional Gaussian random vibration [22]-[24]. When there is no tracking or only imperfect slow tracking, the pointing error consists of the overall effect of the turbulence induced beam wander and the relative mechanical displacement of the transmitter and receiver. When the relative movement of the transmitter and the receiver is eliminated by slow tracking, the pointing error only consists of the turbulence induced beam wander. With fast-tracking, the overall displacement caused by beam wander is also reduced. The residual fast-tracking error is then called pointing error.

In this thesis, an analytical method is used to calculate the overall fading pdfs for the FSO communication system through atmospheric turbulence channel with pointing error under different turbulence strength, propagation distances, receiver aperture sizes, beam diverging angles and pointing errors. The analytical method considers three factors, namely, the pointing error, the fast-tracked beam profile and the fast-tracked on-axis and off-axis fading pdfs [21], [25]-[28]. The overall channel fading pdf is formulated as two modulating independent random variables. One is the random fading of the fast-tracked laser beam, and the other is the random power fading induced by the pointing error on the fast-tracked beam profile. The exact pdfs are then obtained from direct large-scale numerical wave-optics simulations under different propagation parameters to verify the analytical formulation. The aerosol scattering effects [29], [30] caused by haze, rain, snow, and fog is not considered. 


\subsection{Background Review}

The system performance of the FSO communication system is essentially determined by the fading pdf of the received beam power. Since 1970s, different mathematical models are proposed to describe the fading pdf. In 1970s, the log-normal (LN) fading pdf is derived from the first order Rytov approximation. Although even the second order approximation of Rytov approximation shows it is not $\mathrm{LN}$, the model is widely accepted as the fading pdf model for the power fluctuation in the weak turbulence regime [11]. In 1979, the K distribution is regarded to fit well with the experiment data when the turbulence becomes relatively strong [31]. Later, the power fading is regarded to be better governed by doublely stochastic processes. In 1985, IK distribution is proposed as a universal model for the laser beam power fluctuation [32]. In 1987, the log-normal modulated exponential (LNME) and the Beckmann fading pdf models are proposed. The LNME fading pdf model shows good agreement with experiment data in strong turbulence regime [33]. The Beckmann fading pdf model is also called log-normal modulated Rician (LNMR) fading pdf model. In weak turbulence regime, the Beckmann fading pdf model reduces to $\mathrm{LN}$ fading pdf model. In strong turbulence regime, the Beckmann fading pdf model reduces to LNME fading pdf model [34], [35]. In 1989, the IK fading pdf model and the Beckmann fading pdf model are compared with the measured experiment data. The comparison shows the Beckmann fading pdf model fits better with the experiment data [36]. In 2001, gamma-gamma (GG) fading pdf model is proposed [37]. The GG fading pdf model is regarded to provide a good fit to the fading pdf when the receiver aperture is much smaller than the coherence radius in the moderate-to-strong turbulence regime [38]. The parameters of the GG fading pdf model are supposed to be directly calculated from atmospheric parameters. However, later study shows that GG fading pdf model with parameters directly calculated from atmospheric parameters does not match well with the simulated fading pdf. The parameters of the GG fading pdf model are obtained by doing a best fit to the simulated fading pdf [38]. 
In the past, the previously proposed heuristic fading pdf models are verified in some extents by theoretical analysis or physical experiment. However, due to the limit of theoretical analysis and physical experiment, the verification is not sufficient. In the early time, the mathematical models are verified by comparing the moments of the proposed model with the moments from the observed data of the laser power fluctuation [31], [32], [39]. The comparison of the moments is inadequate to verify the fading pdf models as the fading pdf contains the moment information of all the orders and only lower order moments were compared. The fading pdf models are later compared with the fading pdf obtained from the physical experiment. However, due to the difficulties in physical experiment, fading pdf is obtained with limited precision, especially at the deep fading tail which is critical in determining the system BER performance. The numerical simulations are developed based on the turbulence theory in the 1990s. The numerical simulation is proved to be an approach that can accurate describe the FSO communication system power fading [40], [41]. The numerical simulation is actually recommended in studying the fading pdf [35]. The problem for the numerical simulation is that it has a high computational complexity. To accurately simulate the turbulence channel, a hug amount of computation is required. In this thesis, the numerical simulation is adopted as the approach to study fading pdf of the FSO communication system. The numerical simulation is pushed to a much higher precision than those in the previously published papers to accurately simulate the laser beam propagating in the turbulence channel.

It is also needed to be noted that, although some of the fading pdf models show agreement to the experiment or simulated fading pdf, the agreement only exists under certain special conditions. For instance, the LN fading pdf model is regarded to be a good approximation in weak turbulence regime. Later, it is regarded to fit well with fading pdf in moderate-tostrong turbulence regime when the receiver aperture size is much larger than the coherence radius [38]. The GG fading pdf model is regarded to provide good fit in moderate-to-strong turbulence regime when the receiver aperture size is much smaller than the coherence radius [38]. 


\subsection{Mathematical Fading Pdf Models Review}

The performance of the FSO communication system is greatly determined by the random fluctuations of the received laser beam power. The power fluctuation is also called fading. For four decades, people are working to find a mathematical model that accurately describes the power fading pdf of the FSO communication system, especially for the deep

fading tail. A number of heuristic fading pdf models have been proposed and better fits to the experiment and simulation data under certain conditions are observed. These mathematical models include models containing single density function, such as LN fading pdf model, and doubly stochastic models such as LNME, Beckmann, and GG fading pdf models. The doubly stochastic model assumes the power fluctuations arise from two multiplicative random processes [11], [33], [34], [37], [38].

\subsubsection{Log-normal (LN) Fading Pdf Model}

The LN fading pdf model is derived from the first order Rytov approximation, where the logarithm of the power fluctuation can be approximated by the Gaussian distribution. A $\mathrm{LN}$ fading pdf model with mean $\mathrm{E}[I]=1$ is described as [38]

$$
p_{I}(I)=\frac{1}{I \sqrt{2 \pi \sigma_{\ln I}^{2}}} \exp \left[-\frac{\left[\ln (I)+\frac{1}{2} \sigma_{\ln I}^{2}\right]^{2}}{2 \sigma_{\ln I}^{2}}\right], \quad I>0
$$

where $\sigma_{\ln I}^{2}$ is the variance of the normalized beam power in log scale, where

$$
\sigma_{\ln I}^{2}=\ln \left(\sigma_{I}^{2}+1\right)
$$


where $\ln$ is the natural logarithm. In the weak turbulence is $\sigma_{\ln I}^{2}$ approximately equal to $\sigma_{I}^{2}$ as

$$
\begin{aligned}
\sigma_{\ln I}^{2} & =\ln \left(\sigma_{I}^{2}+1\right) \\
& \approx \sigma_{I}^{2}
\end{aligned}
$$

Further, for spherical wave [42],

$$
\sigma_{I}^{2}=\exp \left[\frac{0.49 \sigma_{R}^{2}}{\left(1+0.18 d^{2}+0.56 \sigma_{R}^{12 / 5}\right)^{7 / 6}}+\frac{0.51 \sigma_{R}^{2}}{\left(1+0.9 d^{2}+0.62 d^{2} \sigma_{R}^{12 / 5}\right)^{5 / 6}}\right]-1
$$

where $D$ is the receiver aperture diameter, $\lambda$ is the optical wavelength,

$$
d=\sqrt{k D^{2} / 4 L}
$$

wavenumber $k$ is

$$
k=2 \pi / \lambda
$$

Rytov variance $\sigma_{R}^{2}$ is

$$
\sigma_{R}^{2}=1.23 C_{n}^{2} k^{7 / 6} L^{11 / 6}
$$

where $C_{n}^{2}$ is the strength of the atmospheric turbulence. 


\subsubsection{K Fading Pdf Model}

$\mathrm{K}$ distribution is proposed for the fading pdf with a relatively strong turbulence [31] in 1979. With the normalized received beam power $I$,

$$
P(I)=\frac{2}{\Gamma(\alpha)} \alpha^{(\alpha+1) / 2} I^{(\alpha-1) / 2} K_{\alpha-1}\left[2(I \alpha)^{1 / 2}\right]
$$

where $\alpha=2 /\left(\mathrm{E}\left[I^{2}\right]-2\right), K_{v}(z)$ is the modified Bessel function of the second kind, and $\Gamma(y)$ is the gamma function.

Later, the $\mathrm{K}$ distribution is shown to be able to be derived from the doubly stochastic theory. The doubly stochastic theory assumes the fading pdf is the result of the modulation of two scholastic processes [12]. In the $\mathrm{K}$ distribution [37], the fading pdf can be regarded to be governed by the conditional exponential distribution $p_{1}(I \mid b)$ where

$$
p_{1}(I \mid b)=\frac{1}{b} \exp (-I / b), I>0
$$

with mean value $b$ follow the gamma distribution where

$$
p_{2}(b)=\frac{\alpha(\alpha b)^{\alpha-1}}{\Gamma(\alpha)} \exp (-\alpha b), b>0, \alpha>0
$$

Hence

$$
p(I)=\int_{0}^{\infty} p_{1}(I \mid b) p_{2}(b) d b
$$




\subsubsection{IK Fading Pdf Model}

As both LN and K fading pdf models only fit the moments of the experiment data under certain turbulence strength conditions, the IK distribution is proposed as a universal fading pdf model in 1985 [39]. IK fading pdf model is a doubly stochastic model, which assumes that the received laser power fluctuations are induced by the modulation of conditional distribution $p_{1}(I \mid b)$ with the mean value $b$ which follows the distribution $p_{2}(b)$. IK fading pdf model assumes conditional distribution $p_{1}(I \mid b)$ follows the $n$ distribution where

$$
p_{1}(I \mid b)=\frac{\alpha}{b}\left(\frac{\sqrt{I}}{A}\right)^{\alpha-1} \exp \left[-\alpha\left(A^{2}+I\right) / b\right] I_{\alpha-1}\left(\frac{2 \alpha A}{b} \sqrt{I}\right), I>0
$$

and the mean value $b$ follow the exponential distribution where

$$
p_{2}(b)=\frac{1}{b_{0}} \exp \left(-b / b_{0}\right), b>0
$$

where $b_{0}$ is the mean value of $b$ [39].

Hence

$$
p(I)=\int_{0}^{\infty} p_{1}(I \mid b) p_{2}(b) d b
$$

and

$$
p(I)=\left\{\begin{array}{l}
\frac{2 \alpha}{b_{0}}\left(\frac{\sqrt{I}}{A}\right)^{\alpha-1} K_{\alpha-1}\left(2 A \sqrt{\frac{\alpha}{b_{0}}}\right) I_{\alpha-1}\left(2 \sqrt{\frac{\alpha I}{b_{0}}}\right), I<A^{2} \\
\frac{2 \alpha}{b_{0}}\left(\frac{\sqrt{I}}{A}\right)^{\alpha-1} I_{\alpha-1}\left(2 A \sqrt{\frac{\alpha}{b_{0}}}\right) K_{\alpha-1}\left(2 \sqrt{\frac{\alpha I}{b_{0}}}\right), I>A^{2}
\end{array}\right.
$$

where $I_{v}(z)$ is the modified Bessel function of the first kind, $K_{v}(z)$ is the modified Bessel 
function of the second kind. The moment of the IK distribution [39] is

$$
\mathrm{E}\left[I^{n}\right]=\left(\frac{b_{0}}{\alpha}\right)^{n} n ! \sum_{k=0}^{n} \frac{\Gamma(\alpha+n)}{\Gamma(\alpha+k)} \frac{\alpha^{k} \rho^{k}}{k !}
$$

where $\Gamma$ is the gamma function, and

$$
\rho=A^{2} / b_{0}
$$

As the $I$ is normalized to unity,

$$
b_{0}=\frac{1}{1+\rho}
$$

Hence 1.16 can be written as

$$
p(I)=\left\{\begin{array}{l}
2 \alpha(1+\rho)\left[\frac{(1+\rho) I}{\rho}\right]^{(\alpha-1) / 2} K_{\alpha-1}\left[2(\alpha \rho)^{1 / 2}\right] I_{\alpha-1}\left\{2[\alpha(1+\rho) I]^{1 / 2}\right\}, I<\frac{\rho}{1+\rho} \\
2 \alpha(1+\rho)\left[\frac{(1+\rho) I}{\rho}\right]^{(\alpha-1) / 2} I_{\alpha-1}\left[2(\alpha \rho)^{1 / 2}\right] K_{\alpha-1}\left\{2[\alpha(1+\rho) I]^{1 / 2}\right\}, I>\frac{\rho}{1+\rho}
\end{array}\right.
$$

where $\alpha$ is the effective number of scatterers, $\rho$ is the coherence parameter, $I_{v}(z)$ is the modified Bessel function of the first kind and $K_{v}(z)$ is the modified Bessel function of the second kind [36], [39]. The second and third moments can be calculated by

$$
\begin{aligned}
& \mathrm{E}\left[I^{2}\right]=\frac{\rho^{2}}{(1+\rho)^{2}}+2 \frac{1+\alpha^{-1}}{1+\rho} \\
& \mathrm{E}\left[I^{3}\right]=\frac{\rho^{3}}{(1+\rho)^{3}}+3 \frac{\left(1+2 \alpha^{-1}\right) \rho^{2}}{(1+\rho)^{3}}+6 \frac{\left(1+\alpha^{-1}\right)\left(1+2 \alpha^{-1}\right)}{(1+\rho)^{2}}
\end{aligned}
$$


and the parameter $\rho$ can be solved from the cubic equation [36]

$$
\begin{aligned}
& \left(\mathrm{E}\left[I^{2}\right]^{2}-\mathrm{E}\left[I^{2}\right]-\frac{1}{3} \mathrm{E}\left[I^{3}\right]+\frac{1}{3}\right) \rho^{3}+\left(3 \mathrm{E}\left[I^{2}\right]^{2}-2 \mathrm{E}\left[I^{2}\right]-\mathrm{E}\left[I^{3}\right]\right)\left(\rho^{2}+\rho\right)+ \\
& \mathrm{E}\left[I^{2}\right]^{2}-\mathrm{E}\left[I^{2}\right]-\frac{1}{3} \mathrm{E}\left[I^{3}\right]=0
\end{aligned}
$$

and then $\alpha$ can be solved as

$$
\alpha^{-1}=\frac{1}{2}(1+\rho) \mathrm{E}\left[I^{2}\right]-\frac{1}{2} \frac{\rho^{2}}{1+\rho}-1
$$

The limit of the IK fading pdf model in weak turbulence regime is induced by letting $\rho$ become large and letting $\alpha$ become small in equation 1.20 [36]. Hence,

$$
p(I)=\frac{1}{\sqrt{2} \sigma} I^{-3 / 4} \exp \left(-\frac{2^{3 / 2}}{\sigma}|1-\sqrt{I}|\right), \sigma \ll 1
$$

where $\sigma^{2}$ is the standard deviation of the IK fading pdf and $\sigma^{2}=2 /(\alpha \rho)$ in this weak turbulence regime.

In strong turbulence regime, the coherence parameter $\rho$ goes to zero, and the IK fading pdf model reduces to the $\mathrm{K}$ fading distribution model [36].

\subsubsection{LNME Fading Pdf Model}

The log-normally modulated exponential (LNME) fading pdf model is a doubly stochastic model. In the LNME fading pdf model, $p_{1}(I \mid b)$ is assumed to follow the exponential 
distribution and $p_{2}(b)$ is assumed to follow the LN distribution [33]. As

$$
\begin{gathered}
p(I)=\int_{0}^{\infty} p_{1}(I \mid b) p_{2}(b) d b \\
p(I)=\frac{1}{\sqrt{2 \pi} \sigma_{b}} \int_{0}^{\infty} \frac{d b}{b^{2}} \exp \left[-\frac{I}{b}-\frac{\left(\ln b+\frac{1}{2} \sigma_{b}^{2}\right)^{2}}{2 \sigma_{b}^{2}}\right]
\end{gathered}
$$

where $\sigma_{b}^{2}$ is the variance of the logarithm of the modulation [33], where

$$
\sigma_{b}^{2}=\ln \left(\frac{\sigma_{I}^{2}+1}{2}\right)
$$

where $\sigma_{I}^{2}$ is the variance of the normalized received laser beam power.

\subsubsection{Beckmann (LNMR) Fading Pdf Model}

Beckmann fading pdf model is also called log-normally modulated Rician (LNMR) fading pdf model. The Beckmann fading pdf model is also a doubly stochastic model. It assumes the fading pdf is the modulation of the Rice-Nakagami distribution and the LN distribution [34], [43].

The Rice-Nakagami distribution is

$$
P_{R N}(I \mid b, r)=(r+1) b^{-1} \exp \left[-r-(r+1) \frac{I}{b}\right] I_{0}\left\{\left[4 r(r+1) \frac{I}{b}\right]^{1 / 2}\right\}
$$

where $I$ is the received beam power, $b$ is the mean value, and $I_{v}(z)$ is the modified Bessel function of the first kind, $r$ is the coherence parameter. In weak turbulence regime $r$ grows to infinity and in strong turbulence regime $r$ reduces to 0 .

The LN distribution is 


$$
P_{L N}(b)=\frac{1}{\sqrt{2 \pi} \sigma_{b} b} \exp \left[-\frac{\left(\ln b+\frac{1}{2} \sigma_{b}^{2}\right)^{2}}{2 \sigma_{b}^{2}}\right]
$$

where $\sigma_{b}^{2}$ is the variance of $\ln b$ and the mean of $b$ is unity. Then, the Beckmann fading pdf model is given by

$$
P_{B}\left(I \mid r, \sigma_{b}^{2}\right)=\int_{0}^{\infty} d b P_{R N}(I \mid b, r) P_{L N}\left(b \mid \sigma_{b}^{2}\right)
$$

For weak turbulence regime, the Beckmann fading pdf reduces to the log-normal fading pdf; For strong turbulence regime, the Beckmann fading pdf reduces to the LNME fading pdf [35].

The coherence parameter $r$ and the variance of the $\mathrm{LN}$ distribution $\sigma_{b}^{2}$ are required to generate the Beckmann fading pdf. The parameters can be obtained from the moments of the physical or numerical experimental data [35] as

$$
\begin{aligned}
\mathrm{E}\left[I^{2}\right] & =\exp \left(\sigma_{b}^{2}\right)\left(r^{2}+4 r+2\right) /(r+1)^{2} \\
\mathrm{E}\left[I^{-1 / 2}\right] & =\pi^{1 / 2}(r+1)^{1 / 2} \exp (-r / 2) I_{0}(r / 2) \exp \left(3 \sigma_{b}^{2} / 8\right) \\
\exp (\mathrm{E}[\ln I]) & =[r /(r+1)] \exp \left[-\frac{1}{2} \sigma_{b}^{2}+E_{1}(r)\right]
\end{aligned}
$$

where

$$
E_{1}(r)=\int_{r}^{\infty} d t t^{-1} \exp (-t)
$$

Any of the two above three equations can be used to calculate the unknowns $r$ and $\sigma_{b}^{2}$. However, a solution can not be obtained in some cases [44]. 


\subsubsection{Gamma-Gamma (GG) Fading Pdf Model}

In the Gamma-Gamma (GG) fading pdf model, the fading pdf is modeled as the modulation of two statistically independent Gamma distributed process $p_{1}(x)$ and $p_{2}(y)$ where $I=$ xy [37], [42]. Hence

$$
p(I)=\frac{2}{\Gamma(\alpha) \Gamma(\beta) I}(\alpha \beta I)^{(\alpha+\beta) / 2} K_{\alpha-\beta}(2 \sqrt{\alpha \beta I}), \quad I>0
$$

where $I$ is the normalized received laser beam power, $\Gamma(x)$ is the gamma function, and $K_{v}(z)$ is the modified Bessel function of the second kind.

In the GG fading pdf model, parameters $\alpha$ and $\beta$ are regarded to represent the effective numbers of large-scale and small-scale scatterers of the turbulence channel [12], [37], [42], where

$$
\mathrm{E}\left[I^{2}\right]=\left(1+\frac{1}{\alpha}\right)\left(1+\frac{1}{\beta}\right)
$$

As $I=x y$

$$
\mathrm{E}\left[I^{2}\right]=\mathrm{E}\left[x^{2}\right] \mathrm{E}\left[y^{2}\right]=\left(1+\sigma_{x}^{2}\right)\left(1+\sigma_{y}^{2}\right)
$$

where $\sigma_{x}^{2}$ and $\sigma_{y}^{2}$ are the normalized variance of the large-scale and small-scale scatterers, respectively. Hence, 


$$
\begin{aligned}
& \alpha=\frac{1}{\sigma_{x}^{2}}=\frac{1}{\exp \left(\sigma_{\ln x}^{2}\right)-1} \\
& \beta=\frac{1}{\sigma_{y}^{2}}=\frac{1}{\exp \left(\sigma_{\ln y}^{2}\right)-1}
\end{aligned}
$$

And $\sigma_{I}^{2}$ can be calculated by $\sigma_{\ln x}^{2}$ and $\sigma_{\ln y}^{2}$ as

$$
\sigma_{I}^{2}=\exp \left(\sigma_{\ln x}^{2}+\sigma_{\ln y}^{2}\right)-1
$$

\subsection{Summary of Key Results}

This thesis studies the overall fading pdf of the received beam power of the FSO communication system with pointing error. In the study detailed in chapter 2 , an analytical method is used to calculate the overall fading pdf for the FSO communication system with collimated laser beam. In the analytical method, the overall channel fading pdf is formulated as two modulating independent random variables. One is the random fading of the fast-tracked laser beam, and the other is the random power fading induced by the pointing error on the fast-tracked beam profile. The pointing error is the overall displacement between the laser beam weight center and the receiver aperture center at the receiver plane. Given a FSO channel, the fast-tracked beam profile and the fast-tracked on-axis and off-axis fading pdfs are obtained by numerical simulations. With these channel information, the overall fading pdf is directly calculated using the analytical method with the pointing error model. Large scale direct wave-optics numerical simulations with collimated Gaussian beam along a horizontal direction are performed to verify the analytically calculated fading pdfs. The simulations are performed with the high resolution that is sufficient to study the turbulence 
channel. The FSO channels with different propagation distances, turbulence strength and receiver aperture sizes are studied. The laser wavelength is $\lambda=1.54 \times 10^{-6} \mathrm{~m}$. The refractive index structure parameter ranges from $5 \times 10^{-15} \mathrm{~m}^{-2 / 3}$ to $1 \times 10^{-13} \mathrm{~m}^{-2 / 3}$. The propagation distance ranges from $0.4 \mathrm{Km}$ to $4 \mathrm{Km}$. The Rytov variance ranges from 0.24 to 1.27. The simulations cover from weak turbulence regime to strong turbulence regime. The coherence radius ranges from $1.6 \mathrm{~cm}$ to $2.4 \mathrm{~cm}$. The receiver aperture size ranges from point-like $0 \mathrm{~cm}$ aperture to aperture with $20 \mathrm{~cm}$ diameter. To accurately obtain especially the deep fading tail of the fading pdf, the simulations are conducted under large grid size with high resolution. The field grid size ranges from $1024 \times 1024$ to $2048 \times 2048$. The field resolution ranges from $0.625 \mathrm{~mm}$ to $2.5 \mathrm{~mm}$, which is much smaller than the coherence radius in each case. The iteration number ranges from 0.4 million to 1 million. Both the fast-tracking residual error model and the beam wander plus transceiver induced extra pointing error model are studied. Three transceiver induced extra pointing error models are studied. They are the Gaussian random model, the uniform random model, and the sine sway model. The analytical method accurately estimates the fading pdf in all our studied cases. The analytical method is also compared with both the wildly accepted GG and LN fading pdf models. The GG fading pdf model is regarded to be a good estimation of the overall fading pdf if the receiver aperture size is much smaller than the coherence radius. And the LN model is regarded to fit the fading pdf if the receiver aperture size is much larger than the receiver aperture size [38]. However, the analytical method shows a better fit than both the GG and LN fading pdf models from point-like receiver aperture which is much smaller than the coherence radius to the $20 \mathrm{~cm}$ receiver aperture which is much larger than the coherence radius in our studied cases. The analytical method accurately estimates the fading pdfs in all the studied pointing errors. Both the GG model and the LN model underestimate the average BER of the FSO communication system. The outage probability is also underestimated by both the GG and LN fading pdf models. Due to the long deep fading period of the slow fading channel, this will largely underestimate the system performance degradation caused by outage to the system. 
Although the FSO communication system with collimated laser beam is the most studied case, Zhao, Liao and Zhang show that the FSO communication system can achieve better system performance with an optimal beam diverging angle [1]. The study detailed in chapter 3 expands the analytical method to the FSO communication system with beam diverging angle case. The pointing error is the turbulence induced beam wander. Large-scale numerical simulations show that for Gaussian laser beam transmitted through the atmospheric turbulence channel with ether a focused or diverged beam angle, the fast-tracked on-axis and off-axis fading pdfs obtained with point-like receiver can also be well modeled by the gamma pdf model.

When the laser beam is focused, the fast-tracked beam profile size is small. And the fast-tracked fading pdf changes rapidly as the receiver aperture moves apart from the fasttracked beam center. When the beam diverging angle increases, the fast-tracked beam spot size grows larger, the fast-tracked fading pdf changes much slower compared to the focused beam case. The size of the fast-tracked beam profile is close to the diffraction pattern in both focused and diverged beam cases. It is a little larger due to the turbulence induced beam broadening effect. For the turbulence induced beam wander, it is shown that the standard deviation of the two dimensional Gaussian distributed beam wander slightly decreases as the beam diverging angle increases. Hence for the focused beam, as the fast-tracked fading pdf changes fast and the beam wander range, which is defined as the three times of the beam wander standard deviation, is large, it is likely that the fast-tracked fading pdf keeps changing in the beam wander range. Both on-axis and off-axis fast-tracked fading pdfs in the beam wander range contribute to the overall fading pdf. When the beam diverging angle grows much larger, as the fast-tracked beam profile and the fast-tracked fading pdf change much slower and the beam wander range decreases, the analytical method can be well approximated by the fast-tracked on-axis fading pdf. In this case, for the point-like receiver aperture, a single gamma pdf model is adequate to represent the overall fading pdf given the variance of the normalized received laser beam power. 
The study detailed in chapter 4 compares the analytical method and the previously proposed heuristic fading models with the directly simulated fading pdf. Only the analytical method accurately fits the directly simulated fading pdf under different turbulence strengths, propagation distances, receiver aperture sizes and pointing errors. When there is only turbulence induced beam wander pointing error, and with point-like receiver aperture, Beckmann fading pdf model can well estimate the overall fading pdfs. However, Beckmann fading pdf model fails otherwise in our study. Both the analytical method and Beckmann fading pdf model require certain amount of information from the channel fading. For the analytical method, given the fast-tracked on-axis and off-axis fading pdfs, the fast-tracked beam profile and the pointing error, the overall fading pdfs can be directly calculated. For the Beckmann model, the $-1 / 2$ order and the second order moments are required to calculated the parameters of the Beckmann model. However, a solution is not always guaranteed for the Beckmann fading pdf model parameters. With turbulence induced beam wander only pointing error and point-like receiver, the GG fading pdf model provides good fit when the beam wander effect is weak. The GG fading pdf model deviates from the deep fading tail when the beam wander effect becomes strong. The GG fading pdf model is different from the simulated fading pdf under larger receiver aperture or under more general pointing error. Although the parameters of the GG fading pdf model are connected to the atmospheric turbulence parameters, to better fit the simulated fading pdf, the parameters are currently obtained from the best fit of the simulated fading pdf. The parameters of both the LN fading pdf model and the approximation of the IK distribution in weak turbulence condition (IK Weak) can be directly obtained from the normalized overall fading variance. However the LN fading pdf model and IK Weak fading pdf model provide the least accurate fit compared with all the fading pdf models mentioned above. When the turbulence induced beam wander is partially compensated by the fast-tracking system, or when the mechanical induced extra pointing error is involved, or when the receiver aperture grows larger, only the analytically calculated fading pdf can provide accurate match to the direct simulated fading pdf. 


\subsection{Organization of Dissertation}

The remainder of this thesis is organized as follows. In chapter 2, an analytical method is used to calculate the overall fading pdf of the FSO communication system with collimated laser beam and pointing error. In chapter 3, the analytical method is expanded to calculate the overall fading pdf of the FSO communication system with laser beam propagated with diverging angle. In chapter 4, the analytical method is compared with the previously proposed heuristic fading pdf models. Conclusions are given in chapter 5. 


\section{Chapter 2}

\section{Fading Pdf for FSO Communication System with Pointing Error}

\subsection{Introduction}

The free-space optical (FSO) laser communication system provides an attractive alternate to radio frequency (RF) systems due to the larger bandwidth, higher antenna gain, better privacy, smaller antenna and component sizes, and lower component costs [2], [3]. They have been widely used in many applications, such as space communications, temporary network installations, safety add-ons for important fiber connections, aircraft-to-aircraft communications, the last-mile solutions, and military applications [6]-[10].

However, FSO communication also faces serious challenges. A perfectly fast-tracked FSO laser communication system experiences random power fading due to atmospheric turbulence [21]. For a FSO communication system without fast-tracking or with imperfect fast-tracking, the fading probability density function (pdf) is also affected by the pointing 
error [27], [28], [45]-[52]. The pointing error is the overall displacement between the laser beam center and the receiver aperture center. The pointing error is generally composed of two components: the constant misalignment (also called boresight in the literature) and the random pointing error. Turbulence induced beam wander, optical misalignment, mechanical vibrations, and relative movements of the transmitter and the receiver are examples of sources of pointing errors [26], [53]. Previous study shows that the turbulence induced beam wander is equivalent to an independent two dimensional Gaussian random vibration [22]-[24]. When there is no tracking or only imperfect slow tracking, the pointing error consists of the overall effect of the turbulence induced beam wander and the relative mechanical displacement of the transmitter and receiver. When the relative movement of the transmitter and the receiver is eliminated by slow tracking, the pointing error only consists of the turbulence induced beam wander. The pointing error is called the beam wander plus extra pointing error. With fast-tracking, the overall displacement caused by beam wander is also reduced. The residual fast-tracking error is then called pointing error.

In this chapter, an analytical method is used to calculate the overall fading pdfs for the FSO communication system with collimated laser beam propagating through atmospheric turbulence channel with pointing error. The exact pdfs are then obtained from direct largescale numerical wave-optics simulations under different propagation parameters to verify the analytical formulation. The aerosol scattering effects [29], [30] caused by haze, rain, snow, and fog is not considered.

The analytical method considers three factors, namely, the pointing error, the fast-tracked beam profile and the fast-tracked on-axis and off-axis fading pdfs [21], [22], [25], [26]. The overall channel fading pdf is formulated as two modulating independent random variables. One is the random fading of the fast-tracked laser beam, and the other is the random power fading induced by the pointing error on the fast-tracked beam profile.

Zhao and Liao showed that when the receiver aperture size is smaller than the coherence 
radius, both on-axis and off-axis fading pdfs of the fast-tracked laser beam can be closely modeled by the gamma pdfs. Numerical simulation is adopted to provide the fast-tracked beam profile and fast-tracked on-axis and off-axis fading pdfs information. For each turbulence channel, once the above information is obtained, the overall fading pdf with pointing error is directly calculated using the analytical method.

In this chapter, the FSO system uses the collimated Gaussian laser beam along a horizontal direction. The laser wavelength is $\lambda=1.54 \times 10^{-6} \mathrm{~m}$. The simulations are performed under different turbulence strengths and propagation distances. The refractive index structure parameter ranges from $5 \times 10^{-15} \mathrm{~m}^{-2 / 3}$ to $1 \times 10^{-13} \mathrm{~m}^{-2 / 3}$. The propagation distance ranges from $0.4 \mathrm{Km}$ to $4 \mathrm{Km}$. The receiver aperture size ranges from point-like $0 \mathrm{~cm}$ aperture to aperture with $20 \mathrm{~cm}$ diameter. The simulations cover from weak to strong fluctuation regime. To accurately obtain especially the deep fading tail of the fading pdf, the simulations are conducted under large grid size with high resolution. The field grid size ranges from $1024 \times 1024$ to $2048 \times 2048$. The field resolution ranges from $0.625 \mathrm{~mm}$ to $2.5 \mathrm{~mm}$, which is much smaller than the coherence radius in each case. The iteration number ranges from 0.4 million to 1 million.

The overall fading pdfs calculated using the analytical method accurately match the corresponding fading pdfs obtained from numerical wave-optics simulations in all the studied cases. They also fit better than the best fitted gamma-gamma (GG) and log-normal (LN) fading pdf models under different aperture sizes in all the studied cases. The differences are then examined in system bit error rate (BER) and the outage probability.

The rest of this chapter is organized as follows. Section 2.2 derives the analytical method to calculate the overall fading pdf of the FSO channel. Section 2.3 gives the mathematical expression of some common pointing errors. Section 2.4 describes the direct wave-optics simulation setup. The calculated overall fading pdf and the direct simulated fading pdf are compared in section 2.5. Conclusions are given in section 2.6. 


\subsection{Analytical Method}

For a FSO laser communication system without fast-tracking or with imperfect fast-tracking, the laser beam moves at the receiver plane due to the pointing error. The pointing error is the overall displacement between the beam weight center and the receiver aperture center. Denote $x_{d}, y_{d}$ as the overall displacement in $x$ and $y$ directions, $U_{x_{d}, y_{d}}(x, y)$ as the average beam profile of the random laser beams with beam center at location $\left(x_{d}, y_{d}\right)$, where $(x, y)$ is the displacement from the beam center at $x$ and $y$ directions. In our study, the fast-tracked beam profile $U_{x_{d}, y_{d}}(x, y)$ can be regarded to be independent with beam center location $\left(x_{d}, y_{d}\right)$ and follow the same form as the fast-tracked beam profile $U(x, y)$. The normalized fast-tracked on-axis and off-axis fading pdfs are also assumed to be independent with the beam center location $\left(x_{d}, y_{d}\right)$ and follow the same set of fast-tracked fading pdfs. Assuming the source beam is two dimensional symmetrical and the atmospheric turbulence is homogeneous in both horizontal and vertical directions, the fast-tracked beam profile is two dimensional symmetrical at the receiver plane. Denote $\rho$ as

$$
\rho^{2}=x_{d}^{2}+y_{d}^{2}
$$

with the same receiver aperture, the displacement $\rho$ and the average received power $I_{\text {displace }}$ at $\rho$ are bijective, where

$$
I_{\text {displace }}=f(\rho)
$$

As the fast-tracked beam profile follows the form $U(x, y)$, the average received power $I_{\text {displace }}$ when the displacement is $\rho$ with receiver aperture area $A$ is 


$$
I_{\text {displace }}=\iint_{A(\rho)} U(x, y) d x d y
$$

Denote $P_{d}\left(I_{\text {displace }}\right)$ as the pdf of receiving average power $I_{\text {displace }}$ caused by the pointing error based on the fast-tracked beam spot, as $\rho$ and $I_{\text {displace }}$ are bijective, the cumulative distribution function (cdf) of $I_{\text {displace }}$ is

$$
F_{I_{\text {displace }}}\left(I_{\text {displace }}\right)=\int_{0}^{f^{-1}\left(I_{\text {displace }}\right)} P(\rho) d \rho
$$

By differentiating 2.4 with respect to $I_{\text {displace }}$,

$$
P_{d}\left(I_{\text {displace }}\right)=\frac{d \rho}{d I_{\text {displace }}} P(\rho)
$$

And it can be further written as

$$
P_{d}\left(I_{\text {displace }}\right)=\frac{d \rho^{2}}{d I_{\text {displace }}} P\left(\rho^{2}\right)
$$

As the over probability of receiving instantaneous beam power $I_{\text {overall }}$ can be written as

$$
P_{\text {overall }}\left(I_{\text {overall }}\right)=\int_{0}^{\infty} P\left(I_{\text {overall }} \mid I_{\text {displace }}\right) P_{d}\left(I_{\text {displace }}\right) d I_{\text {displace }}
$$

as

$$
\begin{gathered}
P\left(I_{\text {overall }} \mid I_{\text {displace }}\right) d I_{\text {overall }}=P_{t}\left(\frac{I_{\text {overall }}}{I_{\text {displace }}} \mid I_{\text {displace }}\right) d\left(\frac{I_{\text {overall }}}{I_{\text {displace }}}\right) \\
P\left(I_{\text {overall }} \mid I_{\text {displace }}\right)=P_{t}\left(\frac{I_{\text {overall }}}{I_{\text {displace }}} \mid I_{\text {displace }}\right) / I_{\text {displace }}
\end{gathered}
$$


Hence

$$
P_{\text {overall }}\left(I_{\text {overall }}\right)=\int_{0}^{\infty} P_{t}\left(\frac{I_{\text {overall }}}{I_{\text {displace }}} \mid I_{\text {displace }}\right) \frac{P_{d}\left(I_{\text {displace }}\right)}{I_{\text {displace }}} d I_{\text {displace }}
$$

where $P_{t}\left(\frac{I_{\text {overall }}}{I_{\text {displace }}} \mid I_{\text {displace }}\right)$ is the normalized fast-tracked fading pdf at on-axis and off-axis locations.

\subsection{Pointing Error Models}

Denote the position of the receiver aperture center as $(0,0)$. The pointing error is the overall random movement of the beam weight center $\left(x_{d}, y_{d}\right)$ [21], [47]. As shown in figure 2.1, the solid cycle represents the receiver aperture with center at $(0,0)$. The dash cycle represents the average beam spot of the received random beams with beam center at $\left(x_{d}, y_{d}\right)$. The pointing error of the optical beam can be caused by either fast-tracking residual, turbulence induced beam wander, or the overall effects of beam wander and transceiver induced extra pointing error. Fast-tracking residual error is caused by imperfect fast-tracking. Beam wander only pointing error is the result of the perfect slow-tracking but absence of fasttracking. Beam wander plus transceiver induced extra pointing error is caused by ether imperfect slow-tracking or the absence of slow-tracking. The beam wander only model can be regarded to be ether a special case of the fast-tracking residual case where $\Delta x=\Delta y=0$, $\sigma_{p}=\sigma_{b}$, or a special case of the beam wander plus transceiver displacement case where the transceiver displacement is a pulse with no misalignment. Hence only the fast-tracking residual model and the beam wander plus transceiver induced extra pointing error model are studied in this chapter.

In the fast-tracking residual error model, the transceiver induced extra pointing error is 
supposed to be eliminated by the tracking system. The turbulence induced Gaussian beam wander is also reduced. Only a small amount of random displacement remains as the fast-tracking residual. The residual is assumed to follow the two dimensional Gaussian distribution in this chapter. In the beam wander plus transceiver induced extra pointing error model, the random displacement is consisted with both the turbulence induced random beam wander and the transceiver induced extra pointing error. The probability distribution of the transceiver induced extra pointing error is studied by the Gaussian random model, the uniform random model, and the sine sway model.

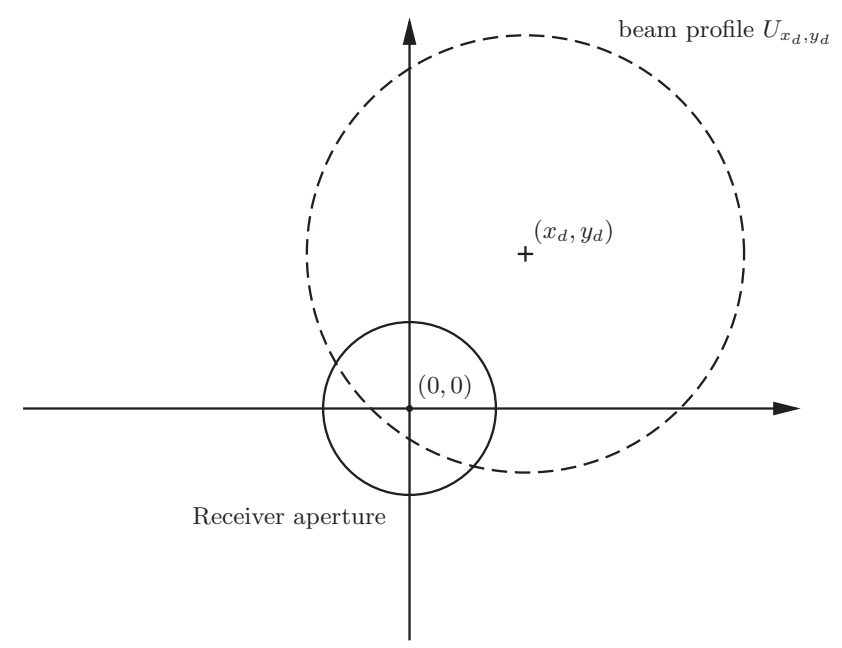

Figure 2.1: Random walk of the beam center.

\subsubsection{Fast-tracking Residual Error}

The fast-tracking residual is assumed to follow two dimensional Gaussian distribution where

$$
\begin{aligned}
& x_{d} \sim N\left(\Delta x, \sigma_{d x}^{2}\right) \\
& y_{d} \sim N\left(\Delta y, \sigma_{d y}^{2}\right)
\end{aligned}
$$


As

$$
\rho^{2}=x_{d}^{2}+y_{d}^{2}
$$

$\rho^{2} / \sigma_{d}^{2}$ follows the noncentral chi-square distribution [54] where

$$
P\left(\rho^{2} / \sigma_{d}^{2}\right)=\frac{1}{2} \exp \left(-\frac{\rho^{2} / \sigma_{d}^{2}+\lambda}{2}\right)\left(\frac{\rho^{2}}{\sigma_{d}^{2} \lambda}\right)^{k / 4-1 / 2} I_{k / 2-1}\left(\sqrt{\lambda \rho^{2} / \sigma_{d}^{2}}\right)
$$

where $k$ is the number of degree of freedom and $k=2$ here, $\lambda=\left(\Delta x^{2}+\Delta y^{2}\right) / \sigma_{d}^{2}, I_{v}(z)$ is the modified Bessel function of the first kind. Hence

$$
P\left(\rho^{2}\right)=\frac{1}{2 \sigma_{d}^{2}} \exp \left(-\frac{\rho^{2}+\Delta x^{2}+\Delta y^{2}}{2 \sigma_{d}^{2}}\right) I_{0}\left(\frac{\sqrt{\Delta x^{2}+\Delta y^{2}}}{\sigma_{d}^{2} \rho}\right)
$$

and the overall fading pdf can be calculated by 2.6 and 2.10 .

\subsubsection{Beam Wander Plus Transceiver Induced Extra Pointing Error}

For the untracked or slow tracked FSO communication system, the optical beam random moves due to the turbulence induced beam wander and the possible transceiver induced extra pointing error. The distribution of $\left(x_{d}, y_{d}\right)$ is consisted of two random variable. One is the two dimensional beam wander $\left(x_{b}, y_{b}\right)$ and the other is the extra random pointing error $\left(x_{p}, y_{p}\right)$. And

$$
\begin{aligned}
& x_{d}=x_{b}+x_{p} \\
& y_{d}=y_{b}+y_{p}
\end{aligned}
$$

Assuming beam wander and extra pointing error are independent, denote $P_{x d}$ as the pdf of 
the overall displacement in $x$ direction, $P_{x b}$ and $P_{x p}$ as the pdf of the beam wander and extra pointing error in $x$ direction, respectively,

$$
P_{x d}=P_{x b} * P_{x p}
$$

where $*$ denotes convolution.

Denote $P_{y d}$ as the pdf of the overall pointing error in $y$ direction, $P_{y b}$ and $P_{y p}$ as the pdf of the beam wander and extra pointing error in $y$ direction,

$$
P_{y d}=P_{y b} * P_{y p}
$$

As

$$
\rho^{2}=x_{d}^{2}+y_{d}^{2}
$$

hence

$$
P_{\rho^{2}}=P_{x_{d}^{2}} * P_{y_{d}^{2}}
$$

As

$$
\begin{aligned}
& P\left(x_{d}^{2}\right) d x_{d}^{2}=P\left(\left|x_{d}\right|\right) d\left(\left|x_{d}\right|\right) \\
& P\left(y_{d}^{2}\right) d y_{d}^{2}=P\left(\left|y_{d}\right|\right) d\left(\left|y_{d}\right|\right)
\end{aligned}
$$

where $|\cdot|$ denotes the absolute value. Hence

$$
\begin{aligned}
& P\left(x_{d}^{2}\right)=\frac{P\left(\left|x_{d}\right|\right)}{2\left|x_{d}\right|} \\
& P\left(y_{d}^{2}\right)=\frac{P\left(\left|y_{d}\right|\right)}{2\left|y_{d}\right|}
\end{aligned}
$$

where $P\left(\left|x_{d}\right|\right), P\left(\left|y_{d}\right|\right)$ can be calculated from $P\left(x_{d}\right), P\left(y_{d}\right)$. 


\subsubsection{Beam Wander}

Previous studies show that the atmospheric turbulence induced beam wander $\left(x_{b}, y_{b}\right)$ is a two dimensional Gaussian random variable with zero mean and standard deviation $\sigma_{b}$, where

$$
\begin{aligned}
& x_{b} \sim N\left(0, \sigma_{b}^{2}\right) \\
& y_{b} \sim N\left(0, \sigma_{b}^{2}\right)
\end{aligned}
$$

and

$$
\sigma_{b}^{2}=k C_{n}^{2} D_{0}^{-1 / 3} L^{3}
$$

where $C_{n}^{2}$ is the refractive-index structure parameter, $D_{0}$ is the transmitter aperture diameter, $L$ is the propagation distance, $k$ is the index. The geometrical optics method shows the $k=0.97$ for a collimated uniform beam [23]. $k$ is determined to be 0.76 for collimated Gaussian beam [21].

\subsubsection{Two Dimensional Gaussian Random Model}

The two dimensional Gaussian random model is wildly adopted in the inter-satellite optical communication and urban FSO communication [17], [48]. In this model, the extra pointing error follows independent two dimensional Gaussian distribution in both $x$ and $y$ directions with misalignment $(\Delta x, \Delta y)$ and variance $\sigma_{p}$, where

$$
\begin{aligned}
& x_{p} \sim N\left(\Delta x, \sigma_{p}^{2}\right) \\
& y_{p} \sim N\left(\Delta y, \sigma_{p}^{2}\right)
\end{aligned}
$$


Hence

$$
\begin{aligned}
& x_{d} \sim N\left(\Delta x, \sigma_{d}^{2}\right) \\
& y_{d} \sim N\left(\Delta y, \sigma_{d}^{2}\right)
\end{aligned}
$$

where

$$
\sigma_{d}^{2}=\sigma_{b}^{2}+\sigma_{p}^{2}
$$

Hence $P(\rho)$ can be directly calculated with 2.15. And the overall fading pdf can be calculated by 2.6 and 2.10 .

\subsubsection{Two Dimensional Uniform Random Model}

In the uniform random model, the extra pointing error follows independent uniform distribution in both $x$ and $y$ directions with misalignment $(\Delta x, \Delta y)$ and the maximum sway value $s_{x}$ and $s_{y}$, respectively, where

$$
\begin{aligned}
& x_{p} \sim U\left(\Delta x-s_{x}, \Delta x+s_{x}\right) \\
& y_{p} \sim U\left(\Delta y-s_{y}, \Delta y+s_{y}\right)
\end{aligned}
$$

Hence

$$
P\left(x_{p}\right)= \begin{cases}\frac{1}{2 s_{x}} & , \Delta x-s_{x} \leq x_{p} \leq \Delta x+s_{x} \\ 0 & , \text { elsewhere }\end{cases}
$$




$$
P\left(y_{p}\right)= \begin{cases}\frac{1}{2 s_{y}} & , \Delta y-s_{y} \leq y_{p} \leq \Delta y+s_{y} \\ 0 & , \text { elsewhere }\end{cases}
$$

Then overall fading pdf can be calculated by 2.18, 2.19, 2.22, 2.23, 2.21, 2.6 and 2.10.

\subsubsection{Sine Sway Model}

In the sine sway model, the relative movement of transmitter and receiver is modeled as one dimensional sine sway with misalignment $(\Delta x, \Delta y)$ and the maximum sway value $s$. As the two dimensional Gaussian beam wander is cyclic symmetrical, without losing generality, the sine sway is assumed to be along $x$ direction.

As the cumulative distribution function of the sine sway is the arcsine function, the probability distribution of the sine sway $x_{p}$ follows the arcsine distribution, which is a special case of the beta distribution with $\alpha=\beta=1 / 2$ [55]. With maximum sway value $s$ in $x$ direction,

$$
\begin{aligned}
P\left(x_{p}\right) & =\frac{1}{2 s} \times \frac{1}{\pi \sqrt{\frac{x_{p}-\Delta x+s}{2 s}\left(1-\frac{x_{p}-\Delta x+s}{2 s}\right)}} \\
& =\frac{1}{\pi \sqrt{s^{2}-(x-\Delta x)^{2}}}, \quad \Delta x-s<x_{p}<\Delta x+s \\
P\left(y_{p}\right) & =\delta(\Delta y)
\end{aligned}
$$

Then the overall fading pdf can be calculated by 2.18, 2.19, 2.22, 2.23, 2.21, 2.6 and 2.10. 


\subsection{Simulation Setup}

Direct large-scale numerical wave-optics simulations are conducted to verify the proposed analytical method under different atmospheric turbulence strengths, propagation distances, and pointing error models. In the simulations, the Gaussian laser beam is assumed to propagate along a horizontal direction. The Gaussian laser beam radius is $5 \mathrm{~cm}$ at the transmitter plane. The laser wavelength is $\lambda=1.54 \times 10^{-6} \mathrm{~m}$. The aerosol scattering effects caused by haze, rain, snow, and fog are not considered. And the atmospheric turbulence is simulated by 10 to 12 phase screens uniformly placed between the transmitter and the receiver apertures. The Kolmogorov spectrum is used to generate the phase screens. The grid size of the phase screen ranges from $1024 \times 1024$ to $2048 \times 2048$. The resolution of the phase screen ranges from $0.625 \mathrm{~mm}$ to $2.5 \mathrm{~mm}$. The resolutions are much smaller than the corresponding coherence radius and satisfy the restrictions to accurately simulate the wave propagation [56]. Three atmospheric turbulence channels are studied. They are $1.6 \mathrm{Km}$ (1 mile) channel with $C_{n}^{2}=5 \times 10^{-15} \mathrm{~m}^{-2 / 3} ; 4 \mathrm{Km}(2.5$ miles $)$ channel with $C_{n}^{2}=5 \times 10^{-15} \mathrm{~m}^{-2 / 3}$; And $0.4 \mathrm{Km}(0.25$ mile $)$ channel with $C_{n}^{2}=1 \times 10^{-13} \mathrm{~m}^{-2 / 3}$. Table 2.1 lists the corresponding coherence radius and the Rytov variance of each channel. The Rytov variance ranges from 0.24 to 1.27 . The simulations cover both weak and strong turbulence regimes. The coherence radius ranges from $1.6 \mathrm{~cm}$ to $2.4 \mathrm{~cm}$. The corresponding receiver aperture diameter ranges from point-like $0 \mathrm{~cm}$ to $20 \mathrm{~cm}$. The point-like $0 \mathrm{~cm}$ receiver aperture is the smallest receiver aperture that can be achieved in the simulation. It contains only single pixel. As the simulation resolution $d x$ ranges from $0.625 \mathrm{~mm}$ to $2.5 \mathrm{~mm}$, it is much smaller than the corresponding coherence radius. It can be regarded to a point in the optical field. Hence the receiver aperture size ranges from much smaller than the coherence radius to much larger than the coherence radius. The wave-optics simulations are divided to two sets. The first set of the simulations provide the fast-tracked beam profile and the fast-tracked on-axis and off-axis fading pdfs information for each of the three optical channel. The simulation parameters are listed in table 2.2. Then the overall fading pdfs with pointing error 
are calculated using the analytical method based on the obtained channel information and pointing error models. The second set of simulations directly simulate the overall fading pdfs with corresponding pointing error for each channel to verify the analytical method. Both fast-tracking residual model and beam wander plus transceiver induced extra pointing error model are studied. Three common extra pointing error models, the Gaussian random model, the uniform random model, and the sine sway model are performed in these turbulence channels. Table 2.3 lists the parameters in the direct wave-optics simulations of the overall fading pdfs. The overall fading pdfs are studied for both with and without misalignment cases. For Gaussian random model, the standard deviation $\sigma_{d}$ of the overall pointing error ranges from $1.29 \mathrm{~cm}$ to $2.57 \mathrm{~cm}$. For uniform random model the maximum sway amplitude in $x$ and $y$ directions are $s_{x}=2 \mathrm{~cm}, s_{y}=2 \mathrm{~cm}$. For sine sway model, $s_{x}=2 \mathrm{~cm}$, $s_{y}=0 \mathrm{~cm}$.

Table 2.1: Rytov variance and coherence radius.

\begin{tabular}{cccc}
\hline $\begin{array}{c}L \\
(\mathrm{Km})\end{array}$ & $\begin{array}{c}C_{n}^{2} \\
\left(\mathrm{~m}^{-2 / 3}\right)\end{array}$ & $\sigma_{R}^{2}$ & $\begin{array}{c}r_{0} \\
(\mathrm{~cm})\end{array}$ \\
\hline 0.4 & $1 \times 10^{-13}$ & 0.37 & 1.6 \\
\hline 1.6 & $5 \times 10^{-15}$ & 0.24 & 4.2 \\
\hline 4 & $5 \times 10^{-15}$ & 1.27 & 2.4 \\
\hline
\end{tabular}

Table 2.2: Simulation parameters for channel information.

\begin{tabular}{cccccc}
\hline $\begin{array}{c}L \\
(\mathrm{Km})\end{array}$ & $\begin{array}{c}C_{n}^{2} \\
\left(\mathrm{~m}^{-2 / 3}\right)\end{array}$ & $\mathrm{N}$ & $\begin{array}{c}d x \\
(\mathrm{~mm})\end{array}$ & $\begin{array}{c}M \\
\left(10^{6}\right)\end{array}$ & $N_{p h z}$ \\
\hline 0.4 & $1 \times 10^{-13}$ & 2048 & 0.625 & 0.12 & 10 \\
\hline 1.6 & $5 \times 10^{-15}$ & 1024 & 2.5 & 0.31 & 10 \\
\hline 4 & $5 \times 10^{-15}$ & 1024 & 2.5 & 0.67 & 12 \\
\hline
\end{tabular}


Table 2.3: Simulation parameters for overall fading pdf.

\begin{tabular}{cccccccccccccc}
\hline $\begin{array}{c}L \\
(\mathrm{Km})\end{array}$ & $\begin{array}{c}\text { Pointing error } \\
\text { model }\end{array}$ & $\begin{array}{c}C_{n}^{2} \\
\left(\mathrm{~m}^{-2 / 3}\right)\end{array}$ & $\begin{array}{c}\Delta x \\
(\mathrm{~cm})\end{array}$ & $\begin{array}{c}\Delta y \\
(\mathrm{~cm})\end{array}$ & $\begin{array}{c}\sigma_{b} \\
(\mathrm{~cm})\end{array}$ & $\begin{array}{c}\sigma_{p} \\
(\mathrm{~cm})\end{array}$ & $\begin{array}{c}\sigma_{d} \\
(\mathrm{~cm})\end{array}$ & $\begin{array}{c}s_{x} \\
(\mathrm{~cm})\end{array}$ & $\begin{array}{c}s_{y} \\
(\mathrm{~cm})\end{array}$ & $\begin{array}{c}d x \\
(\mathrm{~mm})\end{array}$ & $\begin{array}{c}M \\
\left(10^{6}\right)\end{array}$ & $N_{p h z}$ \\
\hline 0.4 & Gaussian & $1 \times 10^{-13}$ & 0 & 0 & 0.32 & 1.25 & 1.29 & - & - & 2048 & 0.625 & 0.41 & 10 \\
\hline 1.6 & Gaussian & $5 \times 10^{-15}$ & 0 & 0 & 0.58 & 2.50 & 2.57 & - & - & 1024 & 2.5 & 0.56 & 10 \\
\hline 1.6 & Gaussian & $5 \times 10^{-15}$ & 2.5 & 2.5 & 0.58 & 2.50 & 2.57 & - & - & 1024 & 2.5 & 0.70 & 10 \\
\hline 4 & residual & $5 \times 10^{-15}$ & 0 & 0 & - & - & 2.00 & - & - & 1536 & 1.6 & 0.49 & 12 \\
\hline 4 & Gaussian & $5 \times 10^{-15}$ & 0 & 0 & 2.29 & 1.00 & 2.50 & - & - & 1024 & 2.5 & 1 & 12 \\
\hline 4 & uniform & $5 \times 10^{-15}$ & 0 & 0 & 2.29 & - & 2.29 & 2.00 & 2.00 & 1024 & 2.5 & 1 & 12 \\
\hline 4 & sine & $5 \times 10^{-15}$ & 0 & 0 & 2.29 & 0 & 2.29 & 2.00 & - & 1024 & 2.5 & 1 & 12 \\
\hline
\end{tabular}

\subsection{Simulation Results}

\subsubsection{Channel Information}

For a FSO channel, as shown in 2.10, the fast-tracked beam profile and the fast-tracked onaxis and off-axis fading pdfs contain the information needed to calculate the overall fading pdf with pointing error. In this chapter, the fast-tracked beam profile and the fast-tracked on-axis and off-axis fading pdfs are obtained from numerical simulations. The off-axis fading pdfs are sampled until at least $10 \mathrm{~cm}$ away from the beam weight center with more than 10 sample locations. The sample range covers most of locations the beam may arrive at the receiver plane. For the point-like $0 \mathrm{~cm}$ receiver aperture, the on-axis and off-axis fast-tracked fading pdfs fit well with gamma distribution [57]. The gamma model is used as the fast-tracked on-axis and off-axis fading pdf model for point-like $0 \mathrm{~cm}$ apertures to calculate the overall fading pdfs in this chapter. Hence only fast-tracked on-axis and offaxis variance information is needed to be obtained from simulations. Table 2.4 shows the on-axis fading pdf variance and parts of the off-axis fading pdf variance of the three optical channels. As receiver aperture grows larger, the fast-tracked on-axis and off-axis fading pdfs are directly obtained from the numerical simulations. 
Table 2.4: Fast-tracked fading pdf variance for point-like $0 \mathrm{~cm}$ aperture

\begin{tabular}{cccccc}
\hline \multirow{2}{*}{$\begin{array}{c}C_{n}^{2} \\
\mathrm{Km})\end{array}$} & \multicolumn{4}{c}{ Sample location } \\
\cline { 3 - 6 }$\left(\mathrm{m}^{-2 / 3}\right)$ & on-axis & $3.75 \mathrm{~cm}$ & $7.5 \mathrm{~cm}$ & $10 \mathrm{~cm}$ \\
\hline 0.4 & $1 \times 10^{-13}$ & 0.291 & 0.338 & 0.664 & 1.013 \\
\hline 1.6 & $5 \times 10^{-15}$ & 0.133 & 0.171 & 0.632 & 1.159 \\
\hline 4 & $5 \times 10^{-15}$ & 0.400 & 0.411 & 1.094 & 1.700 \\
\hline
\end{tabular}

\subsubsection{Overall Fading Pdf}

Given the fast-tracked beam profile and the fast-tracked on-axis and off-axis fading pdf information of each channel, the overall fading pdfs are directly calculated with the given pointing error model using the analytical method. The corresponding overall fading pdfs are also obtained from direct wave-optics simulations to verify the analytically calculated results.

\subsubsection{1 $L=1.6 \mathrm{Km}, C_{n}^{2}=5 \times 10^{-15} \mathbf{m}^{-2 / 3}$ Channel}

The Rytov variance of the $1.6 \mathrm{Km}, C_{n}^{2}=5 \times 10^{-15} \mathrm{~m}^{-2 / 3}$ channel is 0.24 . The radius of the fast-tracked beam profile is $5.26 \mathrm{~cm}$ (place with $1 / e^{2}$ of maximum beam intensity). In this weak turbulence channel, the analytical results are calculated for the beam wander plus Gaussian extra pointing error model under both with and without misalignment cases based on the fast-tracked beam profile and the fast-tracked on-axis and off-axis fading pdfs. Figure 2.2 shows the calculated analytical fading pdfs for beam wander plus Gaussian pointing error model with zero misalignment and $\sigma_{p}=2.5 \mathrm{~cm}$ in both $x$ and $y$ directions. Figure 2.3 shows the calculated analytical fading pdfs for beam wander plus Gaussian pointing error 
model with $2.5 \mathrm{~cm}$ misalignment and $\sigma_{p}=2.5 \mathrm{~cm}$ in both $x$ and $y$ directions. The corresponding GG and LN curves are also shown for comparison. The parameters $\alpha$ and $\beta$ for the GG model are obtained by doing best fit to the simulated overall fading pdf data while keeping the same fading variance [38]. The parameter for LN model is obtained from the simulated fading variance. The figures show the fading pdfs of $10 \log _{10}(h)$, where $h$ is the normalized received beam power, as the deep fading tail is more critical to determine the system BER performance. As shown in table 2.1, the coherence radius of this turbulence channel is $4.2 \mathrm{~cm}$. The point-like $0 \mathrm{~cm}$ receiver aperture is much smaller than the coherence radius. The receiver aperture with $10 \mathrm{~cm}$ diameter is closed to the size of the fast-tracked beam spot. The $20 \mathrm{~cm}$ receiver aperture is much larger than the coherence radius. It is shown that the analytical results accurately fit the direct wave-optics simulation results under all the studied receiver aperture sizes in both pointing error cases. And the best fitted GG and LN fading pdfs deviate from the simulated fading pdf at both left and right tails from point-like $0 \mathrm{~cm}$ receiver aperture to the $20 \mathrm{~cm}$ receiver aperture. For Gaussian extra pointing error model with $0 \mathrm{~cm}$ misalignment and $\sigma_{p}=2.5 \mathrm{~cm}$ case, the overall beam random displacement standard deviation is $\sigma_{d}=2.57 \mathrm{~cm}$. With point-like $0 \mathrm{~cm}$ receiver aperture, both simulated fading probability density and analytical fading probability density remain a little higher than $10^{-5}$ at $-40 \mathrm{~dB}$. The best fitted GG pdf model estimates the probability density reduces to $10^{-5}$ at about $-25 \mathrm{~dB}$ power fading. The $\mathrm{LN}$ pdf model reaches $10^{-5}$ at only about $-13 \mathrm{~dB}$ power fading. With $10 \mathrm{~cm}$ receiver aperture, both GG and LN pdf models reach probability density $10^{-5}$ at about $-7 \mathrm{~dB}$ while the simulated fading pdf and the analytical fading pdf keep higher than $10^{-5}$ until more than $-20 \mathrm{~dB}$ power fading. With $20 \mathrm{~cm}$ receiver aperture, large part of the instant random laser beam spot is covered by the receiver aperture, hence the fading is relatively weak. Both the simulated fading pdf and the analytical fading pdf reach $10^{-5}$ at about $-8 \mathrm{~dB}$ power fading. The $\mathrm{LN}$ model reaches $10^{-5}$ at about only $-1.5 \mathrm{~dB}$. The GG model approaches to the $\mathrm{LN}$ fading pdf model in this case. It also should be noticed that both GG and LN pdf models estimate higher probability density to receive large power (the right tails) than the simulated fading 
pdf and the analytical fading pdf in all the receiver aperture cases.

When the misalignment increases to $2.5 \mathrm{~cm}$ in both $x$ and $y$ directions, the chance of random beam deviating from the receiver aperture increases. With the same overall random displacement standard deviation $\sigma_{d}$, the probability density of the FSO channel getting deep fading becomes higher. With point-like $0 \mathrm{~cm}$ receiver aperture, the probability density of the FSO communication system suffering from $-40 \mathrm{~dB}$ power fading increases from around $10^{-5}$ to about $2 \times 10^{-4}$. And the probability density of the system to get $-51 \mathrm{~dB}$ deep power fading is $10^{-5}$. With $10 \mathrm{~cm}$ receiver aperture, the probability density of the system getting $-20 \mathrm{~dB}$ power fading increases from about $3 \times 10^{-5}$ to about $5 \times 10^{-4}$. For $20 \mathrm{~cm}$ receiver aperture, the chance of part of the random beam moving out the receiver aperture increases compared to no misalignment case. The probability density of the system suffering $-7 \mathrm{~dB}$ power fading increases from about $2 \times 10^{-5}$ to about $5 \times 10^{-4}$. And the probability density of suffering $-14 \mathrm{~dB}$ power fading increases to higher than $1 \times 10^{-5}$. In this pointing error case, the GG pdf model and the LN pdf model still overestimate the probability density of receiving high beam power and hence optimistically estimate the probability density of the system experiencing deep fading.

\subsubsection{2 $L=4 \mathrm{Km}, C_{n}^{2}=5 \times 10^{-15} \mathbf{m}^{-2 / 3}$ Channel}

The radius of the fast-tracked beam profile grows to $6.34 \mathrm{~cm}$ at the receiver plane for the $4 \mathrm{Km}$ channel. The Rytov variance is 1.27 . It is in strong atmospheric turbulence regime. The coherence radius is $2.4 \mathrm{~cm}$. The receiver aperture diameter ranges from $0 \mathrm{~cm}$ to $20 \mathrm{~cm}$. Based on the fast-tracked beam profile and the fast-tracked on-axis and off-axis fading pdfs information, the overall fading pdfs are calculated for four different random overall pointing error models. They are the fast-tracking residual pointing error model, the beam wader plus Gaussian random pointing error model, the beam wader plus uniform random pointing error model, and the beam wander plus sine sway model. 


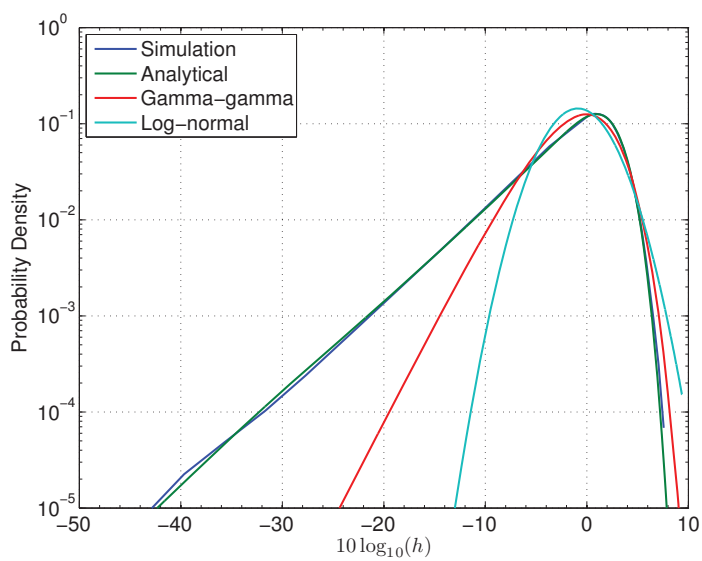

(a) $D=0 \mathrm{~cm}$

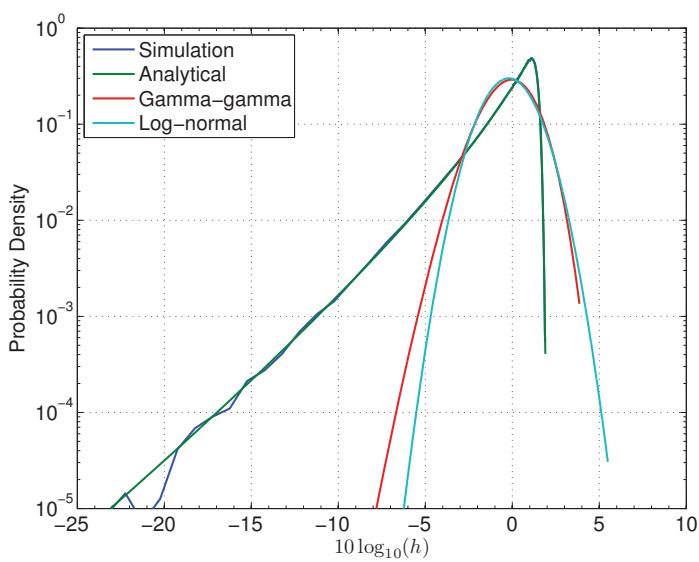

(c) $D=10 \mathrm{~cm}$

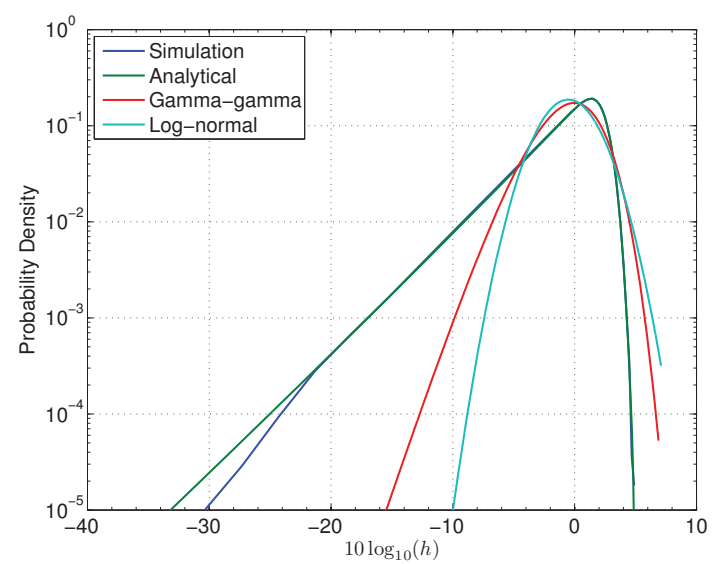

(b) $D=5 \mathrm{~cm}$

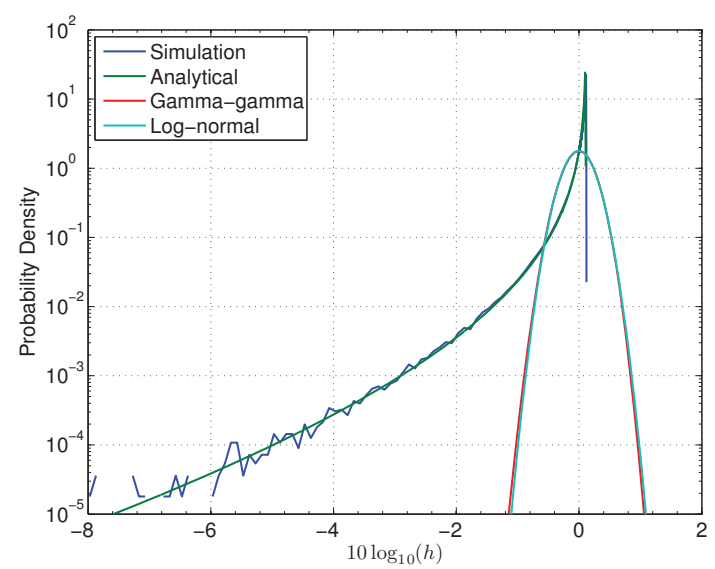

(d) $D=20 \mathrm{~cm}$

Figure 2.2: Comparison for the analytical curve with the direct wave-optics simulation curve. $C_{n}^{2}=5 \times 10^{-15} \mathrm{~m}^{-2 / 3}$. $L=1.6 \mathrm{Km}$. Gaussian extra pointing error model. $\sigma_{b}=0.58 \mathrm{~cm} . \sigma_{p}=2.5 \mathrm{~cm} . \sigma_{d}=2.57 \mathrm{~cm} . \Delta x=\Delta y=0 \mathrm{~cm} . d_{x}=2.5 \mathrm{~mm}$. Coherence radius is $4.2 \mathrm{~cm}$. Rytov variance is $0.24 .1024 \times 1024$. $0.56 \mathrm{M}$ iterations.

Figure 2.4 shows the comparison of the large-scale direct wave-optics simulated overall fading pdfs and the calculated analytical overall fading pdfs for the $L=4 \mathrm{Km}, C_{n}^{2}=$ $5 \times 10^{-15} \mathrm{~m}^{-2 / 3}$ channel with Gaussian fast-tracking residual pointing error model. The transceiver induced extra pointing error is assumed to be well eliminated. And the beam wander is assumed to be reduced by the fast-tracking system. The fast-tracking residual is assumed to follow two dimensional Gaussian distribution with mean $\Delta x=\Delta y=0 \mathrm{~cm}$ and $\sigma_{p}=2 \mathrm{~cm}$ in both $x$ and $y$ directions. The analytical fading pdfs accurately follow the simulated fading pdfs in all the receiver aperture sizes. For this relatively small random 


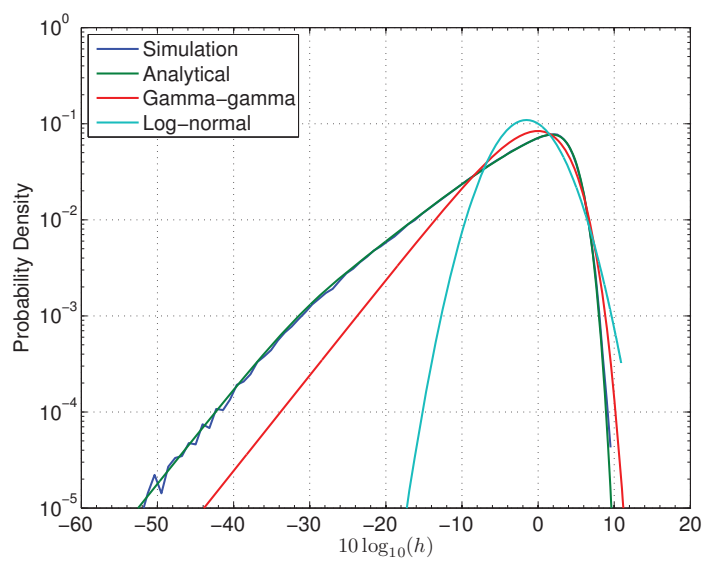

(a) $D=0 \mathrm{~cm}$

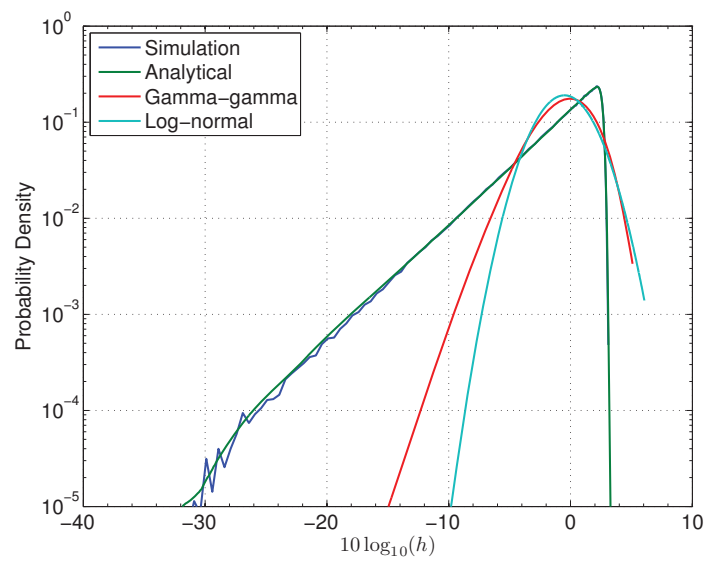

(c) $D=10 \mathrm{~cm}$

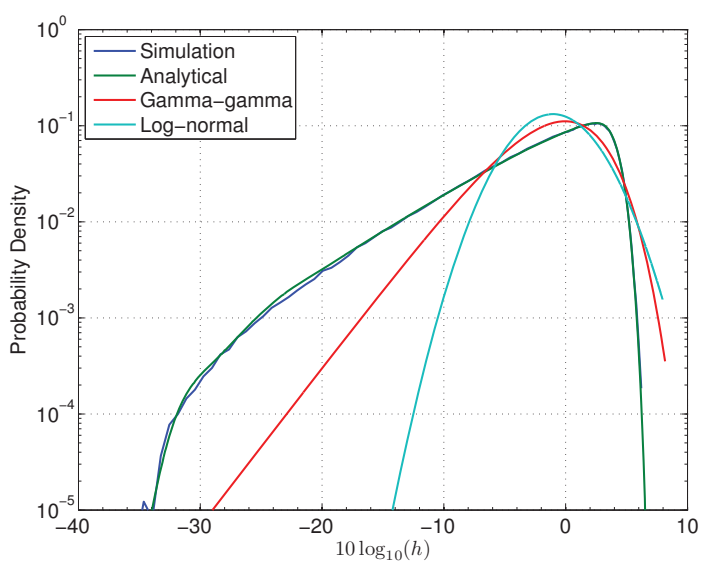

(b) $D=5 \mathrm{~cm}$

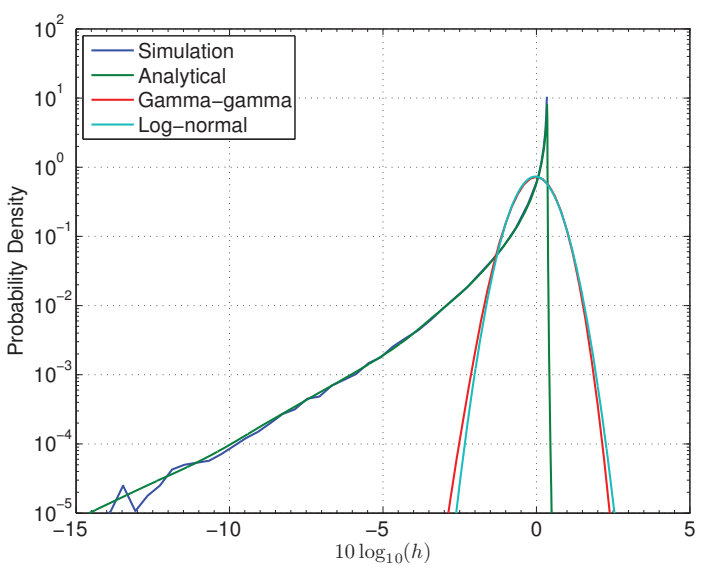

(d) $D=20 \mathrm{~cm}$

Figure 2.3: Comparison for the analytical curve with the direct wave-optics simulation curve. $C_{n}^{2}=5 \times 10^{-15} \mathrm{~m}^{-2 / 3} . L=1.6 \mathrm{Km}$. Gaussian extra pointing error model. $\sigma_{b}=0.58 \mathrm{~cm} . \sigma_{p}=2.5 \mathrm{~cm} . \sigma_{d}=2.57 \mathrm{~cm} . \Delta x=\Delta y=2.5 \mathrm{~cm} . d_{x}=2.5 \mathrm{~mm}$. Coherence radius is $4.2 \mathrm{~cm}$. Rytov variance is $0.24 .1024 \times 1024$. $0.70 \mathrm{M}$ iterations.

pointing error, the GG and LN fading pdf models still fail to accurately estimate the overall fading pdfs.

Figure 2.5 shows overall fading pdfs for beam wander plus extra Gaussian random pointing error model where $\Delta x=\Delta y=0 \mathrm{~cm}$ and $\sigma_{p}=1 \mathrm{~cm}$ in both $x$ and $y$ directions. The beam wander standard deviation $\sigma_{b}=2.29 \mathrm{~cm}$. The total random displacement standard deviation is $\sigma_{d}=2.5 \mathrm{~cm}$. It is shown that with point-like $0 \mathrm{~cm}$ receiver aperture, the probability density of the system getting -50 power fading is around $10^{-5}$. The fading pdf is much less severe 
comparing with the fading pdf (figure 2.3(a)) obtained from the $L=1.6 \mathrm{Km}, \Delta=2.5 \mathrm{~cm}$, $\sigma_{d}=2.57 \mathrm{~cm}$ channel with $0 \mathrm{~cm}$ receiver aperture. This is because of the narrower fasttracked beam profile and the relatively larger misalignment for the $1.6 \mathrm{Km}$ case although the overall random displacement standard deviation is almost the same. The overall fading pdfs with extra uniform random pointing error model and extra sine sway pointing error model in this $4 \mathrm{Km}$ channel are also calculated. Figure 2.6(a) and figure 2.6(b) show the calculated analytical fading pdfs for uniform random model and sine sway model with $0 \mathrm{~cm}$ receiver aperture, respectively. In the uniform random model, the overall pointing error is the turbulence induced two dimensional Gaussian random beam wander combining with the independent two dimensional uniform random extra pointing error with max sway amplitude $s=2 \mathrm{~cm}$ in both $x$ and $y$ directions. In the sine sway model, the overall pointing error is the two dimensional Gaussian beam wander combining with the one dimensional sine sway with max sway amplitude $s=2 \mathrm{~cm}$ in $x$ direction. It is shown in the studied cases, the probability density of system with two dimensional uniform random extra pointing error model suffering from deep fading is a little higher than the corresponding system with the one dimensional sine sway extra pointing error model. In all the pointing error cases, the analytical fading pdfs fit well with the simulated fading pdfs. The GG and LN pdf models deviate from the simulated fading pdf.

\subsubsection{3 $L=0.4 \mathrm{Km}, C_{n}^{2}=1 \times 10^{-13} \mathbf{m}^{-2 / 3}$ Channel}

The overall fading pdfs are also studied for higher $C_{n}^{2}$ case. Figure 2.7 shows the large-scale direct wave-optics simulated fading pdfs and the analytical calculated overall fading pdfs in turbulence channel with $L=0.4 \mathrm{Km}, C_{n}^{2}=1 \times 10^{-13} \mathrm{~m}^{-2 / 3}$. The receiver aperture size ranges from point-like $0 \mathrm{~cm}$ aperture to $20 \mathrm{~cm}$ receiver aperture. The coherence radius is $1.6 \mathrm{~cm}$. The pointing error model is the beam wander plus extra Gaussian random pointing error model with zero misalignment and $\sigma_{p}=1.25 \mathrm{~cm}$. The beam wander standard deviation $\sigma_{b}=0.32 \mathrm{~cm}$. The total random displacement standard deviation $\sigma_{d}=1.29 \mathrm{~cm}$. The 


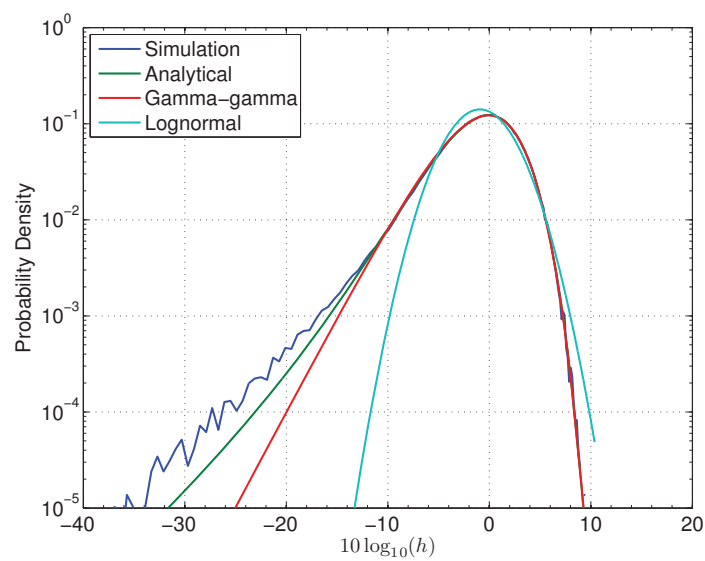

(a) $D=0 \mathrm{~cm}$

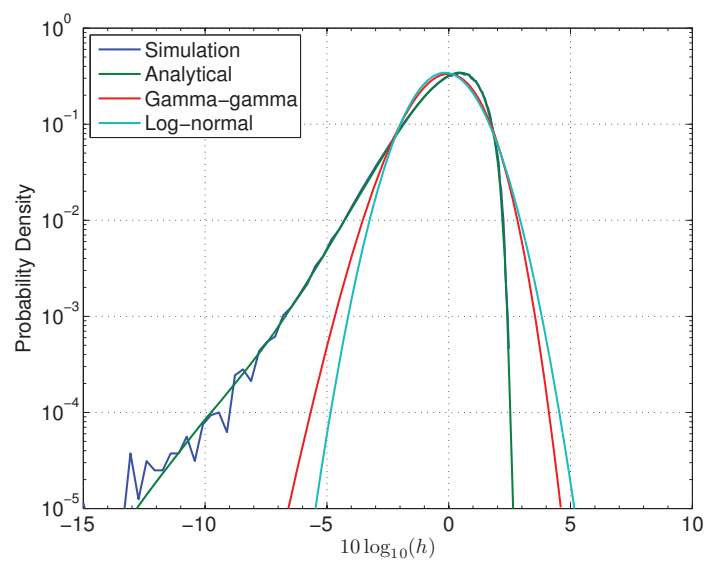

(c) $D=10 \mathrm{~cm}$

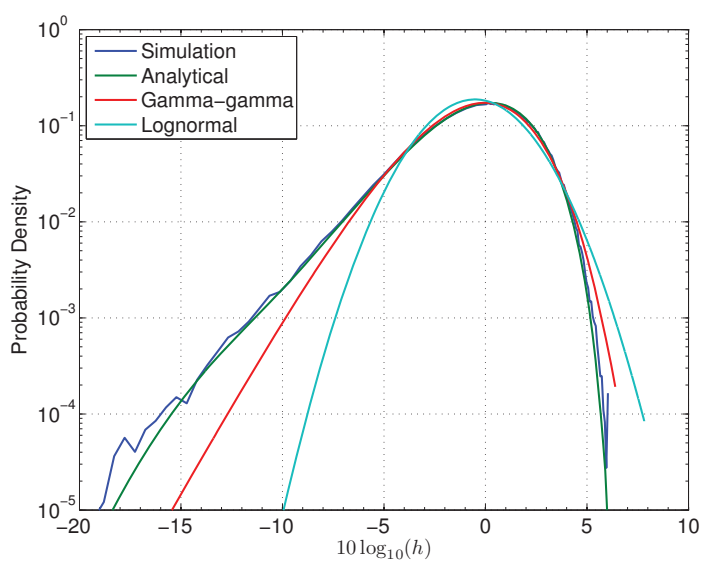

(b) $D=5 \mathrm{~cm}$

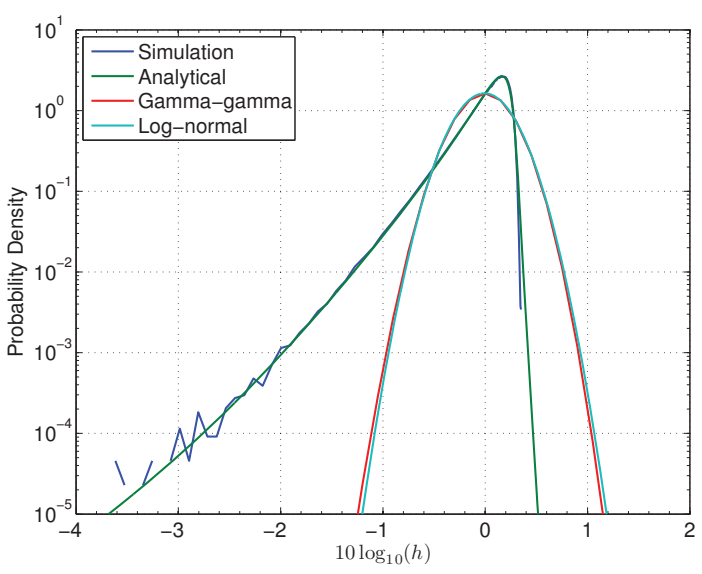

(d) $D=20 \mathrm{~cm}$

Figure 2.4: Comparison for the analytical curve with the direct wave-optics simulation curve. $C_{n}^{2}=5 \times 10^{-15} \mathrm{~m}^{-2 / 3} . L=4 \mathrm{Km}$. Gaussian tracking residual error model. $\sigma_{d}=2 \mathrm{~cm} . \Delta x=\Delta y=0 \mathrm{~cm} . d_{x}=1.6 \mathrm{~mm}$. Coherence radius is $2.4 \mathrm{~cm}$. Rytov variance is $1.27 .1024 \times 1024$. $0.49 \mathrm{M}$ iterations.

analytical fading pdfs also fit well with the simulated fading pdf in all the aperture sizes in this high $C_{n}^{2}$ case. 


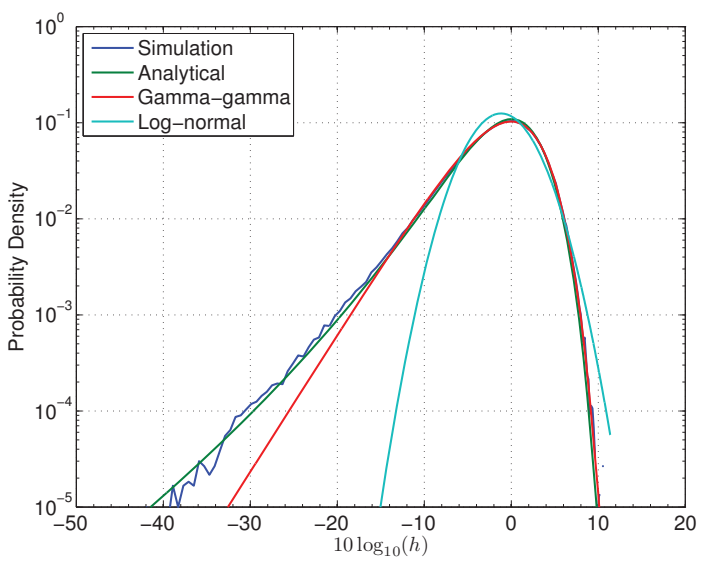

(a) $D=0 \mathrm{~cm}$

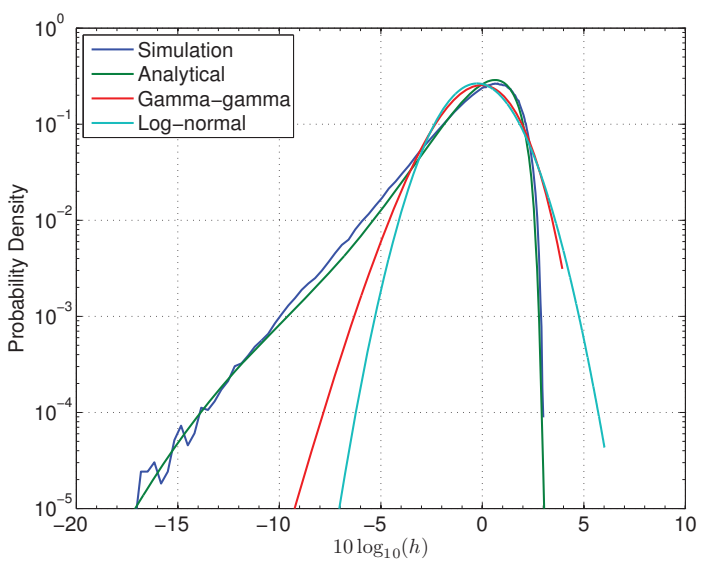

(c) $D=10 \mathrm{~cm}$

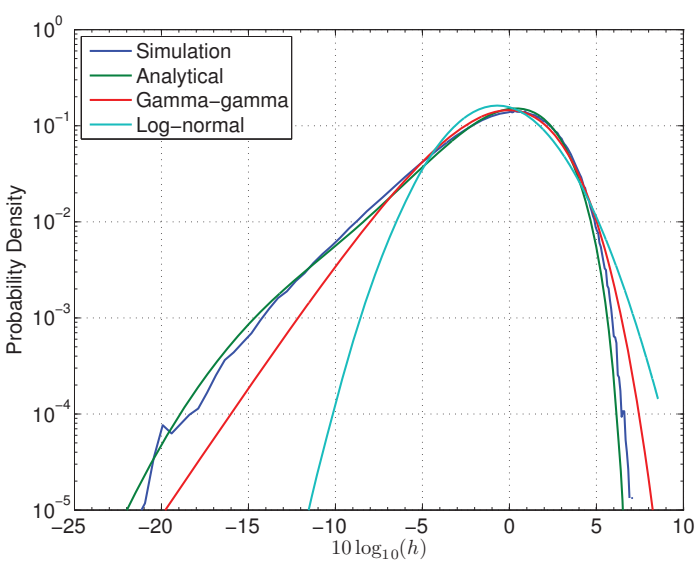

(b) $D=5 \mathrm{~cm}$

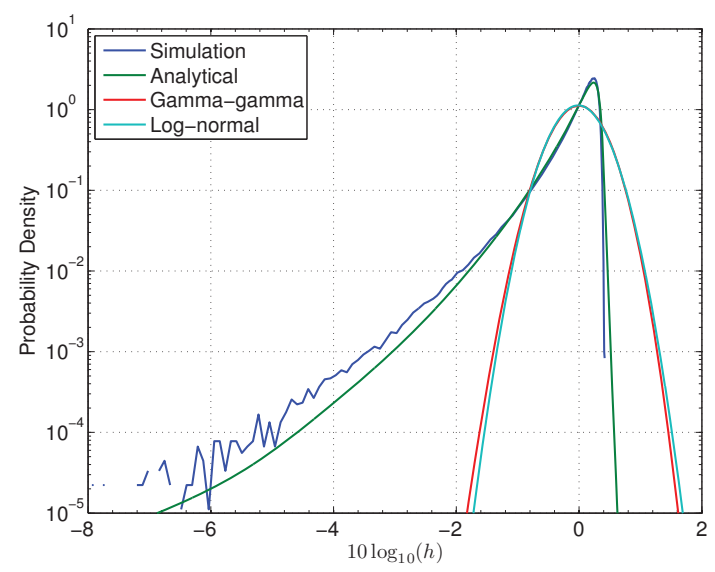

(d) $D=20 \mathrm{~cm}$

Figure 2.5: Comparison for the analytical curve with the direct wave-optics simulation curve. $C_{n}^{2}=5 \times 10^{-15} \mathrm{~m}^{-2 / 3} . L=4 \mathrm{Km}$. Gaussian extra pointing error model. $\sigma_{b}=2.29 \mathrm{~cm} . \sigma_{p}=1 \mathrm{~cm} . \sigma_{d}=2.5 \mathrm{~cm} . \Delta x=\Delta y=0 \mathrm{~cm} . d_{x}=2.5 \mathrm{~mm}$. Coherence radius is $2.4 \mathrm{~cm}$. Rytov variance is 1.27 . $1024 \times 1024$. $1 \mathrm{M}$ iterations.

\subsubsection{Average BER and Outage Probability}

To evaluate the influence of the fading pdf to the FSO communication system performance, the BER and the outage probability are studied. Assume the system model is

$$
y=h x+n
$$




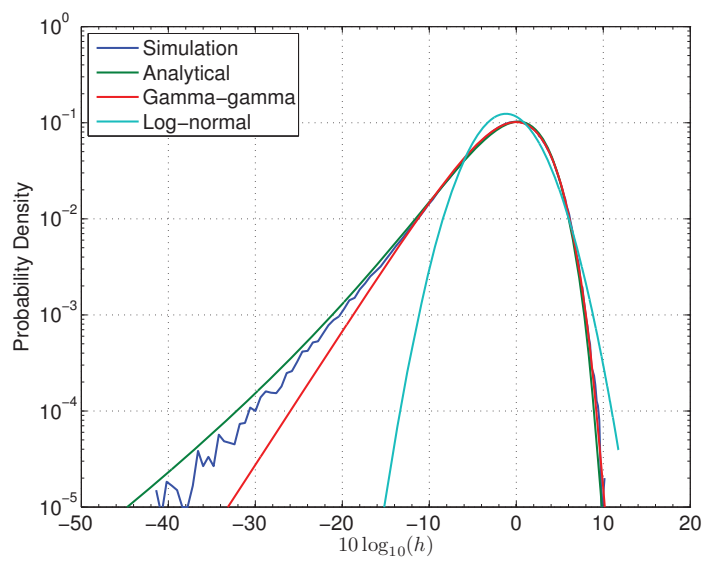

(a) Uniform random model $D=0 \mathrm{~cm}$

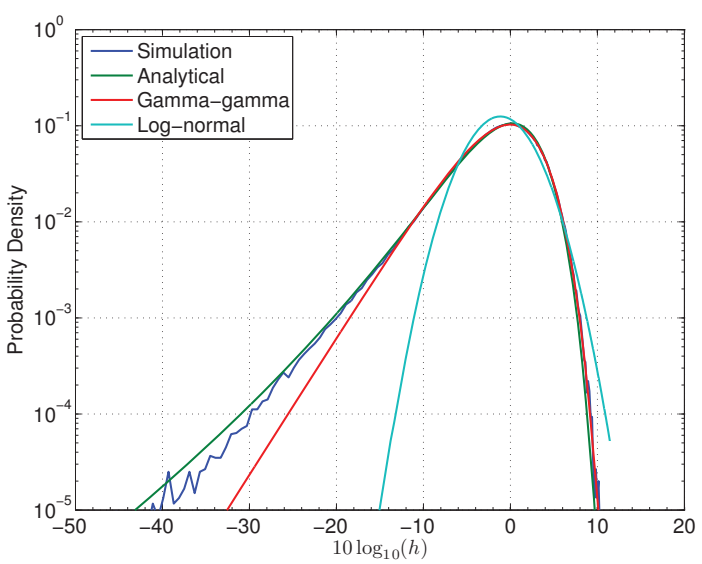

(b) Sine sway model $D=0 \mathrm{~cm}$

Figure 2.6: Comparison for the analytical curve with the direct wave-optics simulation curve. $C_{n}^{2}=5 \times 10^{-15} \mathrm{~m}^{-2 / 3} . L=4 \mathrm{Km}$. Uniform random model and sine sway model. $\sigma_{b}=2.29 \mathrm{~cm} . s=2 \mathrm{~cm} . d_{x}=2.5 \mathrm{~mm}$. Coherence radius is $2.4 \mathrm{~cm}$. Rytov variance is $1.27 .1024 \times 1024.1 \mathrm{M}$ iterations.

where $y$ is the received instantaneous signal, $h$ is the normalized channel fading, $x$ is the average received signal, $n$ is the equivalent Gaussian noise, and $n \sim N\left(0, \sigma_{n}^{2}\right)$. Assume the signal is modulated by OOK modulation where

$$
x=\left\{\begin{array}{cc}
\bar{x}, & \text { send } 1 \\
0, & \text { send } 0
\end{array}\right.
$$

where $\bar{x}$ is the average received power when ' 1 ' is transmitted. Assume the probabilities to send signal ' 0 ' and signal ' 1 ' are equal, hence $P(x=0)=P(x=\bar{x})=1 / 2$. Assume the receiver has the knowledge of the instant channel state $h$, given Gaussian noise $n$, the optimal threshold $T_{h}$ that minimize the error probability is $T_{h}=h \bar{x} / 2$. Denote

$$
A=\frac{1}{2} \bar{x},
$$

the instantaneous error probability $P_{e}(h)$ after optimal thresholding is [54] 


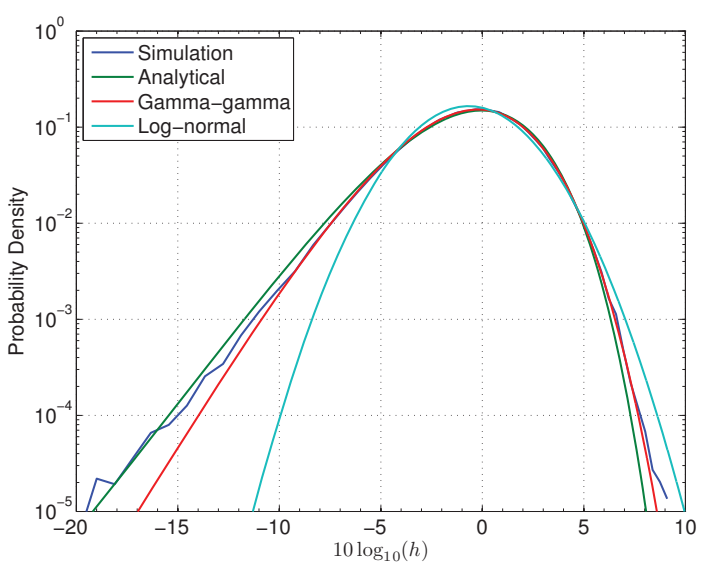

(a) $D=0 \mathrm{~cm}$

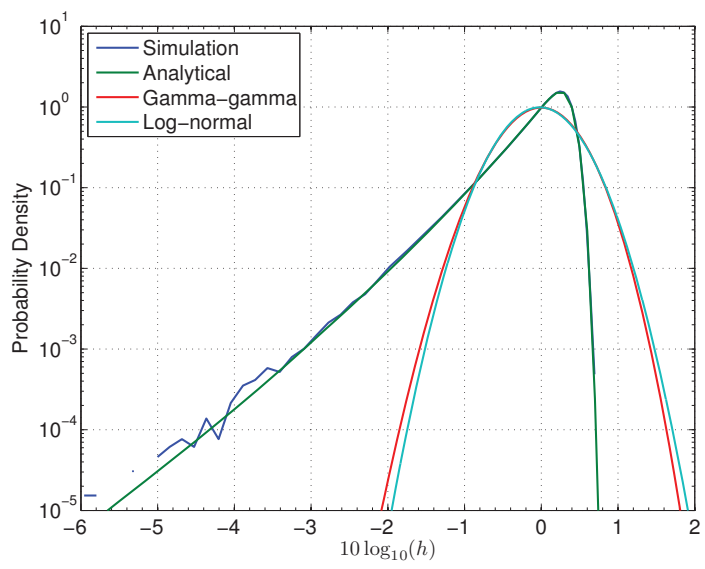

(c) $D=10 \mathrm{~cm}$

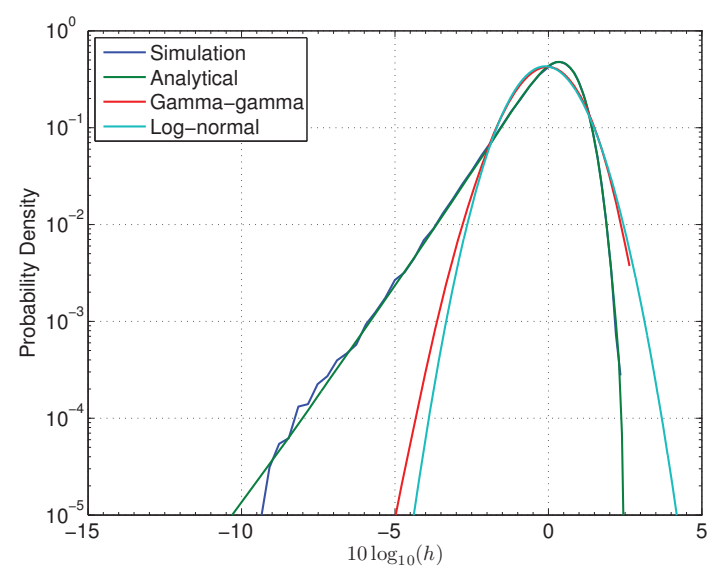

(b) $D=5 \mathrm{~cm}$

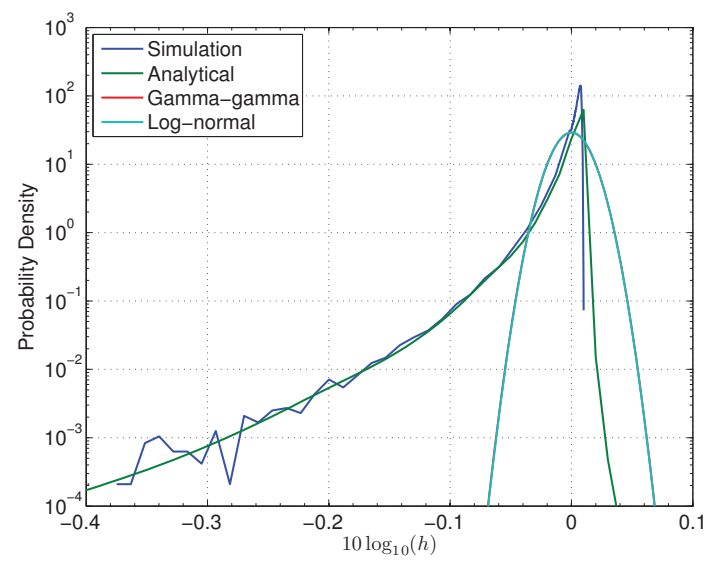

(d) $D=20 \mathrm{~cm}$

Figure 2.7: Comparison for the analytical curve with the direct wave-optics simulation curve. $C_{n}^{2}=1 \times 10^{-13} \mathrm{~m}^{-2 / 3} . L=0.4 \mathrm{Km}$. Gaussian extra pointing error. $\sigma_{b}=0.32 \mathrm{~cm} . \quad \sigma_{p}=1.25 \mathrm{~cm} . \quad \sigma_{d}=1.29 \mathrm{~cm} . \quad \Delta x=\Delta y=0 \mathrm{~cm} . \quad d_{x}=0.625 \mathrm{~mm}$. Coherence radius is $1.6 \mathrm{~cm}$. Rytov variance is 0.37 . $2048 \times 2048$. $0.41 \mathrm{M}$ iterations.

$$
P_{e}(h)=Q\left(\frac{h \bar{x}}{2 \sigma_{n}}\right)=Q\left(\frac{h A}{\sigma_{n}}\right)
$$

Hence

$$
\bar{P}_{e}=\int P_{e}(h) P(h) d h
$$


where $\bar{P}_{e}$ is the average BER.

Assuming $\sigma_{n}^{2}=1$, the average BERs calculated from simulated fading pdf, analytical fading pdf, best fitted GG pdf and LN pdf are compared. Figure 2.8 shows the estimated BER performance for the FSO channel with $L=1.6 \mathrm{Km}$ and $C_{n}^{2}=5 \times 10^{-15} \mathrm{~m}^{-2 / 3}$. The pointing error model is beam wander plus transceiver induced extra Gaussian random pointing error model with $\Delta=2.5 \mathrm{~cm}$ and $\sigma_{p}=2.5 \mathrm{~cm}$ in both $x$ and $y$ directions. The corresponding fading pdfs are shown in figure 2.3. It is shown that the analytical method accurately estimates the system BER performance. Both the best fitted GG model and LN model underestimate the system BER for all the studied receiver aperture sizes. LN model predicts the most underestimated BER performance in all the fading pdf models. The BER estimated from the best fitted GG model lies between the BER obtained from LN model and the BER obtained from simulated fading pdf. When the aperture size grows, the BER estimated from GG model approaches the BER estimated from the LN model. And both the BERs still deviate from that estimated by the simulated fading pdf and analytical fading pdf. For the point-like $0 \mathrm{~cm}$ receiver aperture, with the average received energy $10 \log _{10}(A)=18 \mathrm{~dB}$, the BER estimated by simulated fading pdf and the BER estimated by the analytical fading pdf are both about $1.8 \times 10^{-2}$. The BER estimated by the best fitted GG model is about $8.6 \times 10^{-4}$. The BER estimated by the corresponding LN model is only about $1 \times 10^{-5}$. Hence the BER estimated from LN model is about 1800 times lower than the actual BER obtained from simulation. Although best fitted GG model has a much better estimation than the LN model, it still about only $4.8 \%$ of that estimated by the simulation. And even the BER estimated from the GG model deviates from the simulated BER curve since BER $=2 \times 10^{-1}$. For $10 \mathrm{~cm}$ receiver aperture, when $10 \log _{10}(A)=11.6 \mathrm{~dB}$, the BER estimated by the simulated fading pdf and that by the analytical fading pdf are both about $8.3 \times 10^{-3}$. The BER estimated by the GG model is $11.2 \times 10^{-5}$. The BER estimated by the LN model is only $1 \times 10^{-5}$. When the aperture size grows to $20 \mathrm{~cm}$, the fading is less severe $(2.3(\mathrm{~d}))$. In this case, with $10 \log _{10}(A)=6.9 \mathrm{~dB}$, the BER estimated by LN model is still about only 
$1.4 \%$ of that obtained by either simulated fading pdf or the analytical fading pdf. The BER estimated by the GG model is almost identical to the BER estimated by the LN fading pdf model. When receiver aperture size grows even larger, the fading pdf obtained from each model becomes quite narrow. The BER approaches the no fading Gaussian channel BER performance (not shown here). And they are not sensitive to a particular fading pdf model.

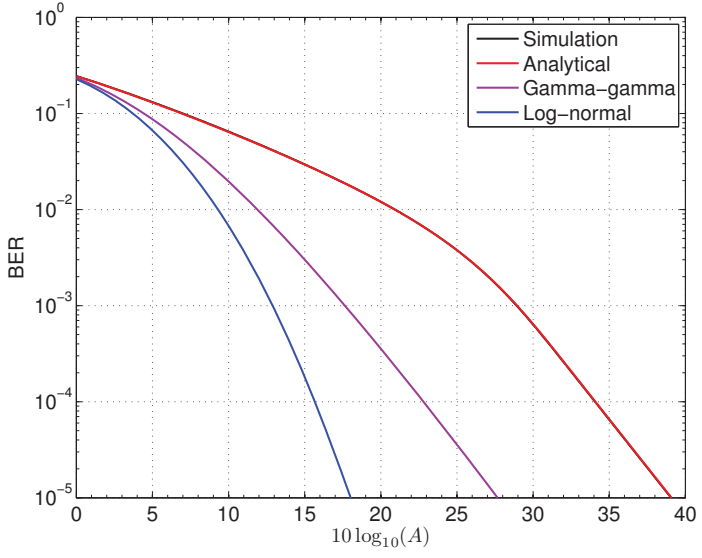

(a) $D=0 \mathrm{~cm}$

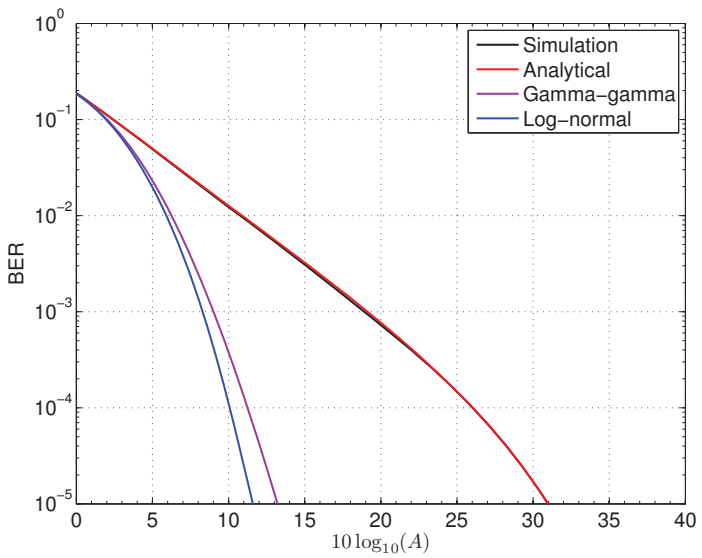

(c) $D=10 \mathrm{~cm}$

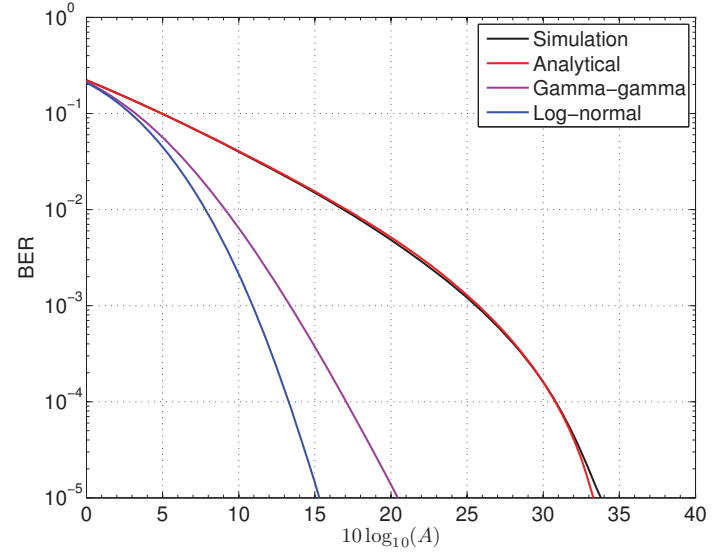

(b) $D=5 \mathrm{~cm}$

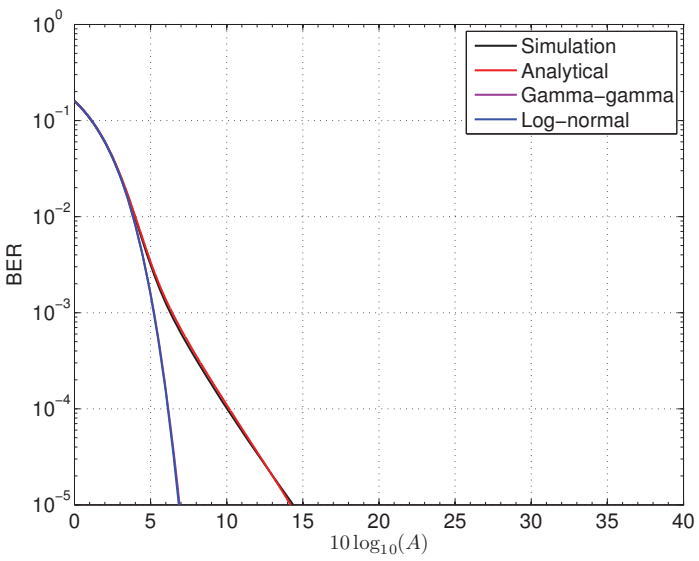

(d) $D=20 \mathrm{~cm}$

Figure 2.8: Comparison for the averaged BER among simulation, analytical method, GG model and LN model. $C_{n}^{2}=5 \times 10^{-15} \mathrm{~m}^{-2 / 3} . L=1.6 \mathrm{Km}$. Gaussian random model. $\sigma_{b}=0.58 \mathrm{~cm} . \sigma_{p}=2.5 \mathrm{~cm} . \sigma_{d}=2.57 \mathrm{~cm} . \Delta x=\Delta y=2.5 \mathrm{~cm}$. $d_{x}=2.5 \mathrm{~mm}$. Coherence radius is $4.2 \mathrm{~cm}$. Rytov variance is 0.24 . $1024 \times 1024$. $0.70 \mathrm{M}$ iterations.

Table 2.5 shows average received beam power $10 \log _{10}(A)$ needed at the receiver to achieve the average $\mathrm{BER}=1 \times 10^{-5}$ estimated by different models with receiver aperture ranges 
Table 2.5: $10 \log _{10}(A)$ requires to achieve $\mathrm{BER}=1 \times 10^{-5}$.

\begin{tabular}{ccccccc}
\hline \multirow{2}{*}{ Model } & \multicolumn{7}{c}{ Aperture $D(\mathrm{~cm})$} \\
\cline { 2 - 7 } & 0 & 5 & 7 & 10 & 14 & 20 \\
\hline Simulation & 39.1 & 33.8 & 33.6 & 31.0 & 25.1 & 14.4 \\
\hline Analytical & 39.1 & 33.3 & 33.3 & 31.0 & 25.0 & 14.2 \\
\hline Gamma-gamma & 27.7 & 20.5 & 17.3 & 13.2 & 9.95 & 6.93 \\
\hline Log-normal & 18.0 & 15.3 & 13.9 & 11.6 & 9.08 & 6.93 \\
\hline
\end{tabular}

from $0 \mathrm{~cm}$ to $20 \mathrm{~cm}$. For point-like $0 \mathrm{~cm}$ receiver aperture, the analytical method estimates almost the same average received beam power $10 \log _{10}(A)$ as obtained by the simulation results. Both of these two methods estimate the $10 \log _{10}(A)$ to be around $39.1 \mathrm{~dB}$ to achieve BER $=10^{-5}$. The required energy estimated by the best fitted GG model is $10 \log _{10}(A)=22.7 \mathrm{~dB}$. It is $11.4 \mathrm{~dB}$ less than the simulated result. In fact, as shown in figure 2.8(a), when $10 \log _{10}(A)=27.7 \mathrm{~dB}$, the actual BER obtained from simulation is $1.6 \times 10^{-3}$. It is 160 times higher than the expected BER. The required energy estimated by the LN model is even smaller than the GG model. By LN model, only $18 \mathrm{~dB}$ average received beam power at receiver is required to achieve $\mathrm{BER}=1 \times 10^{-5}$. For $10 \mathrm{~cm}$ receiver aperture, the required average received beam power $10 \log _{10}(A)$ estimated by simulated fading pdf and the analytical fading pdf are both $31 \mathrm{~dB}$ to achieve $\mathrm{BER}=1 \times 10^{-5}$. The required average received beam power $10 \log _{10}(A)$ estimated by the best fitted GG model is $13.2 \mathrm{~dB}$. It is $17.8 \mathrm{~dB}$ lower and only $42.6 \%$ of that obtained from simulated fading pdf. The required average received beam power $10 \log _{10}(A)$ obtained from LN model is about $11.6 \mathrm{~dB}$, which is $19.4 \mathrm{~dB}$ less than that required by the simulated fading pdf. And the average BER obtained at $10 \log _{10}(A)=13.2 \mathrm{~dB}$ is about $5.3 \times 10^{-3}$. It is about 530 times higher than the required BER. For $20 \mathrm{~cm}$ receiver aperture, both GG model and LN model estimate the system achieves BER $=1 \times 10^{-5}$ when $10 \log _{10}(A)$ is around $6.93 \mathrm{~dB}$. The results from the simulated fading pdf and analytical fading pdf both show the system requires $10 \log _{10}(A)$ to be about $14.2 \mathrm{~dB}$ to achieve BER $=1 \times 10^{-5}$.

The variations of the received optical signal power caused by atmospheric turbulence in 
time are generally of the order of milliseconds [19]. The pointing errors are generally with the vibration frequency less than $100 \mathrm{~Hz}$ [5]. The symbol rates of the FSO communication systems are generally in the order of Mbps or Gbps. Both the turbulence induced fading and the extra pointing error induced fading are slow fading comparing to the data rate. Hence the performance of the FSO communication system is also greatly affected by the outage probability. Table 2.6 shows the probability of $10 \mathrm{~dB}$ outage with the previous $1.6 \mathrm{Km}$ Gaussian extra pointing error channel with $\Delta=2.5 \mathrm{~cm}$ and $\sigma_{p}=2.5 \mathrm{~cm}$ in both $x$ and $y$ directions. The receiver aperture size ranges from point like $0 \mathrm{~cm}$ to $20 \mathrm{~cm}$. For all the studied receiver aperture sizes, the outage probability estimated by the analytical method and the outage probability estimated by the direct wave-optics simulation are almost identical for $10 \mathrm{~dB}$ outage. The outage probability estimated by the best fitted GG model and the LN model are both smaller than that obtained from the simulation and from the analytical method. For the smallest point-like $0 \mathrm{~cm}$ receiver aperture, the outage probability obtained from the simulated fading pdf is $17.8 \%$. The outage probability obtained from the analytical method is $17.6 \%$. The outage probability obtained from the best fitted GG model is $4.86 \%$. And the outage probability obtained from LN fading pdf model is only $10.2 \%$. When the receiver aperture size increases, the outage probabilities estimated by both GG model and LN model quickly decrease. The outage probabilities estimated by the simulated fading pdf and the analytical fading pdf decrease much slower. With $10 \mathrm{~cm}$ receiver aperture, the outage probabilities estimated by both the GG and LN models decrease to zeros. The outage probabilities estimated by both the simulated fading pdf and the analytical fading pdf are both about $3 \%$.

\subsection{Conclusion}

An analytical method is used to calculate the overall fading pdf of the FSO channel with pointing error. The pointing error is the overall displacement between the laser beam weight 
Table 2.6: Probability (\%) for $10 \mathrm{~dB}$ outage. $L=1.6 \mathrm{Km} . C_{n}^{2}=5 \times 10^{-15} \mathrm{~m}^{-2 / 3}$. Gaussian random pointing error model. $\Delta x=\Delta y=2.5 \mathrm{~cm}$, and $\sigma_{p}=2.5 \mathrm{~cm}$ in both $x$ and $y$ directions.

\begin{tabular}{ccccccc}
\hline \multirow{2}{*}{ Model } & \multicolumn{7}{c}{ Aperture $D(\mathrm{~cm})$} \\
\cline { 2 - 7 } & 0 & 5 & 7 & 10 & 14 & 20 \\
\hline Simulation & 17.8 & 10.2 & 7.01 & 3.07 & 0.64 & 0.02 \\
\hline Analytical & 17.6 & 10.4 & 7.08 & 3.15 & 0.66 & 0.02 \\
\hline Gamma-gamma & 4.86 & 1.22 & 0.36 & 0.00 & 0.00 & 0.00 \\
\hline Log-normal & 1.02 & 0.15 & 0.03 & 0.00 & 0.00 & 0.00 \\
\hline
\end{tabular}

center and the receiver aperture center at the receiver plane. Given a FSO channel, the fasttracked beam profile and the fast-tracked on-axis and off-axis fading pdfs are obtained by numerical simulations. With the channel information, the overall fading pdf can be directly calculated using the analytical method with the pointing error model.

Large scale direct wave-optics numerical simulations are performed to verify the calculated fading pdfs. The FSO channels with different propagation distances, turbulence strength, receiver aperture sizes and pointing errors are studied. The simulations cover from weak turbulence regime to strong turbulence regime. Both the fast-tracking residual pointing error model and the beam wander plus transceiver induced extra pointing error model are studied. Three transceiver induced extra pointing error models are studied. They are the Gaussian random model, the uniform random model, and the sine sway model. The analytical method accurately estimates the fading pdf in all our studied cases. The analytical method is also compared with the GG and LN fading pdf models. When only the beam wander is involved, the GG fading pdf model is regarded to be a good estimation of the overall fading pdf if the receiver aperture size is much smaller than the coherence radius. And the LN model is regarded to fit the fading pdf if the receiver aperture size is much larger than the receiver aperture size [38]. However, the analytical method shows a better fit than both the GG and LN fading pdf models from point-like aperture which is much smaller than the coherence radius to the $20 \mathrm{~cm}$ aperture which is much larger than the coherence radius in our studied cases. The analytical method accurately estimates the fading 
pdfs in all the studied pointing errors. Both the GG model and the LN model underestimate the average BER of the FSO communication system. The outage probability is also underestimated by both the GG model and the LN model. Due to the long deep fading period of the slow fading channel, this will largely underestimate the damage caused by outage to the system. 


\section{Chapter 3}

\section{Fading Pdf with Beam Diverging Angle}

\subsection{Introduction}

In a FSO communication system without perfect fast-tracking, the laser beam spot randomly moves on the receiver aperture plane due to the pointing error. When the laser beam moves away from the receiver aperture, deep fading or even outage may occur. To avoid the serious system performance degradation caused by the deep fading and even outage, one of the methods is to change the diverging angle of the transmitted laser beam.

When the beam diverging angle increases, the laser spot size at the receiver plane becomes larger. With a larger beam spot size, suppose under the same pointing error, the chance that the large part of the laser beam moves out of the receiver aperture becomes smaller. However, when the beam spot size is larger than the receiver aperture size, an larger beam diverging angle generally causes smaller portion of the laser beam power to be received on average by the receiver aperture. This causes a larger average received power loss compared to the smaller beam diverging angle case. The large average received power loss also 
seriously degrades the system performance. An optimal beam diverging angle that balances the deep fading and the average power loss given the receiver aperture size, propagation distance and the atmospheric turbulence condition is discussed in [1]. It is found that when the receiver aperture size is large comparing to the beam spot size, the FSO communication system with focused beam can achieve better performance. When the receive aperture size is smaller than the beam spot size, large beam diverging angle is preferred. With the optimal beam diverging angle, better system performance can be achieved with the same transmitter power comparing to FSO communication system with the collimated beam case. In the past, most studies focused on the FSO communication system with collimated laser beam. And to our best knowledge, there is no general mathematical model on the fading pdf of the FSO communication system with beam diverging angle.

In this chapter, the analytical method is used to analysis the overall fading pdf of the FSO communication system with a point-like receiver aperture and a beam diverging angle. As mentioned in the chapter 2 , in the analytical method, the overall fading pdf is calculated from the three factors, namely, the fast-tracked on-axis and off-axis fading pdfs, the fasttracked beam profile, and the pointing error. The impact of beam diverging angle on the these factors are studied.

Large-scale wave-optics numerical simulations prove that for point-like receiver aperture, the fast-tracked fading pdfs can still be well modeled by the gamma pdf model at both on-axis and off-axis locations when the laser beam is transmitted with either focused or diverged beam angle. Based on the gamma pdf model, only variance information is required to obtain the fast-tracked on-axis and off-axis fading pdfs. It is found that when the beam diverging angle increases, variance of the normalized fast-tracked on-axis and off-axis received beam power fading also stretches to the off-axis locations. That is to say, the variance of the fast-tracked fading changes slower and the changes in a wider range as the sample location move away from the fast-tracked beam center. The numerical simulations show that fast-tracked beam profile also grows wider as the beam diverging angle 
increases. For both focused and diverged beam cases, the radius of the fast-tracked beam profile is close to that of the diffraction pattern. It is a little larger due to the turbulence induced beam broadening effect [11]. In this chapter, the pointing error is the turbulence induced beam wander. When the laser beam is transmitted with a beam diverging angle, the beam wander still follows the two dimensional Gaussian distribution [11]. The numerical simulation shows that the variance of the turbulence induced beam wander decreases as the beam diverging angle increases. As the possible extra pointing error induced by the relative mechanical movement of the transmitter and receiver can be considered to be independent of the beam diverging angle, the variance of the overall pointing error is also expected to decrease as the beam diverging angle increases for the general pointing error case. Hence It is possible that for a FSO communication system with a small beam diverging angle, such as with the focused beam case, the variance of the normalized fast-tracked on-axis and off-axis fading changes in the whole pointing error range. In this case, both the fast-tracked on-axis and off-axis fading contributes to the overall fading pdf. For a FSO communication system with a quite large beam diverging angle, the variance of normalized fast-tracked on-axis and off-axis fading keeps almost unchanged in most of pointing error range. The fast-tracked on-axis and off-axis fadings can be represented by the fast-tracked on-axis fading. As the fast-tracked beam profile also changes little during the pointing error range in this well diverged beam case, the overall fading pdf can be approximated by the fast-tracked on-axis fading pdf.

In this chapter, direct large-scale numerical wave-optics simulations are performed to study the channel informations needed by the analytical method to calculate the overall fading pdf when the laser beam is propagated with a beam diverging angle. The studied channel informations are, namely, the fast-tracked beam profile, the fast-tracked on-aixs and offaxis fading pdfs. The Kolmogorov phase screens are used to simulate the atmospheric turbulence. In the FSO system, the Gaussian laser beam is assumed to propagation along a horizontal direction. The laser wavelength is $\lambda=1.54 \times 10^{-6} \mathrm{~m}$. The direct numerical wave-optics simulations are performed under different turbulence strengths, propagation 
distances and beam diverging angles. The refractive index structure parameter ranges from $5 \times 10^{-15} \mathrm{~m}^{-2 / 3}$ to $5 \times 10^{-14} \mathrm{~m}^{-2 / 3}$. The propagation distance $L$ ranges from $0.75 \mathrm{Km}$ to $2.5 \mathrm{Km}$ in the focused beam cases. And $L$ ranges from $0.5 \mathrm{Km}$ to $2 \mathrm{Km}$ in the diverged beam cases. The receiver aperture is the point-like $0 \mathrm{~cm}$ aperture, which contains only single pixel in the simulation field. It is much smaller than the coherence radius and can be regarded to be a point in the optical field. The pointing error is the beam wander induced pointing error. To accurately obtain especially the deep fading tail of the fading pdf, the simulations are conducted under large grid size with high resolution. The field grid size ranges from $1024 \times 1024$ to $2048 \times 2048$. The field resolution ranges from $0.625 \mathrm{~mm}$ to $2.5 \mathrm{~mm}$, which is much smaller than the coherence radius in each case. The iteration number ranges from 0.19 million to 0.62 million to obtain a smooth pdf curve and enough fading pdf tail information.

The overall fading pdf curves calculated by the analytical method accurately match the corresponding fading pdf curves obtained from numerical wave-optics simulations in all the studied cases with both focused and diverged laser beam. As the diverging angle changes, the fast-tracked beam profile size changes. When the fast-tracked beam profile size is at the range of the turbulence induced beam wander, the overall fading pdf is greatly affected by the beam wander induced fading. The analytical method has better fit than the best fitted gamma-gamma (GG) and the log-normal (LN) fading pdf models. When the beam is well diverged, the fast-tracked beam profile size is much larger than the beam wander range. And the fast-tracked off-axis fading pdf changes much slower as the receiver aperture location departs from the fast-tracked beam center. The overall fading pdf is close to the fast-tracked on-axis fading pdf.

The rest of this chapter is organized as follows. Section 3.2 describes the direct waveoptics simulation setup for the FSO communication system with beam diverging angle. In section 3.3, first, the fast-tracked on-axis and off-axis fading pdfs are proved to be well modeled by the gamma pdf model with both focused and diverged laser beam. The an- 
alytically calculated fading pdfs are then verified by the directly simulated fading pdfs. Conclusions are given in section 3.4.

\subsection{Simulation Setup}

Large-scale wave-optics numerical simulations are conducted for FSO communication system with point-like receiver aperture in this chapter to provide two sets of information. First, numerical simulations provide the channel information needed for the analytical method to calculated the overall fading pdf in each optical channel. They are namely, fast-tracked on-axis and off-axis fading pdfs, fast-tracked beam profile, and pointing error. The pointing error in this chapter is the turbulence induced beam wander. It is known that fast-tracked on-axis and off-axis fading pdfs can be well modeled by the gamma pdf model for the FSO communication system with point-like receiver when a collimated laser beam is transmitted [57]. Large-scale numerical simulations are used in this chapter to prove when the laser beam is transmitted with a diverging angle, the fast-tracked on-axis and off-axis fading pdfs received by the point-like receiver still can be well formulated by the gamma pdf model. With the gamma pdf model, only fading variance at on-axis and off-axis locations are required to be obtained from numerical simulation to generate the fast-tracked on-axis and off-axis fading pdfs. The turbulence induced beam wander is shown to follow the two dimensional Gaussian distribution [11]. For collimated laser beam, the standard deviation can be calculated from 2.28. When the laser beam is transmitted with an diverging angle, the standard deviation is directly obtained from the numerical simulation. The fast-tracked beam profile is also directly obtained from the simulation. With the fasttracked on-axis and off-axis fading pdfs, the fast-tracked beam profile, and the turbulence induced beam wander, the overall fading pdfs of the received beam power are calculated using the analytical method for both focused and diverged laser beams. Then the overall fading pdfs are also obtained from direct numerical simulation to verify the analytically 
calculated overall fading pdfs.

The large-scale numerical wave-optics simulations are conducted under different atmospheric turbulence strengths, and propagation distances for both focused and diverged laser beam cases. The simulation program is verified by both analytical analysis and the Matlab AO toolbox. In the simulations, the Gaussian laser beam propagates along a horizontal direction. The Gaussian laser beam radius is $5 \mathrm{~cm}$ at the transmitter plane. The laser wavelength is $\lambda=1.54 \times 10^{-6} \mathrm{~m}$. The aerosol scattering effects [29], [30] caused by haze, rain, snow, and fog are not considered. The transmitter and receiver are assumed to be constantly perfectly aimed. Hence mechanical displacement induced extra pointing error is not considered. The atmospheric turbulence is simulated by 10 phase screens uniformly placed between the transmitter and the receiver apertures. Table 3.1 and table 3.2 show the parameters for focused beam case and diverged beam case, respectively. The propagation distance $L$ ranges from $0.75 \mathrm{Km}$ to $2.5 \mathrm{Km}$ for focused beam case. $L$ ranges from $0.5 \mathrm{Km}$ to $2 \mathrm{Km}$ for diverged beam case. For focused beam case, the laser beam is exactly focused at the receiver aperture center and $L / F=1$, where $F$ is the focal length. For diverged case, the laser beam is reversely focused with $F=-L$. Hence $L / F=-1$ for all the diverged beam cases. The Kolmogorov spectrum is used to generate the phase screens. The grid size of the phase screen $N \times N$ ranges from $1024 \times 1024$ to $2048 \times 2048$. The resolution $d x$ of the phase screen ranges from $0.625 \mathrm{~mm}$ to $2.5 \mathrm{~mm}$. The resolution $d x$ is much smaller than the corresponding coherence radius $r_{0}$ and satisfies the restrictions to accurately simulate the wave propagation. The grid size $N \times N$ is large enough to avoid the alias effect with the given grid size. During the simulation, the laser beam is assumed to be received by the point-like $0 \mathrm{~cm}$ receiver. The point-like receiver contains only single pixel in the receiver plane. The radius $\tilde{r}$ of the equivalent circular aperture [38] are also listed in table 3.1 and table 3.2 for focused and diverged beam cases, respectively. The point-like receiver is much smaller than the coherence radius. It can be regarded to a point in the optical field. 
Table 3.1: Simulation parameters for focused beam. $L / F=1$.

\begin{tabular}{ccccccc}
\hline $\begin{array}{c}L \\
(\mathrm{Km})\end{array}$ & $\begin{array}{c}C_{n}^{2} \\
\left(\mathrm{~m}^{-2 / 3}\right)\end{array}$ & $N$ & $\begin{array}{c}r_{0} \\
(\mathrm{~mm})\end{array}$ & $\begin{array}{c}d x \\
(\mathrm{~mm})\end{array}$ & $\begin{array}{c}M \\
\left(\times 10^{6}\right)\end{array}$ & $\begin{array}{c}\tilde{r} \\
(\mathrm{~mm})\end{array}$ \\
\hline 0.75 & $5 \times 10^{-14}$ & 2048 & 16.8 & 0.5 & 0.19 & 0.28 \\
\hline 1 & $5 \times 10^{-15}$ & 1024 & 56.1 & 1.25 & 0.32 & 0.71 \\
\hline 2 & $5 \times 10^{-15}$ & 1536 & 37.0 & 1.4 & 0.48 & 0.79 \\
\hline 2.5 & $5 \times 10^{-15}$ & 1536 & 32.4 & 1.5 & 0.62 & 0.99 \\
\hline
\end{tabular}

Table 3.2: Simulation parameters for diverged beam. $L / F=-1$.

\begin{tabular}{ccccccc}
\hline $\begin{array}{c}L \\
(\mathrm{Km})\end{array}$ & $\begin{array}{c}C_{n}^{2} \\
\left(\mathrm{~m}^{-2 / 3}\right)\end{array}$ & $N$ & $\begin{array}{c}r_{0} \\
(\mathrm{~mm})\end{array}$ & $\begin{array}{c}d x \\
(\mathrm{~mm})\end{array}$ & $\begin{array}{c}M \\
\left(\times 10^{6}\right)\end{array}$ & $\begin{array}{c}\tilde{r} \\
(\mathrm{~mm})\end{array}$ \\
\hline 0.5 & $5 \times 10^{-14}$ & 2048 & 21.4 & 0.65 & 0.26 & 0.37 \\
\hline 0.5 & $5 \times 10^{-15}$ & 1024 & 85.1 & 0.9 & 0.32 & 0.51 \\
\hline 1 & $5 \times 10^{-15}$ & 1024 & 56.1 & 1.25 & 0.47 & 0.71 \\
\hline 2 & $5 \times 10^{-15}$ & 1536 & 37.0 & 1.4 & 0.30 & 0.79 \\
\hline
\end{tabular}

\subsection{Simulation Results}

\subsubsection{Fast-tracked Fading Pdf}

With the perfect fast-tracking, the overall pointing error is assumed to be well compensated [21], [27], [47]. The received beam power vibrates due to the turbulence induced wave front distortion.

For FSO communication system with collimated beam, the fast-tracked on-axis and offaxis fading pdfs can be modeled by the gamma pdf model [57]. Large scale numerical simulations in this chapter prove when the laser beam is transmitted with a diverging angle, the fast-tracked on-axis and off-axis fading pdfs can also be closely modeled by the gamma pdf model. Figure 3.1(a), 3.1(b), 3.1(c), and 3.1(d) show the gamma fit of the fast-tracked fading pdfs obtained by point-like receiver at on-axis and off-axis locations for Gaussian focused beam with $L / F=1$ at $L=1 \mathrm{Km}, L=2 \mathrm{Km}$, and $L=2.5 \mathrm{Km}$ with $C_{n}^{2}=$ $5 \times 10^{-15} \mathrm{~m}^{-2 / 3}$ and $L=0.75 \mathrm{Km}$ with $C_{n}^{2}=5 \times 10^{-14} \mathrm{~m}^{-2 / 3}$. Figure 3.2(a), 3.2(b), 3.2(c), 
and 3.2(d) show the gamma fit of the fast-tracked fading pdfs obtained with point-like receiver at both on-axis and off-axis locations for Gaussian diverged beam with $L / F=-1$ at $L=0.5 \mathrm{Km}, L=1 \mathrm{Km}$, and $L=2 \mathrm{Km}$ with $C_{n}^{2}=5 \times 10^{-15} \mathrm{~m}^{-2 / 3}$ and $L=0.5 \mathrm{Km}$ with

$C_{n}^{2}=5 \times 10^{-14} \mathrm{~m}^{-2 / 3}$. The pdfs are shown on the $10 \log _{10}(h)$ scale where $h$ is the normalized channel state to emphasis the deep fading tails of the fading pdfs. The gamma fading pdfs are generated by the variance of the simulated fast-tracked on-axis and off-axis normalized power fading. It shows that the simulated fast-tracked fading pdfs follow the gamma pdf model quite well at both an-axis and off-axis locations in all the studied beam diverging angles, propagation distances, and turbulence strengths. In the rest of this chapter, the fast-tracked on-axis and off-axis fading pdfs are generated using their corresponding variances based on the gamma pdf model.

\subsubsection{Overall Fading Pdf}

The analytical method considered three factors to calculate the overall fading pdf with beam wander. They are, namely, fast-tracked beam profile, fast-tracked on-axis and offaxis fading pdfs, and the beam wander.

Figure 3.3(a), 3.3(b), 3.3(c), 3.3(d) and figure 3.4(a), 3.4(b), 3.4(c), 3.4(d) show the fasttracked beam profile for both focused and diverged beams at different turbulence strengths and propagation distances. The $x$ axis is the distance $r$ to the fast-tracked beam center. The maximum beam intensity of the Gaussian laser beam at the transmitter plane is assumed to be 1 .

The beam radius $W$ (place with $1 / e^{2}$ of the maximum beam intensity) of the fast-tracked beam profile is listed in table 3.3 and table 3.4 for focused and diverged beam, respectively. The beam radius of the corresponding diffraction pattern $\tilde{W}$ are also listed for comparison. The radius of the fast-tracked beam profile $W$ is close to the radius of the diffraction pattern 


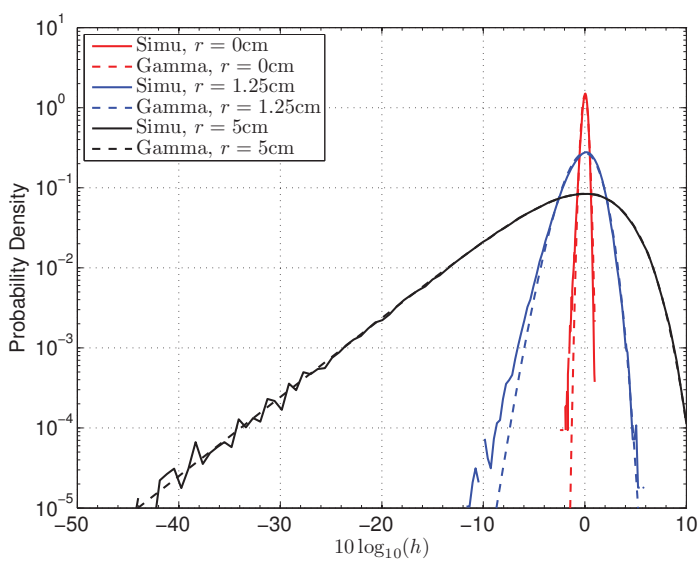

(a) $L=1 \mathrm{Km}, C_{n}^{2}=5 \times 10^{-15} \mathrm{~m}^{-2 / 3}$.

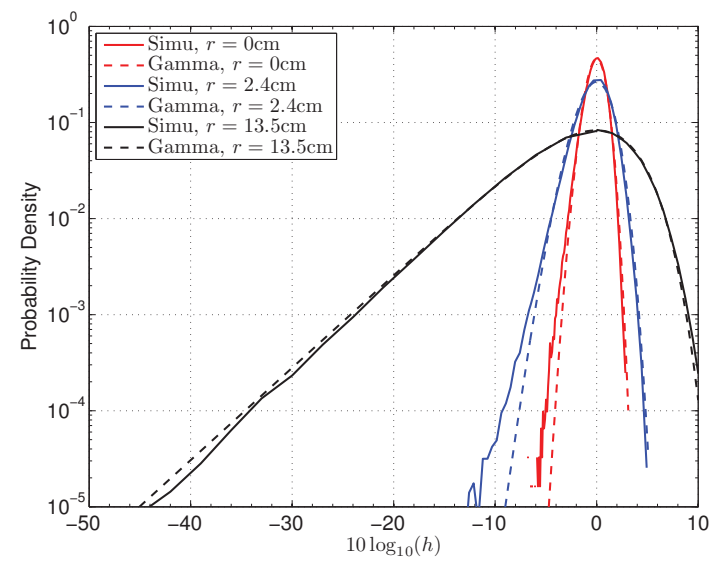

(c) $L=2.5 \mathrm{Km}, C_{n}^{2}=5 \times 10^{-15} \mathrm{~m}^{-2 / 3}$.

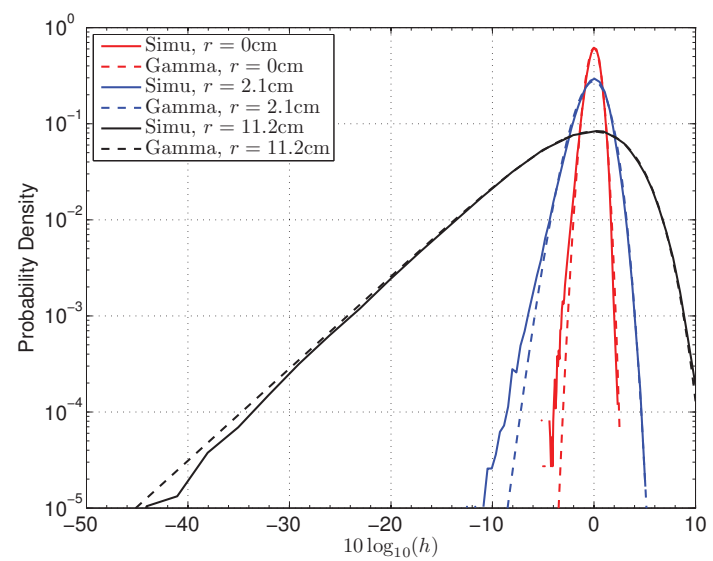

(b) $L=2 \mathrm{Km}, C_{n}^{2}=5 \times 10^{-15} \mathrm{~m}^{-2 / 3}$.

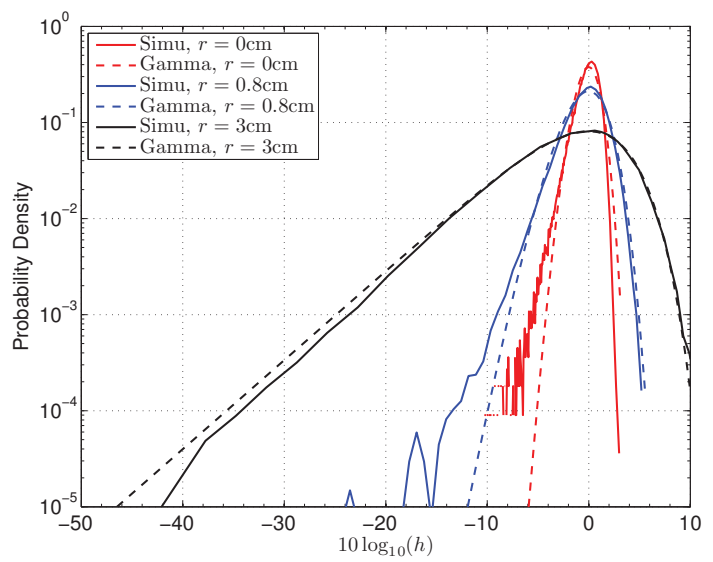

(d) $L=0.75 \mathrm{Km}, C_{n}^{2}=5 \times 10^{-14} \mathrm{~m}^{-2 / 3}$.

Figure 3.1: Fast-tracked on-axis and off-axis fading pdfs for focused beam.

$\tilde{W}$. The atmospheric turbulence increases the radius a little due to the beam broadening effect.

For the Gaussian beam with diverging angle, the turbulence induced beam wander still follows the two dimensional independent Gaussian distribution. The standard deviation $\sigma_{w}$ of the beam wander for focused beam and diverged beam is listed in table 3.3 and table 3.4, respectively. And the beam wander standard deviation $\tilde{\sigma_{w}}$ of the corresponding collimated beam is also listed for comparison. In our studied cases, as the beam diverging angle increases, the standard deviation of beam wander $\sigma_{w}$ slightly decreases. This corresponds 


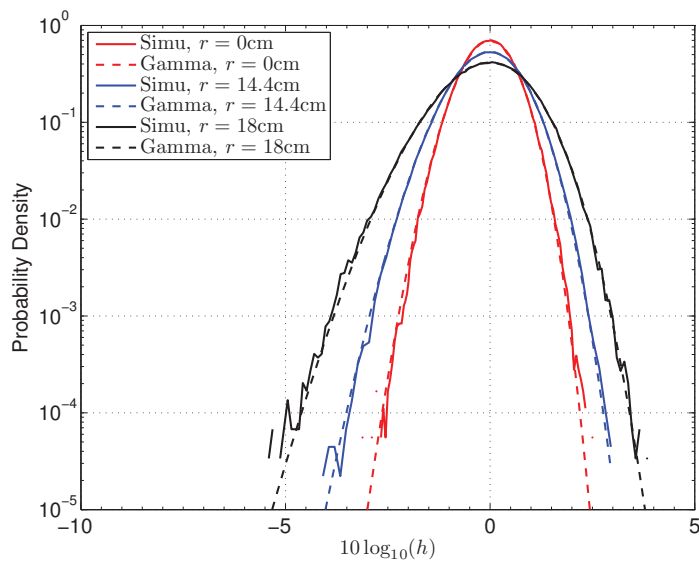

(a) $L=500 \mathrm{~m}, C_{n}^{2}=5 \times 10^{-15} \mathrm{~m}^{-2 / 3}$.

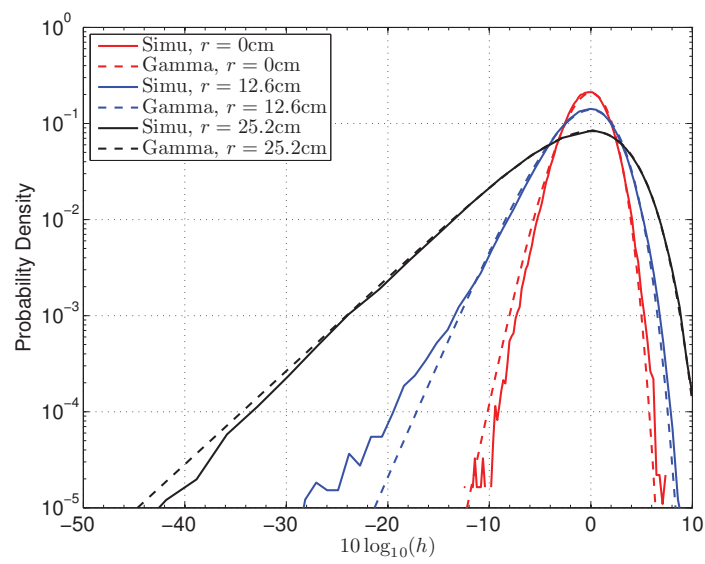

(c) $L=2 \mathrm{Km}, C_{n}^{2}=5 \times 10^{-15} \mathrm{~m}^{-2 / 3}$.

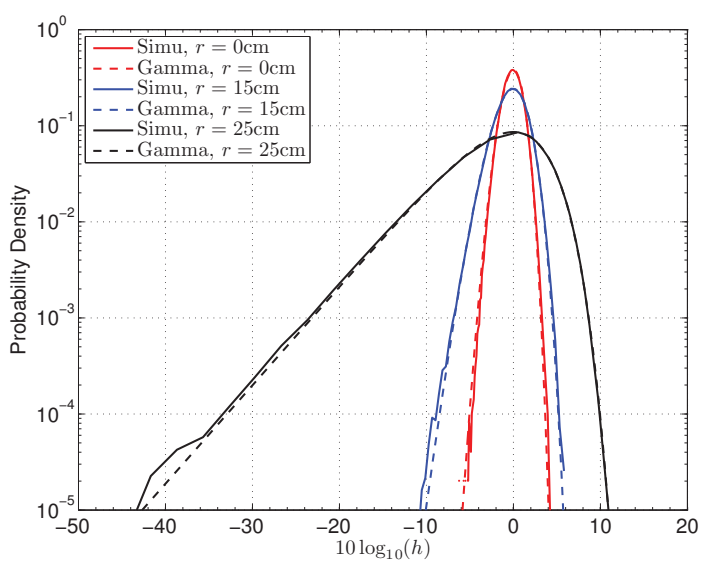

(b) $L=1 \mathrm{Km}, C_{n}^{2}=5 \times 10^{-15} \mathrm{~m}^{-2 / 3}$.

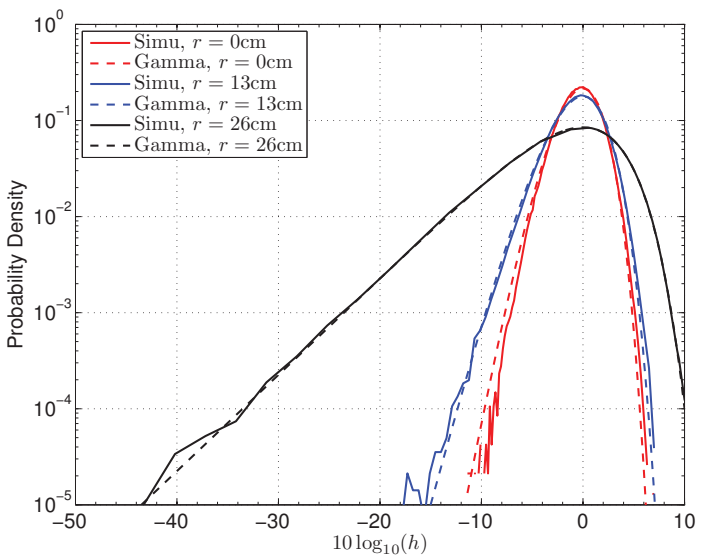

(d) $L=500 \mathrm{~m}, C_{n}^{2}=5 \times 10^{-14} \mathrm{~m}^{-2 / 3}$.

Figure 3.2: Fast-tracked on-axis and off-axis fading pdfs for diverged beam.

to a small revise to $k$ obtained in the collimated beam case in 2.28.

As the fast-tracked on-axis and off-axis fading pdfs can be well modeled by the gamma fading pdf model, only variance information is required to be obtained through numerical simulation. Figure 3.3(a), 3.3(b), 3.3(c), and 3.3(d) show the standard deviation of the normalized fast-tracked fading obtained with point-like receiver at both on-axis and off-axis locations for Gaussian focused beam with $L / F=1$ at $L=1 \mathrm{Km}, L=2 \mathrm{Km}$ and $L=2.5 \mathrm{Km}$ with $C_{n}^{2}=5 \times 10^{-15} \mathrm{~m}^{-2 / 3}$ and $L=0.75 \mathrm{Km}$ with $C_{n}^{2}=5 \times 10^{-14} \mathrm{~m}^{-2 / 3}$. Figure 3.4(a), 3.4(b), 3.4(c), and 3.4(d) show the standard deviation of the normalized fasttracked fading obtained with point-like receiver at both on-axis and off-axis locations for 
Table 3.3: Beam radius $W$ and beam wander standard deviation $\sigma_{w}$ for focused beam. $L / F=1$.

\begin{tabular}{cccccc}
\hline $\begin{array}{c}\mathrm{L} \\
(\mathrm{Km})\end{array}$ & $\begin{array}{c}C_{n}^{2} \\
\left(\mathrm{~m}^{-2 / 3}\right)\end{array}$ & $\begin{array}{c}W \\
(\mathrm{~cm})\end{array}$ & $\begin{array}{c}\tilde{W} \\
(\mathrm{~cm})\end{array}$ & $\begin{array}{c}\sigma_{w} \\
(\mathrm{~cm})\end{array}$ & $\begin{array}{c}\tilde{\sigma}_{w} \\
(\mathrm{~cm})\end{array}$ \\
\hline 0.75 & $5 \times 10^{-14}$ & 1.22 & 0.74 & 0.87 & 0.59 \\
\hline 1 & $5 \times 10^{-15}$ & 1.01 & 0.98 & 0.31 & 0.29 \\
\hline 2 & $5 \times 10^{-15}$ & 2.06 & 1.96 & 0.87 & 0.81 \\
\hline 2.5 & $5 \times 10^{-15}$ & 2.59 & 2.45 & 1.20 & 1.13 \\
\hline
\end{tabular}

Table 3.4: Beam radius $W$ and beam wander standard deviation $\sigma_{w}$ for diverged beam. $L / F=-1$.

\begin{tabular}{cccccc}
\hline $\begin{array}{c}\mathrm{L} \\
(\mathrm{Km})\end{array}$ & $\begin{array}{c}C_{n}^{2} \\
\left(\mathrm{~m}^{-2 / 3}\right)\end{array}$ & $\begin{array}{c}W \\
(\mathrm{~cm})\end{array}$ & $\begin{array}{c}\tilde{W} \\
(\mathrm{~cm})\end{array}$ & $\begin{array}{c}\sigma_{w} \\
(\mathrm{~cm})\end{array}$ & $\begin{array}{c}\tilde{\sigma}_{w} \\
(\mathrm{~cm})\end{array}$ \\
\hline 0.5 & $5 \times 10^{-14}$ & 10.05 & 10.01 & 0.31 & 0.32 \\
\hline 0.5 & $5 \times 10^{-15}$ & 10.02 & 10.01 & 0.10 & 0.10 \\
\hline 1 & $5 \times 10^{-15}$ & 10.07 & 10.05 & 0.27 & 0.29 \\
\hline 2 & $5 \times 10^{-15}$ & 10.30 & 10.20 & 0.78 & 0.81 \\
\hline
\end{tabular}

Gaussian diverged beam with $L / F=-1$ at $L=0.5 \mathrm{Km}, L=1 \mathrm{Km}$ and $L=2 \mathrm{Km}$ with $C_{n}^{2}=5 \times 10^{-15} \mathrm{~m}^{-2 / 3}$ and $L=0.5 \mathrm{Km}$ with $C_{n}^{2}=5 \times 10^{-14} \mathrm{~m}^{-2 / 3}$. The vertical line shows the $3 \sigma_{w}$ region of the beam wander. With the fast-tracked fading variance, the fast-tracked on-axis and off-axis fading pdfs are generated based on the gamma pdf model.

For focused beams, the fast-tracked fading standard deviation $\sigma_{t}$ changes quickly as the point-like receiver aperture moves away from the fast-tracked beam center. For $C_{n}^{2}=5 \times$ $10^{-15} \mathrm{~m}^{-2 / 3}$, at $L=1 \mathrm{Km}$, the standard deviation $\sigma_{t}$ begins to rapidly increase as soon as the receiver aperture moves apart from the fast-tracked beam center. $\sigma_{t}$ saturates soon when the receiver aperture is about $3 \mathrm{~cm}$ away from the fast-tracked beam center. As the turbulence induced beam wander standard deviation $\sigma_{w}$ is $0.31 \mathrm{~cm}$, the fast-tracked fading standard deviation $\sigma_{t}$ keeps changing in the whole $3 \sigma_{w}$ beam wander region. When $L$ increases to $2.5 \mathrm{Km}$, the fast-tracked fading standard deviation $\sigma_{t}$ begins to rapidly increases when the receiver aperture is about $2 \mathrm{~cm}$ away from the fast-tracked beam center. $\sigma_{t}$ begins to saturate when the receiver aperture is about $5 \mathrm{~cm}$ away from the fast-tracked beam center. 
The beam wander standard deviation $\sigma_{w}$ is $1.2 \mathrm{~cm}$ in this turbulence channel. Hence the $\sigma_{t}$ also keeps changing in the whole $3 \sigma_{w}$ beam wander region. For $C_{n}^{2}=5 \times 10^{-15} \mathrm{~m}^{-2 / 3}$, at $L=0.75 \mathrm{Km}, \sigma_{w}$ is $0.87 \mathrm{~cm}$ and $\sigma_{t}$ saturates after the receiver aperture is about $2 \mathrm{~cm}$ away from the receiver aperture. In the $3 \sigma_{w}$ region the $\sigma_{t}$ also keeps changing.

For diverged beam, the beam spot size is much larger. The standard deviation $\sigma_{t}$ keeps almost unchanged around the fast-tracked center compared to the focused beam case. For $C_{n}^{2}=5 \times 10^{-15} \mathrm{~m}^{-2 / 3}$, at $L=1 \mathrm{Km}, \sigma_{t}$ almost keeps the same until the receiver aperture is about $10 \mathrm{~cm}$ away from the fast-tracked beam center. For comparison, in the focused beam case, the $\sigma_{t}$ changes immediately as the receiver aperture moves apart from the fast-tracked beam center. And the $\sigma_{t}$ of this diverged beam begins to saturate until the receiver aperture is about $25 \mathrm{~cm}$ away from the fast-tracked beam center, which is also greatly increased comparing to the focused beam case. On the other hand, the turbulence induced beam wander standard deviation $\sigma_{w}$ decreases a little from $0.31 \mathrm{~cm}$ of the focused beam to $0.27 \mathrm{~cm}$ of the diverged beam. The $\sigma_{t}$ keeps unchanged in the $3 \sigma_{w}$ beam wander region. At $L=$ $2 \mathrm{Km}$, the $\sigma_{t}$ keeps almost unchanged until the receiver aperture is more than $5 \mathrm{~cm}$ away from the fast-tracked beam center, while in the focused beam case, the $\sigma_{t}$ begins to rapidly increase as the aperture is $1 \mathrm{~cm}$ away from fast-tracked beam center. The beam wander standard deviation $\sigma_{w}$ decreases from $0.87 \mathrm{~cm}$ to $0.78 \mathrm{~cm}$ comparing to the focused beam case in the same turbulence channel. Hence $\sigma_{t}$ keeps the same in the $3 \sigma_{w}$ beam wander region. This is also valid in other studied diverged beam cases.

Based on the fast-tracked on-axis and off-axis fading pdfs, the two dimensional independent Gaussian beam wander model, and the fast-tracked beam profile, the overall fading pdfs of received laser beam power can be calculated with the analytical method by 2.10 .

Figure 3.5(a), 3.5(b), 3.5(c), and 3.5(d) show the calculated overall fading pdfs of the normalized beam power received by point-like receiver through atmospheric turbulence channel for Gaussian focused beam with $L / F=1$ at $L=1 \mathrm{Km}, L=2 \mathrm{Km}$ and $L=2.5 \mathrm{Km}$ with 


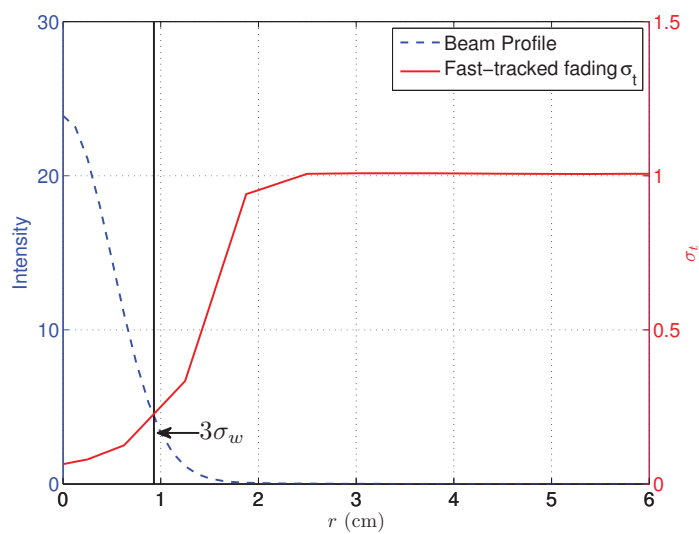

(a) $L=1 \mathrm{Km}, C_{n}^{2}=5 \times 10^{-15} \mathrm{~m}^{-2 / 3}$.

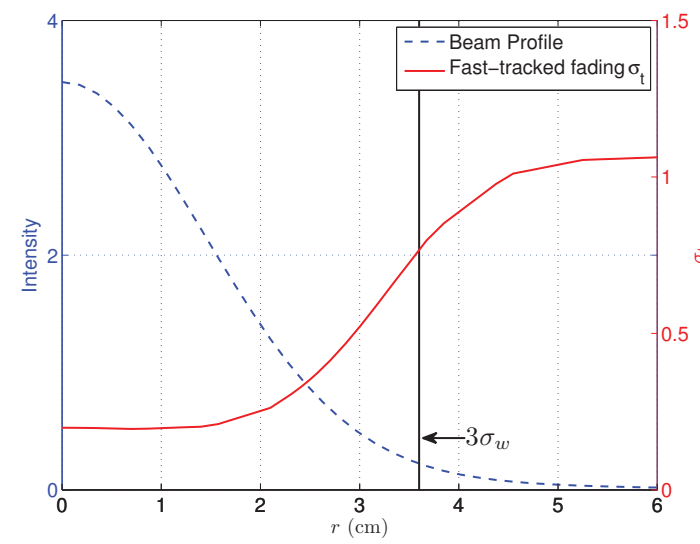

(c) $L=2.5 \mathrm{Km}, C_{n}^{2}=5 \times 10^{-15} \mathrm{~m}^{-2 / 3}$.

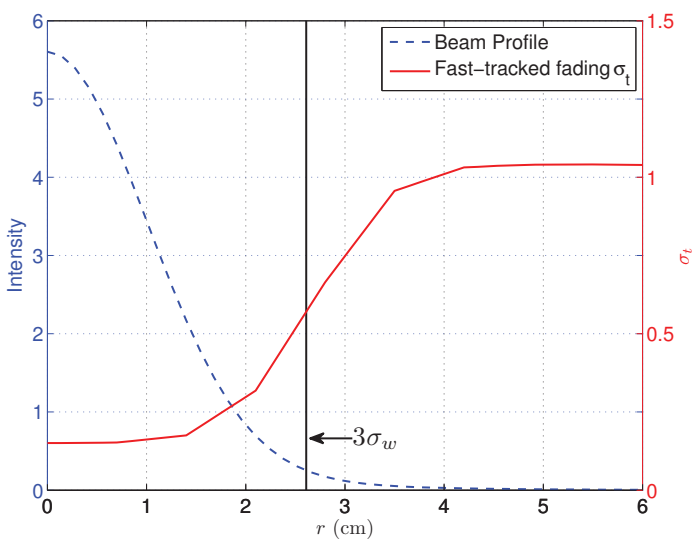

(b) $L=2 \mathrm{Km}, C_{n}^{2}=5 \times 10^{-15} \mathrm{~m}^{-2 / 3}$.

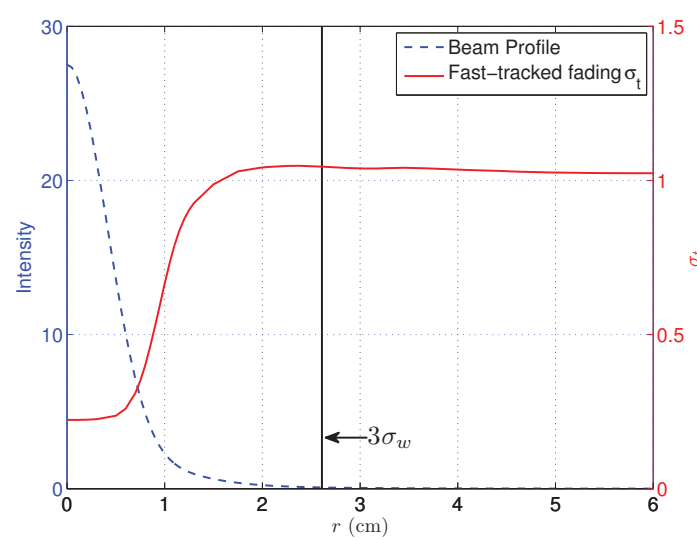

(d) $L=0.75 \mathrm{Km}, C_{n}^{2}=5 \times 10^{-14} \mathrm{~m}^{-2 / 3}$.

Figure 3.3: Fast-tracked beam profile and fading variance for focused beam.

$C_{n}^{2}=5 \times 10^{-15} \mathrm{~m}^{-2 / 3}$ and $L=0.75 \mathrm{Km}$ with $C_{n}^{2}=5 \times 10^{-14} \mathrm{~m}^{-2 / 3}$. The calculated overall fading pdfs are compared with the overall fading pdfs obtained from direct wave-optics numerical simulations. The analytically calculated overall fading pdfs accurately fit the directly simulated overall fading pdfs in all the studied focused beam cases even when the fading is as deep as $-40 \mathrm{~dB}$ and the probability density is as low as $10^{-4}$. For comparison, the analytically calculated overall fading pdfs using only the fast-tracked on-axis fading pdf is also shown. In this on-axis only model, the fast-tracked on-axis fading pdf $P_{t_{-} o}\left(\frac{I_{\text {overall }}}{I_{\text {displace }}}\right)$ is used to replace $P_{t}\left(\frac{I_{\text {overall }}}{I_{\text {displace }}} \mid I_{\text {displace }}\right)$ in 2.10 . It is equivalent to regard the fast-tracked normalized fading follows the fast-tracked on-axis fading pdf at both on-axis and off-axis locations. As the fast-tracked fading pdf changes in the beam wander range in 


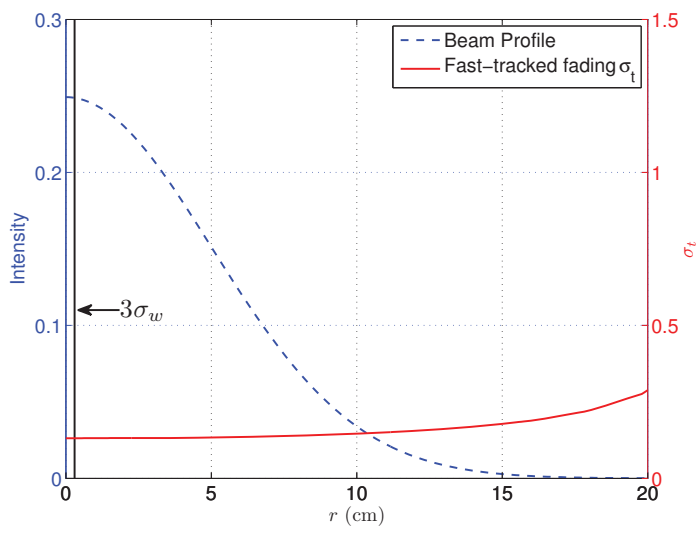

(a) $L=500 \mathrm{~m}, C_{n}^{2}=5 \times 10^{-15} \mathrm{~m}^{-2 / 3}$.

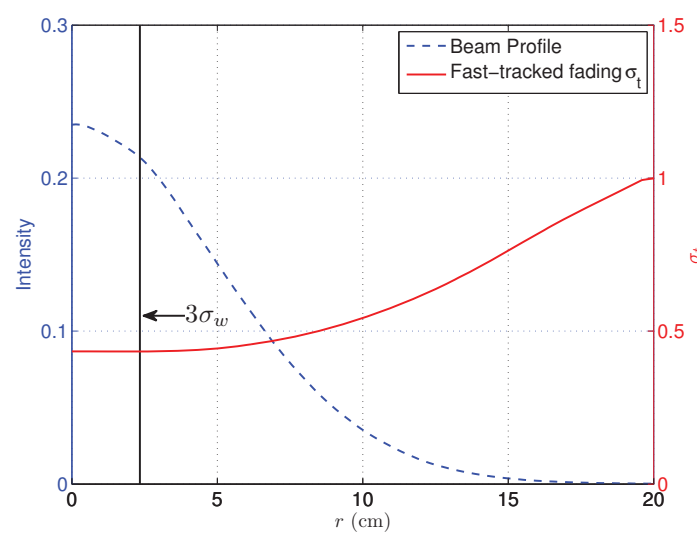

(c) $L=2 \mathrm{Km}, C_{n}^{2}=5 \times 10^{-15} \mathrm{~m}^{-2 / 3}$.

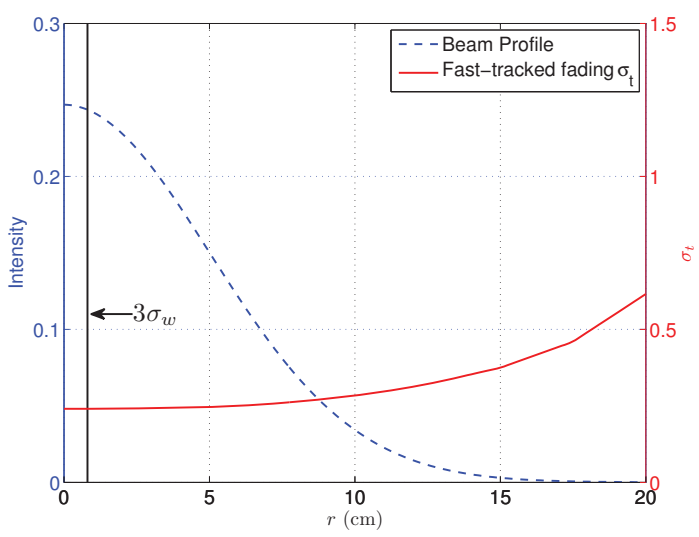

(b) $L=1 \mathrm{Km}, C_{n}^{2}=5 \times 10^{-15} \mathrm{~m}^{-2 / 3}$.

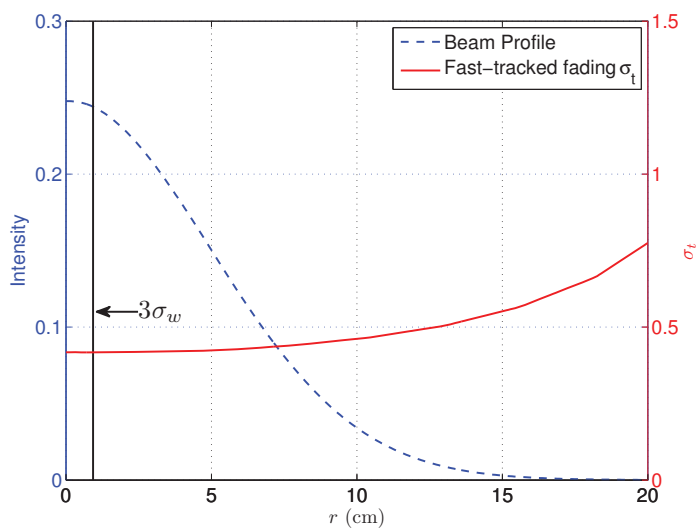

(d) $L=500 \mathrm{~m}, C_{n}^{2}=5 \times 10^{-14} \mathrm{~m}^{-2 / 3}$.

Figure 3.4: Fast-tracked beam profile and fading variance for diverged beam.

the focused beam case, the fading pdf calculated with only the fast-tracked on-axis fading pdf generally deviates from the directly simulated fading pdf and also the overall fading pdf calculated with both on-axis and off-axis fast-tracked fading pdfs. Comparing figure 3.5(a) with figure 3.5(b), 3.5(c), and 3.5(d), it is shown that the greater the fast-tracked fading pdf changes in the beam wander range, the more the fading pdf calculated with on-axis only fast-tracked fading pdf deviates from the simulated fading pdf. The overall fading pdfs are also compared with the log-normal (LN) and the best fitted gamma-gamma (GG) fading pdf models. The LN fading pdf is generated according to the variance of the simulated normalized beam power fading. The best fitted GG fading pdf selects the $\alpha$ and $\beta$ parameters to have the closest fit to the simulated overall fading pdf while maintaining the same 


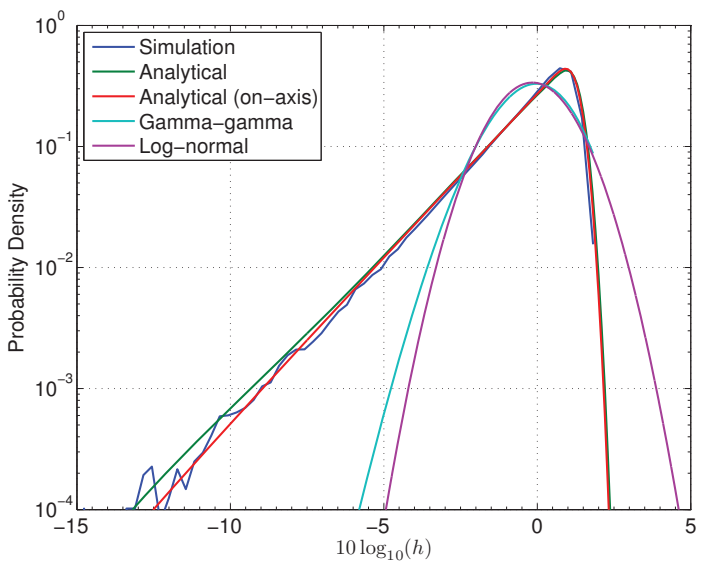

(a) $L=1 \mathrm{Km}, C_{n}^{2}=5 \times 10^{-15} \mathrm{~m}^{-2 / 3}$.

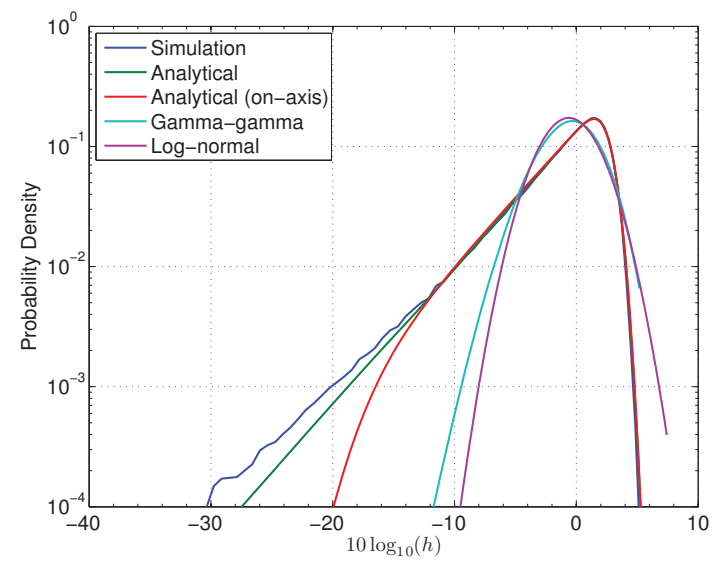

(c) $L=2.5 \mathrm{Km}, C_{n}^{2}=5 \times 10^{-15} \mathrm{~m}^{-2 / 3}$.

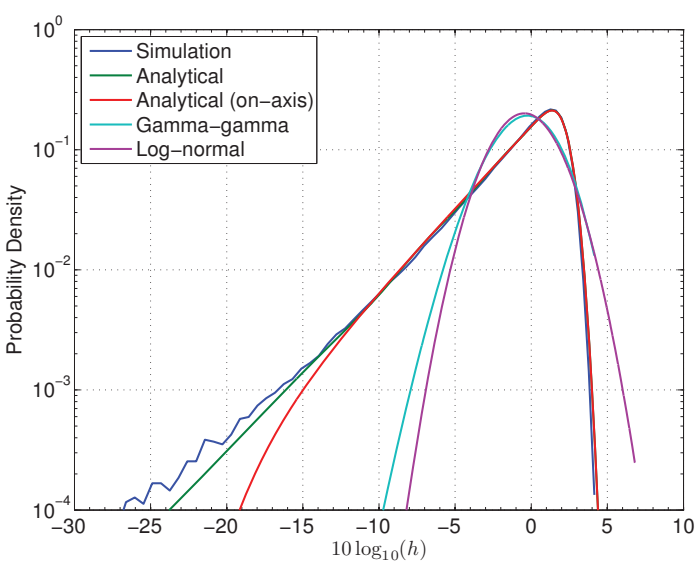

(b) $L=2 \mathrm{Km}, C_{n}^{2}=5 \times 10^{-15} \mathrm{~m}^{-2 / 3}$.

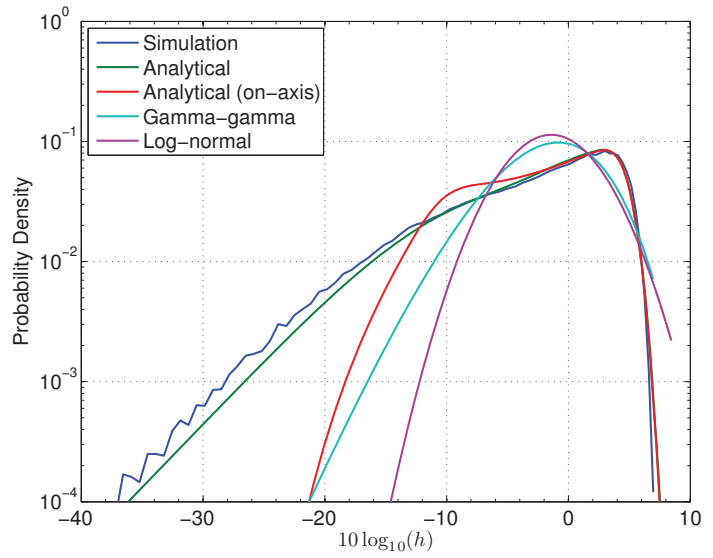

(d) $L=0.75 \mathrm{Km}, C_{n}^{2}=5 \times 10^{-14} \mathrm{~m}^{-2 / 3}$.

Figure 3.5: Overall fading pdfs comparison for focused beam.

variance with the simulated overall fading pdf [38]. It shows that both LN fading pdf and best fitted GG fading pdf largely deviate from the simulated fading pdf in all the studied turbulence channels with focused laser beam. The deep fading tail is especially underestimated by both LN and GG fading pdf models. For $C_{n}^{2}=5 \times 10^{-15} \mathrm{~m}^{-2 / 3}$, at $L=1 \mathrm{Km}$, both the direct numerical wave-optics simulated fading pdf and the analytically calculated fading pdf estimate the probability density of the FSO communication system to experience $-13 \mathrm{~dB}$ fading is about $10^{-4}$. The probability density of the $-13 \mathrm{~dB}$ fading estimated by the best fitted GG model and the LN model is negligible. Instead, the best fitted GG model estimates the deep fading probability density reduces to $10^{-4}$ as early as the fading 


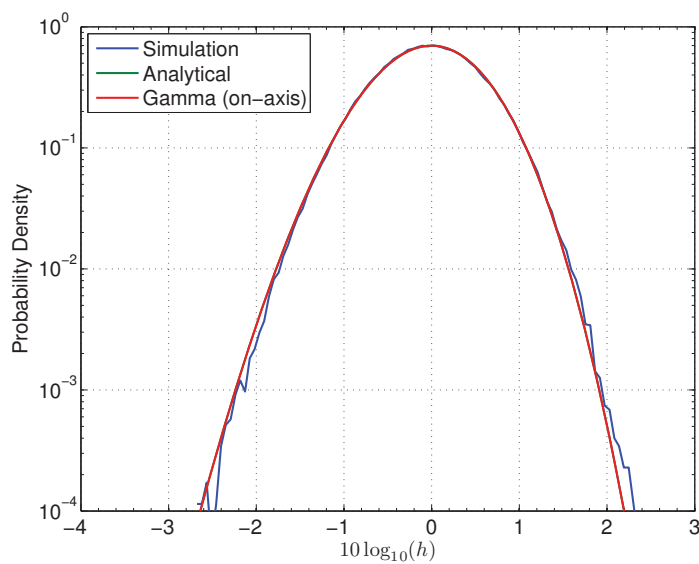

(a) $L=500 \mathrm{~m}, C_{n}^{2}=5 \times 10^{-15} \mathrm{~m}^{-2 / 3}$.

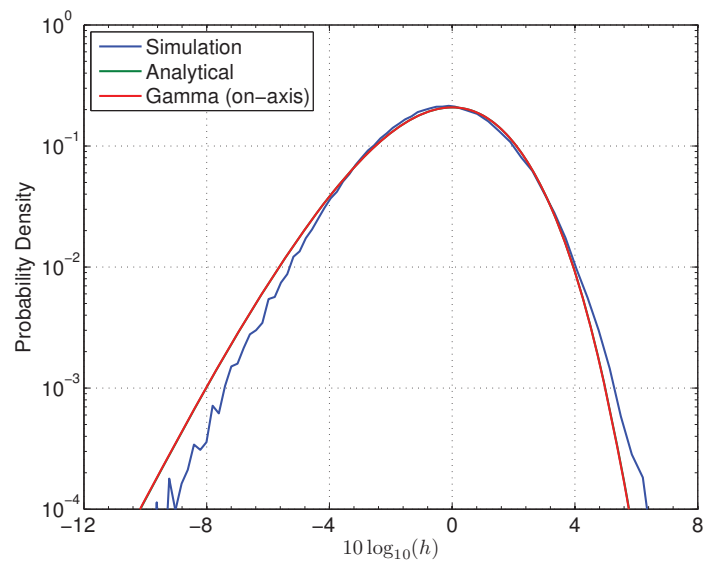

(c) $L=2 \mathrm{Km}, C_{n}^{2}=5 \times 10^{-15} \mathrm{~m}^{-2 / 3}$.

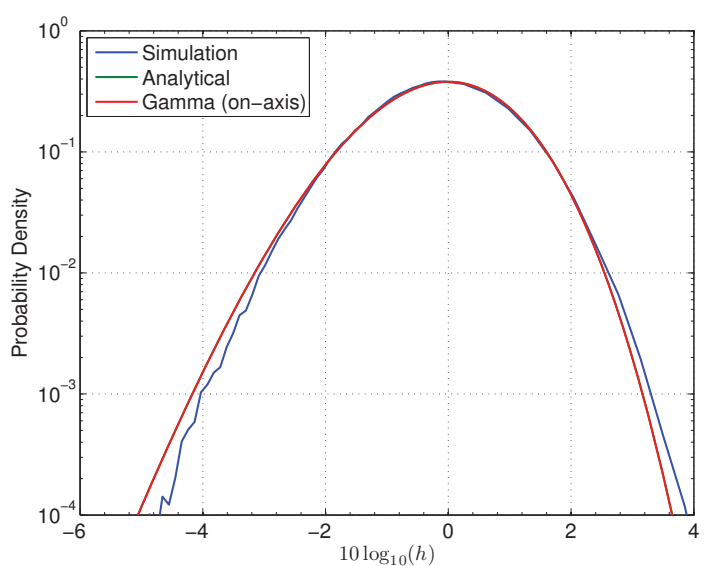

(b) $L=1 \mathrm{Km}, C_{n}^{2}=5 \times 10^{-15} \mathrm{~m}^{-2 / 3}$.

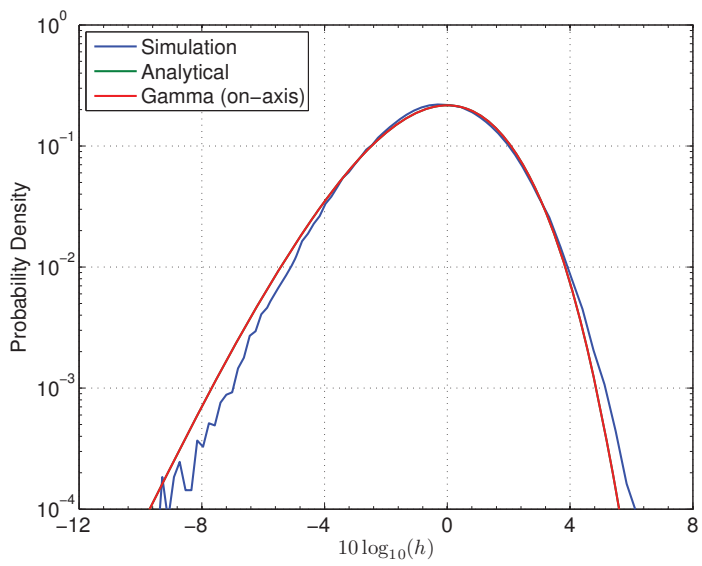

(d) $L=500 \mathrm{~m}, C_{n}^{2}=5 \times 10^{-14} \mathrm{~m}^{-2 / 3}$.

Figure 3.6: Overall fading pdfs comparison for diverged beam.

is only $-6 \mathrm{~dB}$. And LN estimates the deep fading probability density reduces to $10^{-4}$ as early as the fading is only $-5 \mathrm{~dB}$. In the mean while, both the simulated fading pdf and the calculated fading pdf estimate the probability density the system experience $-5 \mathrm{~dB}$ fading is around $10^{-2}$. The probability density estimated by the LN model is 100 times lower. The probability density estimated by best fitted GG model is around $6 \times 10^{-4}$. It is better than that estimated by the LN model but is still 16.7 times lower than that estimated by the simulated and analytically calculated fading pdfs. As the propagation distance increases, the probability density of the system experiences deep fading estimated by all the models increases. However, the probability density of the system experienced deep fading is 
still underestimated by both GG model and LN model. When the propagation distance $L$ increases from $1 \mathrm{Km}$ to $2.5 \mathrm{Km}$, both simulated fading pdf and the analytically calculated fading pdf estimate that the probability density of the system to experience $-28 \mathrm{~dB}$ deep power fading is around $10^{-4}$. The best fitted GG model estimates the deep fading probability density reduces to $10^{-4}$ when the fading is $-12 \mathrm{~dB}$. And LN estimates the deep fading probability density reduces to $10^{-4}$ when the fading is only $-10 \mathrm{~dB}$. At $-10 \mathrm{~dB}$ fading, the probability density estimated by both simulated fading pdf and the analytically calculated fading pdf is around $10^{-2}$. The probability density estimated by the $\mathrm{LN}$ model at $-10 \mathrm{~dB}$ is about 100 times lower. The probability density estimated by the GG model at -10dB fading is about $5 \times 10^{-4}$, which is about 20 times lower than that from the simulated and analytical calculated fading pdfs. The similar underestimation of the deep fading probability density is also observed in the $C_{n}^{2}=5 \times 10^{-14} \mathrm{~m}^{-2 / 3}, L=0.75 \mathrm{Km}$ channel. It should also be noted that the right part of the fading pdf estimated by the best fitted GG model and LN model are higher than that estimated by the simulated and analytically calculated fading pdfs. Hence both the best fitted GG model and LN model not only underestimate the deep fading probability density, they also overestimate the probability density of the system to receiver high beam power. As the deep fading part of the fading pdf is essential to determine the system BER performance, the system BER performance will be largely underestimated by GG and LN models.

For FSO communication system with diverged laser beam, as shown in figure 3.4(a), 3.4(b), 3.4(c), and 3.4(d), the variance of the fast-tracked fading changes little in most of the beam wander ranges (three times $\left.\sigma_{w}\right)$. In $2.10, P_{t}\left(\frac{I_{\text {overall }}}{I_{\text {displace }}} \mid I_{\text {displace }}\right)$ can be approximated by the fast-tracked on-axis fading pdf $P_{t_{-o}}\left(\frac{I_{\text {overall }}}{I_{\text {displace }}}\right)$,

$$
P_{\text {overall }}\left(I_{\text {overall }}\right)=\int_{0}^{\infty} P_{t_{-} o}\left(\frac{I_{\text {overall }}}{I_{\text {displace }}}\right) \frac{P_{d}\left(I_{\text {displace }}\right)}{I_{\text {displace }}} d I_{\text {displace }}
$$

Further, in all the studied diverged beam cases, $I_{\text {displace }}$ only changes a little in most of the beam wander ranges. For each $I_{\text {overall }}, P_{t_{-} o}\left(\frac{I_{\text {overall }}}{I_{\text {displace }}}\right)$ can be approximated to be unchanged 
in the integration region before $\frac{P_{d}\left(I_{\text {displace }}\right)}{I_{\text {displace }}}$ reduces to around zero due to the fast drop of $P_{d}\left(I_{\text {displace }}\right)$ outside the Gaussian beam wander region. Hence

$$
P_{\text {overall }}\left(I_{\text {overall }}\right)=P_{t}\left(\frac{I_{\text {overall }}}{I_{\text {displace }}}\right) \int_{0}^{\infty} \frac{P_{d}\left(I_{\text {displace }}\right)}{I_{\text {displace }}} d I_{\text {displace }}
$$

and as

$$
\int_{0}^{\infty} \frac{P_{d}\left(I_{\text {displace }}\right)}{I_{\text {displace }}} d I_{\text {displace }}=1 / I_{\text {mean }}
$$

where $I_{\text {mean }}$ is the mean value of the overall power fading. Hence

$$
P_{\text {overall }}\left(I_{\text {overall }}\right)=P_{t}\left(\frac{I_{\text {overall }}}{I_{\text {displace }}}\right) / I_{\text {mean }}
$$

and $1 / I_{\text {mean }}$ is eliminated when the overall fading pdf is normalized to it mean value.

Figure 3.6(a), 3.6(b), 3.6(c), and 3.6(d) show the calculated overall fading pdfs of normalized received beam power with point-like receiver for Gaussian diverged beam with $L / F=-1$ at $L=0.5 \mathrm{Km}, L=1 \mathrm{Km}$ and $L=2 \mathrm{Km}$ with $C_{n}^{2}=5 \times 10^{-15} \mathrm{~m}^{-2 / 3}$ and $L=$ $0.5 \mathrm{Km}$ with $C_{n}^{2}=5 \times 10^{-14} \mathrm{~m}^{-2 / 3}$. The calculated overall fading pdfs are compared with the direct wave-optics numerically simulated fading pdfs and gamma approximation of the fast-tracked on-axis fading pdf. As shown in 3.4, the calculated overall fading pdfs are almost identical with the fast-tracked on-axis gamma pdfs when the beam is well diverged. Both the fast-tracked on-axis gamma pdfs and the calculated overall fading pdfs fit well with the directly simulated overall fading pdfs in all the studied propagation distances and turbulence strengths with diverged beam. 


\subsection{Conclusion}

For Gaussian laser beam transmitted through the atmospheric turbulence channel with ether a focused or diverged beam angle, the overall fading pdf of the received beam power by point-like receiver with beam wander is calculated by the analytical method. The analytically calculated fading pdfs accurately fit the fading pdfs obtained from direct large-scale numerical wave-optics simulations under different turbulence strengths, propagation distances, and beam diverging angles. According to the analytical method, the overall fading pdf is calculated based on the fast-tracked on-axis and off-axis fading pdfs, the fast-tracked beam profile, and the pointing error. Large-scale wave-optics numerical simulations show the fast-tracked on-axis and off-axis fading pdfs obtained with point-like receiver can also be modeled by the gamma pdf model. When the laser beam is focused, the fast-tracked fading pdf changes rapidly as the receiver aperture moves apart from the fast-tracked beam center. Both on-axis and off-axis fast-tracked fading pdfs in the beam wander range contribute to the overall fading pdf. When the beam diverging angle increases, the fast-tracked fading pdf becomes to change slower. The size of the fast-tracked beam profile also increases as the transmitted laser beam diverging angle increase. In both focused and diverged beam cases, the radius of the fast-tracked beam profile is close to the radius of the corresponding diffraction pattern. The radius of the fast-tracked beam profile is a little larger due to the turbulence induced beam broadening effect. In this chapter, the pointing error is the turbulence induced beam wander. While the fast-tracked fading pdf changes slower and the fast-tracked beam profile grows larger, the turbulence induced beam wander slightly decreases as the beam diverging angle increases. When the beam spot size is large enough, both the on-axis and off-axis fast-tracked normalized fading pdfs in the beam wander range are almost identical. The overall fading pdf can be well approximated by the fast-tracked on-axis fading pdf. In this case, for the point-like receiver aperture, a single gamma pdf model is adequate to represent the overall fading pdf with the variance of the normalized random power fading of the received laser beam. It is also needed to mention 
that although this chapter studies the FSO communication system with point-like receiver aperture and with pointing error as the turbulence induced beam wander, the results can be expanded to the case with larger receiver aperture and with more general pointing error as indicated in chapter 2. 


\section{Chapter 4}

\section{Fading pdf Models Comparison}

\subsection{Introduction}

In this chapter, the previously proposed heuristic fading pdf models and the analytical method are systematically compared with directly simulated fading pdfs provided by high precision large-scale wave-optics numerical simulations under different turbulence strengths, propagation distances, receiver aperture sizes and pointing errors. The mathematical forms of the previously proposed heuristic fading pdf models are mentioned in chapter 1 . The simulated fading pdfs with largely improved simulation precision provide more details and accuracy than the fading pdfs obtained in the previously published papers when the previously proposed heuristic fading pdf models are proposed and compared. The advantages, impediments and working conditions of each mathematical model are carefully compared and concluded. In the simulation, the FSO system uses the collimated Gaussian laser beam along a horizontal direction. The laser wavelength is $\lambda=1.54 \times 10^{-6} \mathrm{~m}$. The simulations are performed under different turbulence strengths, propagation distances, receiver aperture sizes, and pointing errors. The refractive index structure parameter $C_{n}^{2}$ ranges from 
$5 \times 10^{-15} \mathrm{~m}^{-2 / 3}$ to $1 \times 10^{-13} \mathrm{~m}^{-2 / 3}$. The propagation distance $L$ ranges from $0.4 \mathrm{Km}(0.25$ mile) to $4 \mathrm{Km}$ ( 2.5 miles). The receiver aperture size ranges from point-like $0 \mathrm{~cm}$ aperture to aperture with $20 \mathrm{~cm}$ diameter. The Rytov variance ranges from 0.24 to 1.27 . The simulations cover from weak to strong fluctuation regime. To accurately obtain especially the deep fading tail of the fading pdf, the simulations are conducted under large grid size with high resolution. The field grid size $N \times N$ ranges from $1024 \times 1024$ to $2048 \times 2048$. The field resolution $d x$ ranges from $0.625 \mathrm{~mm}$ to $2.5 \mathrm{~mm}$, which is much smaller than the corresponding coherence radius in each case. The phase screen number $N_{p h z}$ ranges from 10 to 12 . The iteration number $M$ ranges from 0.3 million to 1 million. Two types of the pointing error are studied. They are namely the turbulence induced beam wander only pointing error and the more general pointing error. The comparison shows that only the analytical method accurately fits the directly simulated overall fading pdf of the normalized received beam power of the FSO communication system under different turbulence strengths, propagation distances, receiver aperture sizes and pointing errors.

The rest of this chapter is organized as follows. Section 4.2 compares the fading pdf models with the direct simulated fading pdf under difference turbulence strengths, propagation distances, receiver aperture sizes, and pointing errors. The average bit error rate (BER) and outage probability performance estimated by the fading pdf models and the simulations are compared in section 4.3. Discussions are given in section 4.4 and conclusions are given in section 4.5 .

\subsection{Comparison with Simulation Data}

The mathematical fading pdf models are compared with directly simulated fading pdfs. The simulations provide higher precision than the these of earlier published fading data. With the higher precision, the fading pdf models, especially the deep fading tails, can be 
more accurately verified. The mathematical fading pdf models are compared and verified in different turbulence strengths, propagation distances, receiver aperture sizes, and pointing error cases for collimated Gaussian beam. In the comparison, as $\mathrm{K}$ fading pdf model only works in strong turbulence regime, and IK fading pdf model reduces to $\mathrm{K}$ fading pdf model in strong turbulence regime, only IK fading pdf model is studied. Further, as LNME fading pdf model only works in strong turbulence regime, and Beckmann fading pdf model reduces to LNME fading pdf model in strong turbulence regime, only Beckmann fading pdf model is compared here.

In this chapter, the collimated Gaussian laser beam is assumed to propagate along a horizontal direction. The Gaussian laser beam radius is $5 \mathrm{~cm}$ at the transmitter plane. The laser wavelength is $\lambda=1.54 \times 10^{-6} \mathrm{~m}$. The aerosol scattering effects caused by haze, rain, snow, and fog are not considered. And the atmospheric turbulence is simulated by 10 to 12 phase screens uniformly placed between the transmitter and the receiver apertures. The Kolmogorov spectrum is used to generate the phase screens. The grid size of the phase screen $N \times N$ ranges from $1024 \times 1024$ to $2048 \times 2048$. The resolution of the phase screen $d x$ ranges from $0.625 \mathrm{~mm}$ to $2.5 \mathrm{~mm}$. The resolution is much smaller than the corresponding coherence radius and satisfies the restrictions to accurately simulate the wave propagation [56]. The iteration number $M$ to directly simulate the fading pdf ranges from 0.3 million to 1 million. The iteration numbers are much larger than those in the previous papers. The sufficient simulation iterations provide enough information to accurately study the fading pdf, especially the deep fading tail.

The diameter of receiver aperture ranges from point-like $0 \mathrm{~cm}$ to $20 \mathrm{~cm}$. The point-like $0 \mathrm{~cm}$ receiver aperture is the smallest receiver aperture that can be achieved in the simulation. It contains only a single pixel. It is much smaller than the corresponding coherence radius and it can be regarded as a point in the optical field. The $20 \mathrm{~cm}$ receiver aperture is much larger than the corresponding coherence radius in all the studied cases. Hence the receiver aperture size ranges from much smaller than the coherence radius to much larger than 
coherence radius.

Two types of pointing errors are considered. They are the beam wander only pointing error and the general pointing error. In the beam wander only pointing error case, the pointing error only consists of the turbulence induced beam wander. The other sources of pointing error and the effect of the fast-tracking system are not considered. This is the pointing error case used in most of previously published papers. However, pointing error can also be caused by the sources such as optical misalignment, mechanical vibrations, and relative movements of the transmitter and the receiver. In another hand, the pointing error can be reduced by the fast-tracking system. With fast-tracking, the turbulence induced beam wander is supposed to be reduced and the extra mechanical pointing error is supposed to be eliminated. Parts of the pointing error remains due to the delay and error in the tracking system as the fast-tracking residual. Hence, in the general pointing error case, the pointing error is more complicate than the turbulence induced beam wander only pointing error. The mathematical fading pdf models are also compared and verified in the general pointing error cases.

\subsubsection{Beam Wander Only Pointing Error}

In the beam wander only pointing error case, the propagation distance $L$ ranges from $1.6 \mathrm{Km}$ ( 1 mile) to $4 \mathrm{Km}\left(2.5\right.$ miles). $C_{n}^{2}$ is $5 \times 10^{-15} \mathrm{~m}^{-2 / 3}$. The Rytov variance $\sigma_{R}^{2}$ ranges from 0.24 to 1.27 . The simulations cover both weak and strong turbulence regimes. The corresponding Rytov variance $\sigma_{R}^{2}$ and the coherence radius $r_{0}$ are shown in table 4.1. The simulation parameters, including simulation resolution $d x$, grid size $N$, phase screen number $N_{p h z}$ and iteration number $M$ are shown in table 4.2.

Figure 4.1 shows the comparison of fading pdf models with the directly simulated fading pdfs when the pointing error is the turbulence induced beam wander. The fading pdf is on 
Table 4.1: Rytov variance and coherence radius.

\begin{tabular}{cccc}
\hline $\begin{array}{c}L \\
(\mathrm{Km})\end{array}$ & $\begin{array}{c}C_{n}^{2} \\
\left(\mathrm{~m}^{-2 / 3}\right)\end{array}$ & $\sigma_{R}^{2}$ & $\begin{array}{c}r_{0} \\
(\mathrm{~cm})\end{array}$ \\
\hline 1.6 & $5 \times 10^{-15}$ & 0.24 & 4.2 \\
\hline 3.2 & $5 \times 10^{-15}$ & 0.85 & 2.8 \\
\hline 4 & $5 \times 10^{-15}$ & 1.27 & 2.4 \\
\hline
\end{tabular}

Table 4.2: Simulation parameters for beam wander only pointing error case.

\begin{tabular}{ccccc}
\hline $\begin{array}{c}L \\
(\mathrm{Km})\end{array}$ & $\begin{array}{c}d x \\
(\mathrm{~mm})\end{array}$ & $N$ & $N_{p h z}$ & $\begin{array}{c}M \\
\left(10^{6}\right)\end{array}$ \\
\hline 1.6 & 2.5 & 1024 & 10 & 0.308 \\
\hline 3.2 & 1.75 & 1536 & 12 & 0.722 \\
\hline 4 & 2.5 & 1024 & 12 & 0.67
\end{tabular}

$10 \log _{10}(h)$ scale to emphasize on the deep fading tail, where $h$ is the normalized channel state. With $C_{n}^{2}=5 \times 10^{-15} \mathrm{~m}^{-2 / 3}$, at $L=1.6 \mathrm{Km}$, the Rytov variance $\sigma_{R}^{2}$ is 0.24 . The system is in the weak turbulence regime. In this weak turbulence channel, when the receiver aperture is the point-like 0cm aperture (figure 4.1(a)), the analytical method, Beckmann fading pdf model and the GG fading pdf model have close fit to the directly simulated fading pdf. LN fading pdf model deviates from the simulated fading pdf. And both IK fading pdf model and IK Weak fading pdf model, which is the approximation of the IK fading pdf model under weak turbulence condition, are quite different from the simulated fading pdf. In figure $4.1(\mathrm{~b})$, the aperture diameter grows to $14 \mathrm{~cm}$, which is much larger than the coherence radius. The analytical method still accurately fits the simulated fading pdf. Both GG and LN fading pdf models deviate from the simulated fading pdf. IK and IK Weak fading pdf models provide relatively good fit to the deep fading tail but divided from the directly simulated fading pdf when $h$ grows higher. In this case, the Beckmann fading pdf model fails to generate the corresponding parameters. Figure 4.1(c) and 4.1(d) show the comparison of the fading pdf models with $L=3.2 \mathrm{Km}$, receiver aperture diameter $D=0 \mathrm{~cm}$ and $D=14 \mathrm{~cm}$, respectively. The corresponding Rytov variance $\sigma_{R}^{2}$ increases to 0.85 . With point-like $0 \mathrm{~cm}$ aperture, both analytical method and the Beckmann fading 
pdf model fit well with the simulated fading pdf. GG fading pdf model begins to deviate from the simulated fading pdf in the deep fading tail. Other fading pdf models are quite different from the simulated fading pdf. When aperture diameter increases to $14 \mathrm{~cm}$, only the analytical method keeps fitting well with the simulated fading pdf. When propagation distance increases to $4 \mathrm{Km}$, the Rytov variance $\sigma_{R}^{2}$ increases to 1.27 . The system is in the strong turbulence regime. With point-like $0 \mathrm{~cm}$ receiver aperture, both analytical method and the Beckmann fading pdf model fit well with the simulated fading pdf. GG fading pdf model deviates further away from the simulated fading pdf in the deep fading tail. When receiver aperture diameter grows to $20 \mathrm{~cm}$, only the analytical method still fits well with the simulated fading pdf.

The comparison shows that in the beam wander only pointing error case, the analytical method shows good fit to the simulated fading pdf with both point-like receiver aperture and large receiver aperture at both weak and strong turbulence regimes. The Beckmann fading pdf model provides good fit to the simulated fading pdf with point-like receiver aperture at both weak and strong turbulence regimes. When the receiver aperture size increases, the Beckmann fading pdf model faces challenges to obtain valid parameters. With pointlike receiver aperture, The GG fading pdf model provides good fit when the propagation distance is short. The GG fading pdf model begins to deviate from the simulated fading pdf at deep fading tail when the propagation distance $L$ increases. The GG fading pdf model approaches the LN fading pdf model when the receiver aperture size grows large. The IK, IK Weak, and LN fading pdf models are quite different from the simulated fading pdf in all the cases. IK and GG fading pdf model faces calculation difficulties when the receiver aperture size grows large. The IK Weak fading pdf model provides better fit to the deep fading tail than the LN fading pdf model although both models are generally different from the simulated fading pdf. 


\subsubsection{General Pointing Error}

In the general pointing error case, the pointing error is extended from the turbulence induced beam wander only pointing error to a more general pointing error. The effects of other mechanical pointing error sources as well as the effects of the fast-tracking system are considered. The fading pdf models are compared with the directly simulated fading pdfs under different turbulence strengths, propagation distances, receiver aperture sizes, and pointing errors.

In the study, $C_{n}^{2}$ ranges from $5 \times 10^{-15} \mathrm{~m}^{-2 / 3}$ to $1 \times 10^{-13} \mathrm{~m}^{-2 / 3}$. The propagation distance $L$ ranges from $0.4 \mathrm{Km}(0.25$ mile) to $4 \mathrm{Km}$ ( 2.5 miles $)$. The corresponding Rytov variance $\sigma_{R}^{2}$ and the coherence radius $r_{0}$ are shown in table 4.3. In the general pointing error case, the simulations are divided to two separate sets. The first set of simulations provide the channel informations needed by the analytical method. The channel informations include fast-tracked on-axis and off-axis fading pdfs, and fast-tracked beam profile. Based on these channel informations, the overall fading pdf with given pointing error can be directly calculated by the analytical method under different pointing errors for each turbulence channel. The second set of the simulations directly simulate the overall fading pdf in each channel with given pointing error. The parameters for the first and second set of the simulations are shown in table 4.4 and 4.5 , respectively. In table $4.5, \Delta_{x}$ and $\Delta_{y}$ are the misalignments in $x$ and $y$ directions, $\sigma_{b}$ is the standard deviation of the turbulence induced beam wander, $\sigma_{p}$ is the standard deviation of the extra pointing error, $\sigma_{d}$ is the standard deviation of the overall pointing error.

The pointing error models considered in the general pointing error case are the beam wander plus extra Gaussian random pointing error model and the fast-tracking residual pointing error model. In the beam wander plus Gaussian random pointing error model, in addition to the turbulence induced beam wander, the overall pointing error is also comprised of the 
extra two dimensional Gaussian pointing error. In this chapter, the extra pointing error is modeled as an independent two dimensional Gaussian distributed variable. In the fasttracking residual error model, the extra pointing error is eliminated and the turbulence induced beam wander is also reduced by the fast-tracking system. The remained fast-tracking residual error is assumed to follow the two dimensional Gaussian distribution.

Table 4.3: Rytov variance and coherence radius.

\begin{tabular}{cccc}
\hline $\begin{array}{c}L \\
(\mathrm{Km})\end{array}$ & $\begin{array}{c}C_{n}^{2} \\
\left(\mathrm{~m}^{-2 / 3}\right)\end{array}$ & $\sigma_{R}^{2}$ & $\begin{array}{c}r_{0} \\
(\mathrm{~cm})\end{array}$ \\
\hline 0.4 & $1 \times 10^{-13}$ & 0.37 & 1.6 \\
\hline 1.6 & $5 \times 10^{-15}$ & 0.24 & 4.2 \\
\hline 4 & $5 \times 10^{-15}$ & 1.27 & 2.4 \\
\hline
\end{tabular}

Table 4.4: Simulation parameters for channel information in general pointing error case.

\begin{tabular}{cccccc}
\hline $\begin{array}{c}L \\
(\mathrm{Km})\end{array}$ & $\begin{array}{c}C_{n}^{2} \\
\left(\mathrm{~m}^{-2 / 3}\right)\end{array}$ & $N$ & $\begin{array}{c}d x \\
(\mathrm{~mm})\end{array}$ & $\begin{array}{c}M \\
\left(10^{6}\right)\end{array}$ & $N_{p h z}$ \\
\hline 0.4 & $1 \times 10^{-13}$ & 2048 & 0.625 & 0.12 & 10 \\
\hline 1.6 & $5 \times 10^{-15}$ & 1024 & 2.5 & 0.31 & 10 \\
\hline 4 & $5 \times 10^{-15}$ & 1024 & 2.5 & 0.67 & 12 \\
\hline
\end{tabular}

Table 4.5: Direct simulation parameters for overall fading with general pointing error case.

\begin{tabular}{cccccccccccc}
\hline $\begin{array}{c}L \\
(\mathrm{Km})\end{array}$ & $\begin{array}{c}\text { pointing error } \\
\text { model }\end{array}$ & $\begin{array}{c}C_{n}^{2} \\
\left(\mathrm{~m}^{-2 / 3}\right)\end{array}$ & $\begin{array}{c}\Delta x \\
(\mathrm{~cm})\end{array}$ & $\begin{array}{c}\Delta y \\
(\mathrm{~cm})\end{array}$ & $\begin{array}{c}\sigma_{b} \\
(\mathrm{~cm})\end{array}$ & $\begin{array}{c}\sigma_{p} \\
(\mathrm{~cm})\end{array}$ & $\begin{array}{c}\sigma_{d} \\
(\mathrm{~cm})\end{array}$ & $N$ & $\begin{array}{c}d x \\
(\mathrm{~mm})\end{array}$ & $\begin{array}{c}M \\
\left(10^{6}\right)\end{array}$ & $N_{p h z}$ \\
\hline 0.4 & Gaussian & $1 \times 10^{-13}$ & 0 & 0 & 0.32 & 1.25 & 1.29 & 2048 & 0.625 & 0.41 & 10 \\
\hline 1.6 & Gaussian & $5 \times 10^{-15}$ & 0 & 0 & 0.58 & 2.50 & 2.57 & 1024 & 2.5 & 0.56 & 10 \\
\hline 1.6 & Gaussian & $5 \times 10^{-15}$ & 1.25 & 1.25 & 0.58 & 2.50 & 2.57 & 1024 & 2.5 & 1 & 10 \\
\hline 1.6 & Gaussian & $5 \times 10^{-15}$ & 2.5 & 2.5 & 0.58 & 2.50 & 2.57 & 1024 & 2.5 & 0.70 & 10 \\
\hline 4 & Residual & $5 \times 10^{-15}$ & 0 & 0 & - & - & 2.00 & 1536 & 1.6 & 0.49 & 12 \\
\hline 4 & Gaussian & $5 \times 10^{-15}$ & 0 & 0 & 2.29 & 1.00 & 2.50 & 1024 & 2.5 & 1 & 12 \\
\hline
\end{tabular}

In the general pointing error case, three propagation distances are studied. They are $L=$ $0.4 \mathrm{Km}, L=1.6 \mathrm{Km}$ and $L=4 \mathrm{Km}$. For propagation distance $L=0.4 \mathrm{Km}$, the overall fading pdf is studied with high $C_{n}^{2}$ where $C_{n}^{2}=1 \times 10^{-13} \mathrm{~m}^{-2 / 3}$. The pointing error is the beam 
wander plus extra Gaussian pointing error. The extra pointing error is assumed to follow the two dimensional Gaussian distribution with zero mean and variance $\sigma_{p}=1.25 \mathrm{~cm}$ in both $x$ and $y$ directions. As the turbulence induced beam wander also follows the two dimensional Gaussian distribution with zero mean and standard derivation $\sigma_{b}$ [21], [23], the overall pointing error follows the two dimensional Gaussian distribution with zero mean and standard derivation $\sigma_{d}$, where $\sigma_{d}=\sqrt{\sigma_{p}^{2}+\sigma_{b}^{2}}=1.29 \mathrm{~cm}$. As shown in figure 4.2(a), with the point-like $0 \mathrm{~cm}$ receiver aperture, the analytical method accurately fits the simulated fading pdf. With this relatively small extra pointing error, the Beckmann fading pdf model maintains relatively good fit under this point-like receiver aperture. The GG fading pdf model underestimates the deep fading probability. LN fading pdf model predicts an even lower deep fading probability. The IK and IK Weak fading pdf models are different from the simulated fading pdf. When the receiver aperture diameter $D$ increases to $10 \mathrm{~cm}$, only the analytical method accurately fits well with the directly simulated fading pdf. IK and IK Weak fading pdf models provide relatively good fit to the deep fading tail but are different from the directly simulated fading pdf when $h$ grows higher. Both GG and LN fading pdf model are quite different from the directly simulated fading pdf. The Beckmann fading pdf model fails to generate the valid parameter.

For propagation distance $L=1.6 \mathrm{Km}, C_{n}^{2}=5 \times 10^{-15} \mathrm{~m}^{-2 / 3}$. The pointing error is the turbulence induced beam wander plus extra pointing error. Three extra Gaussian pointing errors are studied. They are extra Gaussian pointing error with zero mean and standard deviation $\sigma_{p}=2.5 \mathrm{~cm}$ in both $x$ and $y$ directions (figure 4.3(a) and 4.3(b)); extra Gaussian pointing error with $1.25 \mathrm{~cm}$ mean and standard deviation $\sigma_{p}=2.5 \mathrm{~cm}$ in both $x$ and $y$ directions (figure 4.3(c) and 4.3(d)); extra Gaussian pointing error with larger $2.5 \mathrm{~cm}$ mean and standard deviation $\sigma_{p}=2.5 \mathrm{~cm}$ in both $x$ and $y$ directions (figure 4.3(e) and 4.3(f)). It is shown that only the analytical method accurately fits the simulated fading pdf under different misalignment conditions and receiver aperture sizes. All other fading pdf models show different shape comparing to the directly simulated fading pdfs under different receiver aperture sizes in all the cases. 
Figure 4.4 and 4.5 show the comparison of the fading pdf models with the simulated fading pdfs for $L=4 \mathrm{Km}, C_{n}^{2}=5 \times 10^{-15} \mathrm{~m}^{-2 / 3}$ with fast-tracking residual error and beam wander plus extra Gaussian pointing error, respectively. The Rytov variance $\sigma_{R}^{2}=1.27$. It is in the strong turbulence regime. In the fast-tracking residual error case, the extra pointing error is assumed to be well eliminated and the turbulence induced beam wander is assumed to be reduced by the fast-tracking system. The fast-tracking residual error is assumed to follow the two dimensional Gaussian distribution with zero mean and standard deviation $\sigma_{d}=2 \mathrm{~cm}$ in both $x$ and $y$ directions. In the beam wander plus extra Gaussian pointing error case, the extra pointing error is assumed to follow the two dimensional Gaussian distribution with zero mean and standard deviation $\sigma_{p}=1 \mathrm{~cm}$ in both $x$ and $y$ directions. The overall pointing error follows the two dimensional Gaussian distribution with zero mean and standard deviation $\sigma_{d}=2.5 \mathrm{~cm}$ in both $x$ and $y$ directions. It is shown that the analytical method fits well with directly simulated fading pdf in both pointing error cases and all the receiver aperture sizes under this strong turbulence. With point-like $0 \mathrm{~cm}$ receiver aperture, both Beckmann and GG fading pdf models deviates from the simulated fading pdf in both reduced and increased pointing error cases. IK, IK Weak and LN fading pdf models are different from the simulated fading pdf. With $20 \mathrm{~cm}$ receiver aperture, Beckmann fading pdf model fails to generate valid parameters in both pointing error cases. IK fading pdf model fails to generate valid parameters in figure 4.5(b).

\subsection{Average BER and Outage Probability}

After the careful comparison between the fading pdf models and the simulated fading pdf, the next question is how the difference will affect the system performance estimation. The system average BER performance and outage probability estimated by different fading pdf models are compared. It is needed to mention that as the deep fading probability density estimated IK fading pdf model increases as fading goes deeper in some cases, the IK fading 
pdf model is not suitable to estimate the system performance.

Assume the system model is

$$
y=h x+n
$$

where $y$ is the received instantaneous signal, $h$ is the normalized channel fading, $x$ is the average received signal, $n$ is the equivalent Gaussian noise, and $n \sim N\left(0, \sigma_{n}^{2}\right)$. Assume the signal is modulated by OOK modulation where

$$
x=\left\{\begin{array}{cc}
\bar{x}, & \text { send } 1 \\
0, & \text { send } 0
\end{array}\right.
$$

where $\bar{x}$ is the average received power when ' 1 ' is transmitted. Assume the probabilities to send signal ' 0 ' and signal ' 1 ' are equal, hence $P(x=0)=P(x=\bar{x})=1 / 2$. Assume the receiver has the knowledge of the instant channel state $h$, given Gaussian noise $n$, the optimal threshold $T_{h}$ that minimize the error probability is $T_{h}=h \bar{x} / 2$. Denote

$$
A=\frac{1}{2} \bar{x},
$$

the instantaneous error probability $P_{e}(h)$ after optimal thresholding is [54]

$$
P_{e}(h)=Q\left(\frac{h \bar{x}}{2 \sigma_{n}}\right)=Q\left(\frac{h A}{\sigma_{n}}\right)
$$

Hence

$$
\bar{P}_{e}=\int P_{e}(h) P(h) d h
$$

where $\bar{P}_{e}$ is the average BER. 
Assuming $\sigma_{n}^{2}=1$, the average BER performance is compared at propagation distance $L=$ $1.6 \mathrm{Km}$ and $L=4 \mathrm{Km}$ with $C_{n}^{2}=5 \times 10^{-15} \mathrm{~m}^{-2 / 3}$ under both turbulence induced beam wander only pointing error and the general pointing error. Figure 4.6(a) and figure 4.6(b) show the average BER obtained in the $1.6 \mathrm{Km}$ channel with Gaussian extra pointing error with $\Delta x=\Delta y=0 \mathrm{~cm}$ and the standard deviation of the overall pointing error as $\sigma_{d}=2.57 \mathrm{~cm}$. Figure 4.6(c) and figure 4.6(d) show the average BER obtained in the $1.6 \mathrm{Km}$ channel with Gaussian extra pointing error with $\Delta x=\Delta y=2.5 \mathrm{~cm}$ and the standard deviation of the overall pointing error as $\sigma_{d}=2.57 \mathrm{~cm}$. The corresponding fading pdfs are shown in figure 4.3(a), 4.3(b), 4.3(e), and 4.3(f), respectively.

Figure 4.7(a) and figure 4.7(b) show the average BER obtained in the $4 \mathrm{Km}$ channel with turbulence induced beam wander only pointing error with $\Delta x=\Delta y=0 \mathrm{~cm}$ and $\sigma_{b}=2.29 \mathrm{~cm}$. Figure 4.7(c) and figure 4.7(d) show the average BER obtained in the $4 \mathrm{Km}$ channel with Gaussian extra pointing error with $\Delta x=\Delta y=0 \mathrm{~cm}$ and the standard deviation of the overall pointing error as $\sigma_{d}=2.5 \mathrm{~cm}$. The corresponding fading pdfs are shown in figure 4.1(e), 4.1(f) and figure 4.5(a), 4.5(b), respectively.

As shown in figure 4.6, the average BER increases as the misalignment (average pointing error) increases from $0 \mathrm{~cm}$ to $2.5 \mathrm{~cm}$ in both $x$ and $y$ directions. Only the analytical method accurately estimates the average BER under all the pointing error cases and receiver aperture sizes. Both the GG fading pdf model and LN fading pdf model underestimate the BER in all the receiver aperture sizes and misalignment cases. The Beckmann fading pdf model is not available due to it fails to generate valid parameters. When the valid parameters are generated in figure 4.6(c), the Beckmann has better BER estimation than both GG and LN fading pdf models. But it still underestimates the BER performance in this general pointing error case. The average BER curve estimated by the IK Weak fading pdf model is also different from the simulated fading pdf. In figure 4.7, the average BER increases as the pointing error variance increases from the turbulence induced beam wander only pointing error case to the beam wander plus extra Gaussian pointing error case. The ana- 
lytical method accurately estimates the average BER under all the pointing error cases and receiver aperture sizes. When the pointing error is the turbulence induced beam wander only pointing error, with point-like $0 \mathrm{~cm}$ receiver aperture, the Beckmann fading pdf model accurately estimates the average BER performance. And the Beckmann fading pdf model fails in other situations with more general pointing errors or with larger receiver apertures. The GG fading pdf generally underestimates the average BER. The LN fading pdf model predicts even lower BER. For point-like 0cm receiver aperture, IK Weak fading pdf model generally overestimates the average BER. For $20 \mathrm{~cm}$ receiver aperture, IK Weak fading pdf model has slight closer estimation than the GG and LN fading pdf models. This is due to the better fit of the deep fading tails by the IK Weak fading pdf model under this large aperture. The average BER curves estimated by GG and LN fading pdf models are almost identically in both pointing error cases with this large receiver aperture.

Table 4.6: Probability (\%) for $10 \mathrm{~dB}$ outage. $L=1.6 \mathrm{Km} . C_{n}^{2}=5 \times 10^{-15} \mathrm{~m}^{-2 / 3}$. Gaussian extra pointing error model. $\Delta x=\Delta y=0 \mathrm{~cm}$, and $\sigma_{b}=0.58 \mathrm{~cm}, \sigma_{p}=$ $2.5 \mathrm{~cm}, \sigma_{d}=2.57 \mathrm{~cm}$ in both $x$ and $y$ directions.

\begin{tabular}{cccccc}
\hline \multirow{2}{*}{ Model } & \multicolumn{5}{c}{ Aperture $D(\mathrm{~cm})$} \\
\cline { 2 - 6 } & 0 & 5 & 7 & 10 & 14 \\
\hline Simulation & 5.96 & 2.64 & 1.43 & 0.41 & 0.04 \\
\hline Analytical & 5.90 & 2.62 & 1.43 & 0.41 & 0.04 \\
\hline GG & 1.68 & 1.14 & 0.01 & 0.00 & 0.00 \\
\hline LN & 0.05 & 0.00 & 0.00 & 0.00 & 0.00 \\
\hline IK Weak & 5.30 & 2.32 & 1.18 & 0.21 & 0.00 \\
\hline
\end{tabular}

Table 4.6 and table 4.7 show the probabilities of the $10 \mathrm{~dB}$ outage for the $1.6 \mathrm{Km}$ channel with pointing error as beam wander plus extra Gaussian pointing error with no misalignment and with $2.5 \mathrm{~cm}$ misalignment in both $x$ and $y$ directions, respectively. Table 4.8 and table 4.9 show the probabilities of the $10 \mathrm{~dB}$ outage for the $4 \mathrm{Km}$ channel with pointing error as beam wander only pointing error and beam wander plus extra Gaussian pointing error, respectively. It is shown that the analytical method accurately estimates the outage probability in all the aperture sizes and pointing errors. When the pointing error is the 
Table 4.7: Probability (\%) for 10dB outage. $L=1.6 \mathrm{Km} . C_{n}^{2}=5 \times 10^{-15} \mathrm{~m}^{-2 / 3}$. Gaussian extra pointing error model. $\Delta x=\Delta y=2.5 \mathrm{~cm}$, and $\sigma_{b}=0.58 \mathrm{~cm}, \sigma_{p}=$ $2.5 \mathrm{~cm}, \sigma_{d}=2.57 \mathrm{~cm}$ in both $x$ and $y$ directions.

\begin{tabular}{cccccc}
\hline \multirow{2}{*}{ Model } & \multicolumn{5}{c}{ Aperture $D(\mathrm{~cm})$} \\
\cline { 2 - 6 } & 0 & 5 & 7 & 10 & 14 \\
\hline Simulation & 17.8 & 10.2 & 7.01 & 3.07 & 0.64 \\
\hline Analytical & 17.6 & 10.4 & 7.08 & 3.15 & 0.66 \\
\hline GG & 4.86 & 1.22 & 0.36 & 0.01 & 0.00 \\
\hline Beckmann & 9.89 & - & - & - & - \\
\hline LN & 1.02 & 0.15 & 0.03 & 0.00 & 0.00 \\
\hline IK Weak & 9.44 & 6.59 & 4.82 & 2.17 & 0.25 \\
\hline
\end{tabular}

Table 4.8: Probability (\%) for $10 \mathrm{~dB}$ outage. $L=4 \mathrm{Km} . C_{n}^{2}=5 \times 10^{-15} \mathrm{~m}^{-2 / 3}$. Beam wander only. $\Delta x=\Delta y=0 \mathrm{~cm}$, and $\sigma_{b}=2.29 \mathrm{~cm}, \sigma_{p}=0 \mathrm{~cm}, \sigma_{d}=2.29 \mathrm{~cm}$ in both $x$ and $y$ directions.

\begin{tabular}{cccccc}
\hline \multirow{2}{*}{ Model } & \multicolumn{5}{c}{ Aperture $D(\mathrm{~cm})$} \\
\cline { 2 - 6 } & 0 & 5 & 7 & 10 & 14 \\
\hline Simulation & 4.32 & 0.97 & 0.36 & 0.07 & 0.00 \\
\hline Analytical & 3.66 & 0.90 & 0.34 & 0.06 & 0.00 \\
\hline GG & 3.98 & 0.05 & 0.06 & 0.00 & 0.00 \\
\hline Beckmann & 4.29 & 0.97 & - & - & - \\
\hline LN & 0.22 & 0.01 & 0.00 & 0.00 & 0.00 \\
\hline IK Weak & 6.98 & 3.32 & 1.67 & 0.30 & 0.00 \\
\hline
\end{tabular}

turbulence induced beam wander only pointing error, the Beckmann fading pdf model can predict close outage probabilities when the valid parameters are able to be generated. However, as shown here and also in [35], the valid parameters are not guaranteed. When the pointing error is the general pointing error, the Beckamnn fading pdf model fails to predict an close outage probability even when the parameters are successfully generated (table 4.7, table 4.9). The GG fading pdf model generally underestimates the outage probability. The LN fading pdf model estimates an even lower outage probability. And in the studied cases, the IK Weak fading pdf model shows a largely overestimated outage probability. 
Table 4.9: Probability (\%) for $10 \mathrm{~dB}$ outage. $L=4 \mathrm{Km} . C_{n}^{2}=5 \times 10^{-15} \mathrm{~m}^{-2 / 3}$. Gaussian extra pointing error model. $\Delta x=\Delta y=0 \mathrm{~cm}$, and $\sigma_{b}=2.29 \mathrm{~cm}, \sigma_{p}=1 \mathrm{~cm}$, $\sigma_{d}=2.5 \mathrm{~cm}$ in both $x$ and $y$ directions.

\begin{tabular}{cccccc}
\hline \multirow{2}{*}{ Model } & \multicolumn{5}{c}{ Aperture $D(\mathrm{~cm})$} \\
\cline { 2 - 6 } & 0 & 5 & 7 & 10 & 14 \\
\hline Simulation & 5.14 & 1.45 & 0.69 & 0.16 & 0.01 \\
\hline Analytical & 4.85 & 1.59 & 0.76 & 0.18 & 0.01 \\
\hline GG & 4.69 & 0.60 & 0.07 & 0.00 & 0.00 \\
\hline Beckmann & 2.57 & 1.15 & - & - & - \\
\hline LN & 0.29 & 0.00 & 0.00 & 0.00 & 0.00 \\
\hline IK Weak & 7.43 & 3.77 & 2.03 & 0.46 & 0.01 \\
\hline
\end{tabular}

\subsection{Discussion}

Most of the previous heuristic fading pdf models are doubly stochastic models. K fading pdf model can be regarded as the doubly stochastic model where the conditional fading pdf follows the exponential distribution while the mean value follows the gamma distribution [12]. IK fading pdf model assumes the conditional fading pdf follows the $n$ distribution while the mean value follows the exponential distribution. LNME fading pdf model assumes the conditional fading pdf follows the exponential distribution while the mean value follows the LN distribution. Beckmann fading pdf model assumes the conditional fading pdf follows the Rice-Nakagami distribution and the mean value follows the LN distribution. GG fading pdf model assumes both the modulated stochastic process follow the statistically independent gamma distribution. For LN fading pdf model, although it is governed by a single distribution, it can re regarded as the approximation of the Beckmann fading pdf model in weak turbulence regime [35]. And IK Weak fading pdf model is the approximation of the IK fading pdf model in weak turbulence regime.

In the analytical method, the fast-tracked on-axis and off-axis fading pdfs can be regarded as the conditional distribution. Unlike some fading pdf models use a mathematical distribution with a fixed parameter for the conditional distribution, the analytical method depends 
on both on-axis and off-axis fading pdfs. And the fast-tracked fading pdf changes as the mean value changes with the off-axis location. For the distribution of the random mean value, instead of the adopting a heuristic model, the analytical method points out that the distribution is determined by the pointing error model, the fast-tracked fading profile and the receiver aperture size.

When the pointing error is the turbulence induced beam wander only pointing error, for the point-like receiver aperture, the GG fading pdf model provides relatively good fit to the directly simulated fading pdf when the fading is relatively weak (figure 4.1(a)). This is because the both the stochastic processes can be approximated to gamma distribution in this weak turbulence regime. However, when the propagation distance $L$ grows, The GG fading pdf model begins to underestimate the deep fading probability(figure 4.1(e)). One of the reasons is that when the propagation distance grows, the beam wander effect becomes strong. The fast-tracked off-axis fading pdfs, which generally have larger normalized fading variances, have more contribution to the overall fading pdf and thus increase the deep fading probability. Based on the analytical method, more accurate approximation of the fading pdf model can be derived at different turbulence strength and receiver aperture size regimes.

\subsection{Conclusions}

The analytical method accurately fits the directly simulated fading pdf under different turbulence strengths, propagation distances, receiver aperture sizes and pointing error models. When there is no mechanical induced extra pointing error, and with point-like receiver aperture, Beckmann fading pdf model can well estimate the overall fading pdfs. However, Beckmann fading pdf model generally fails otherwise. Both the analytical method and Beckmann fading pdf model require certain amount of information from the channel. 
For the analytical method, given the fast-tracked on-axis and off-axis fading variance, the fast-tracked beam profile and the pointing error, the overall fading pdfs can be directly calculated. For the Beckmann fading pdf model, the $-1 / 2$ order and the second order moments are required to calculate the parameters of the Beckmann model. However, a solution is not always guaranteed for the Beckmann fading pdf model. With turbulence induced beam wander only pointing error and point-like receiver, the GG fading pdf model provides good fit when the beam wander effect is weak. The GG fading pdf model deviates from the deep fading tail when the beam wander effect becomes strong. The GG fading pdf model are different from the simulated fading pdf under larger receiver aperture or more general pointing error. Although the parameters of the GG fading pdf model are regarded to be connected to the atmospheric turbulence parameters, to better fit the simulated fading pdf, the parameters need to be obtained from the best fit of the simulated fading pdf. The parameters of both the $\mathrm{LN}$ fading pdf model and the approximation of the IK distribution in weak turbulence condition (IK Weak) can be directly obtained from the normalized overall fading variance. However the LN and IK Weak fading pdf model provide the least accurate fit compared with all the fading pdf models mentioned above. When the turbulence induced beam wander is partially compensated or when the mechanical induced extra pointing error is involved, only the analytically calculated fading pdfs provide an accurate match to the direct simulated fading pdfs. 


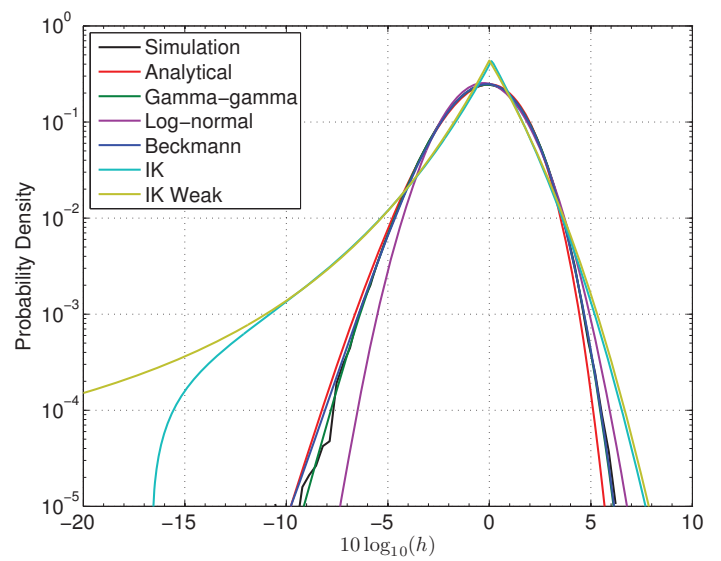

(a) $L=1.6 \mathrm{Km}, D=0 \mathrm{~cm}$

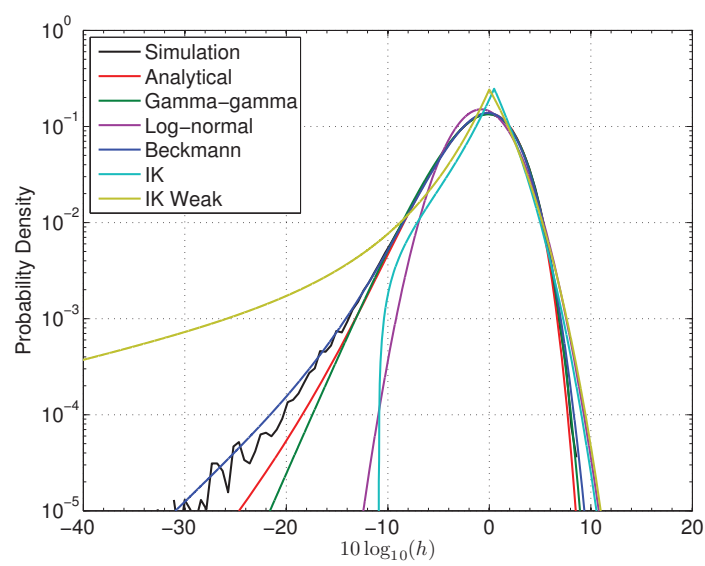

(c) $L=3.2 \mathrm{Km}, D=0 \mathrm{~cm}$

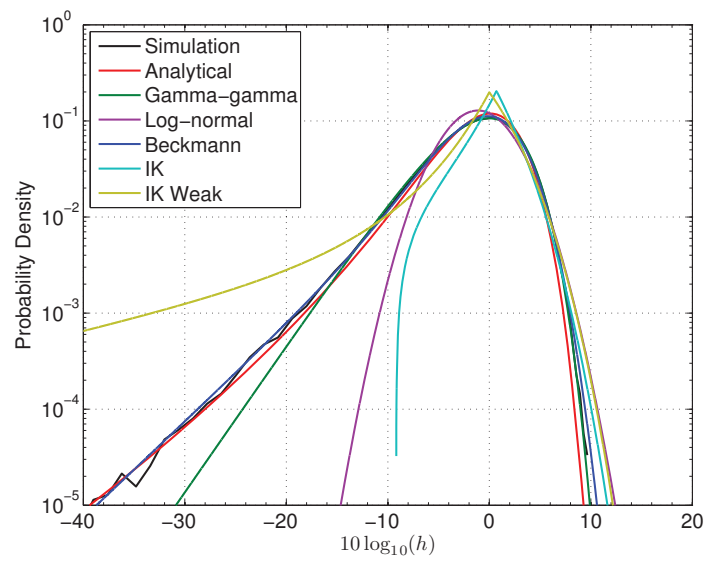

(e) $L=4 \mathrm{Km}, D=0 \mathrm{~cm}$

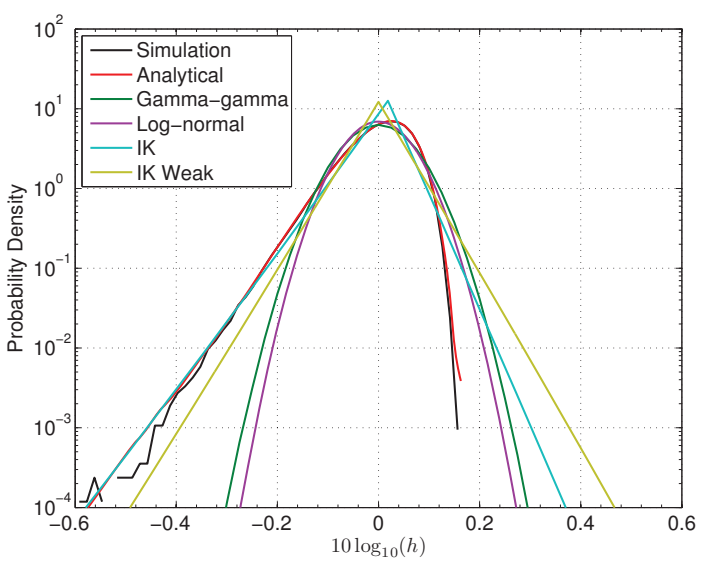

(b) $L=1.6 \mathrm{Km}, D=14 \mathrm{~cm}$

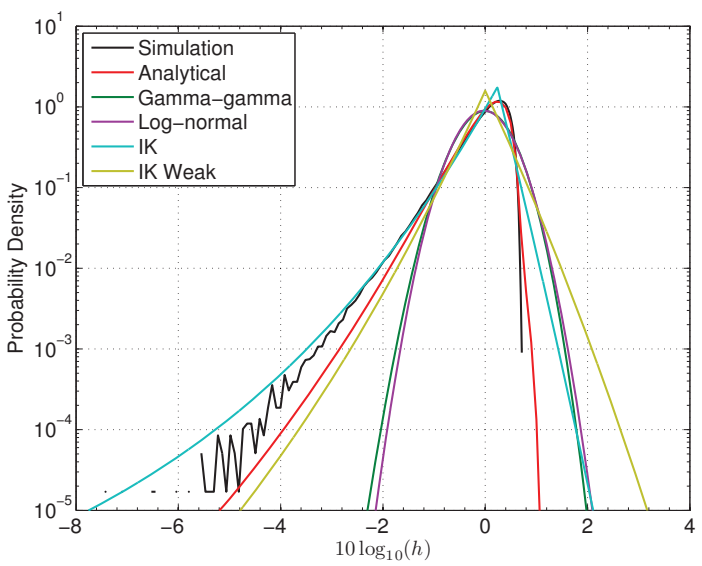

(d) $L=3.2 \mathrm{Km}, D=14 \mathrm{~cm}$

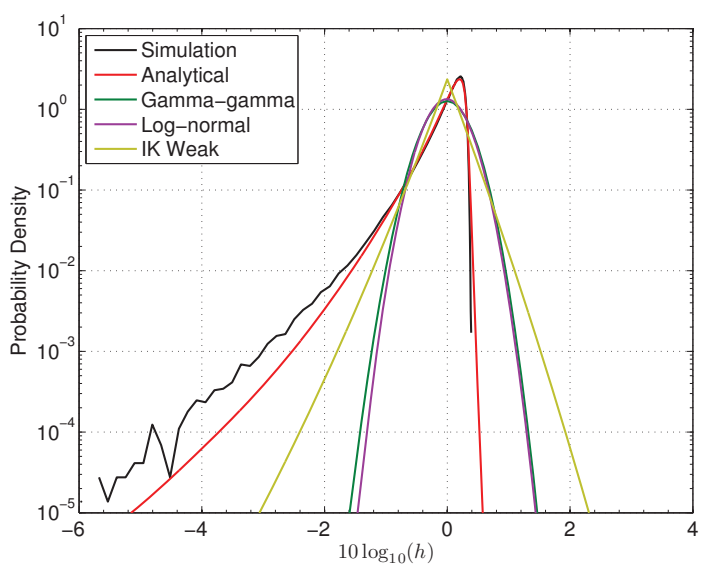

(f) $L=4 \mathrm{Km}, D=20 \mathrm{~cm}$

Figure 4.1: Fading pdf models comparison. Beam wander only pointing error. 


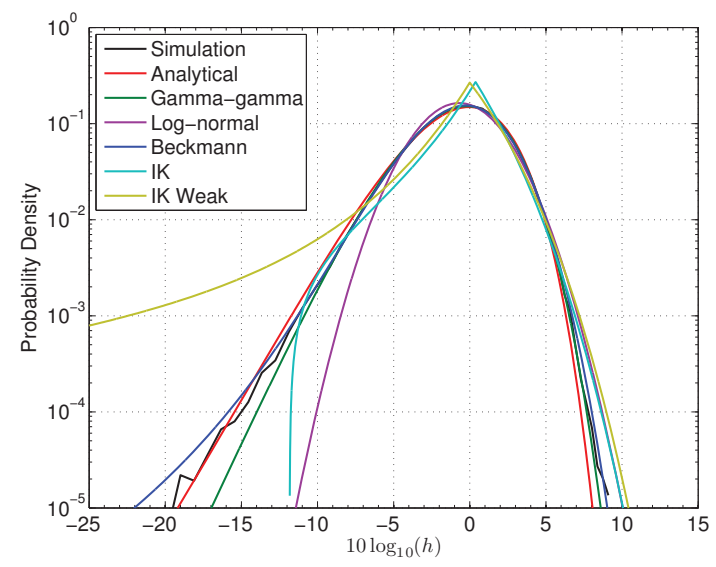

(a) $D=0 \mathrm{~cm}$

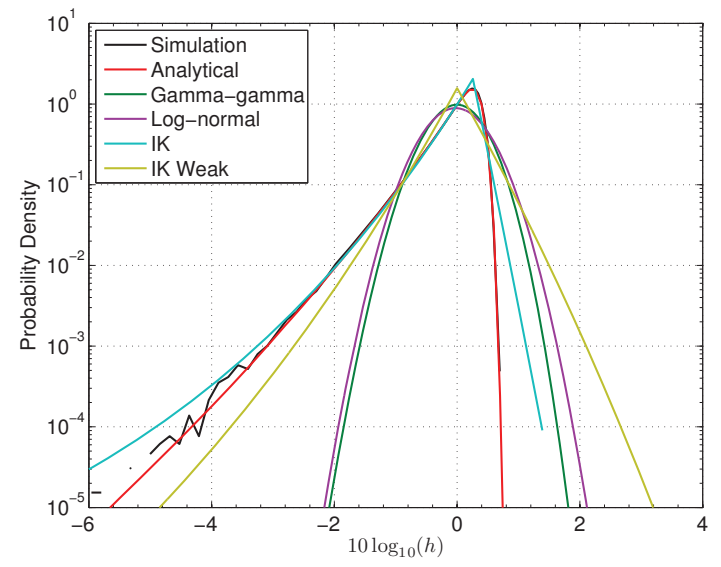

(b) $D=10 \mathrm{~cm}$

Figure 4.2: Fading pdf models comparison. Gaussian extra pointing error. $C_{n}^{2}=$ $1 \times 10^{-13} \mathrm{~m}^{-2 / 3} . L=0.4 \mathrm{Km} . \Delta x=\Delta y=0 \mathrm{~cm} . \sigma_{b}=0.32 \mathrm{~cm} . \sigma_{p}=1.25 \mathrm{~cm} . \sigma_{d}=$ $1.29 \mathrm{~cm} . d_{x}=0.625 \mathrm{~mm}$. Coherence radius is $1.6 \mathrm{~cm}$. Rytov variance is 0.37 . 


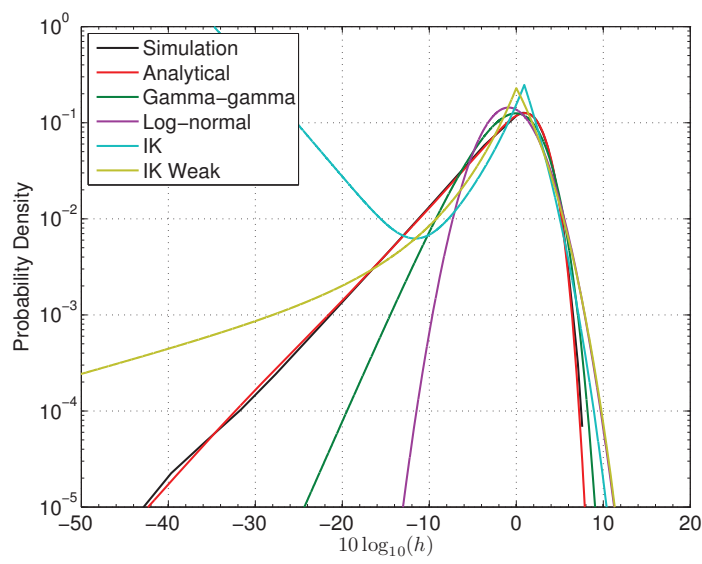

(a) No misalignment, $D=0 \mathrm{~cm}$

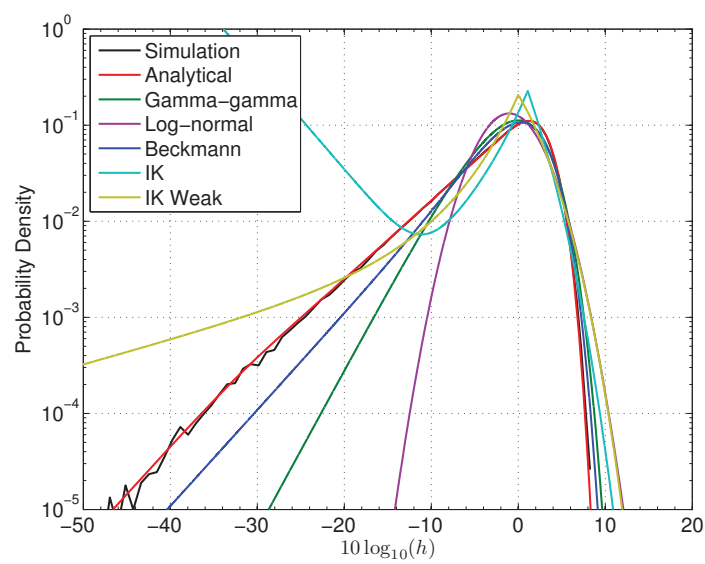

(c) $1.25 \mathrm{~cm}$ misalignment, $D=0 \mathrm{~cm}$

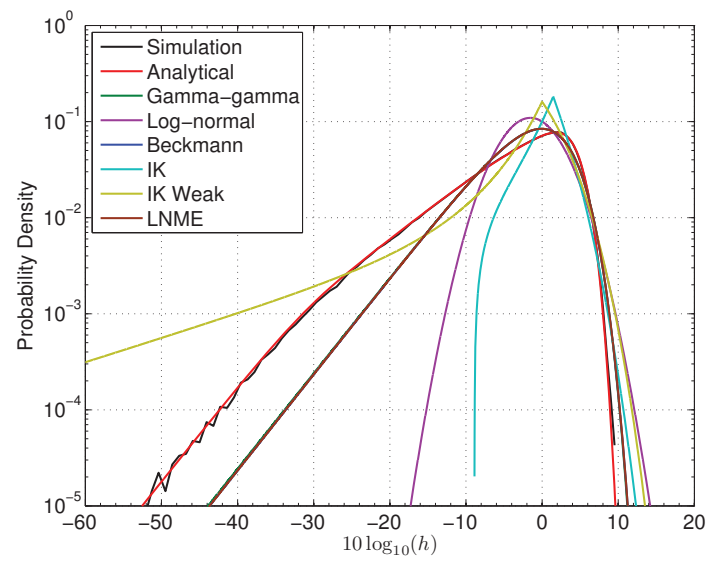

(e) $2.5 \mathrm{~cm}$ misalignment, $D=0 \mathrm{~cm}$

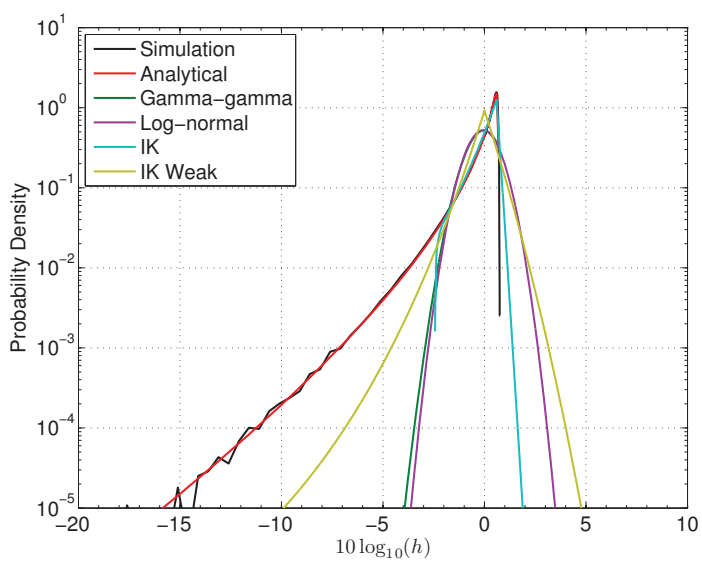

(b) No misalignment, $D=14 \mathrm{~cm}$

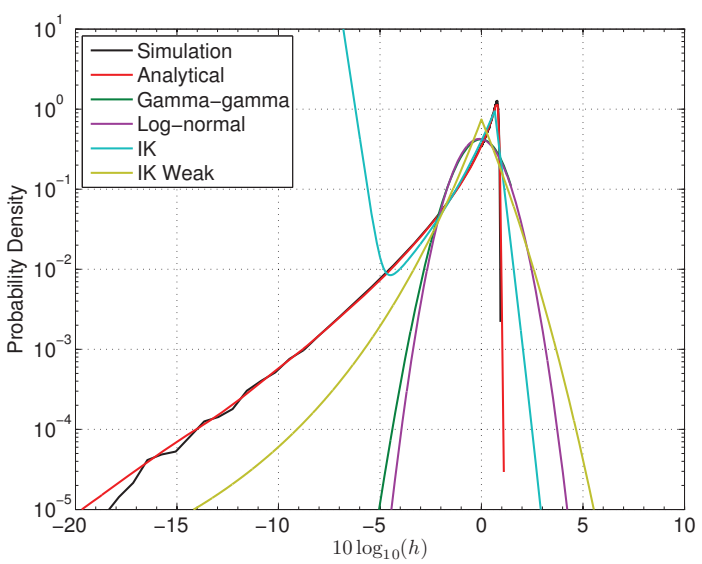

(d) $1.25 \mathrm{~cm}$ misalignment, $D=14 \mathrm{~cm}$

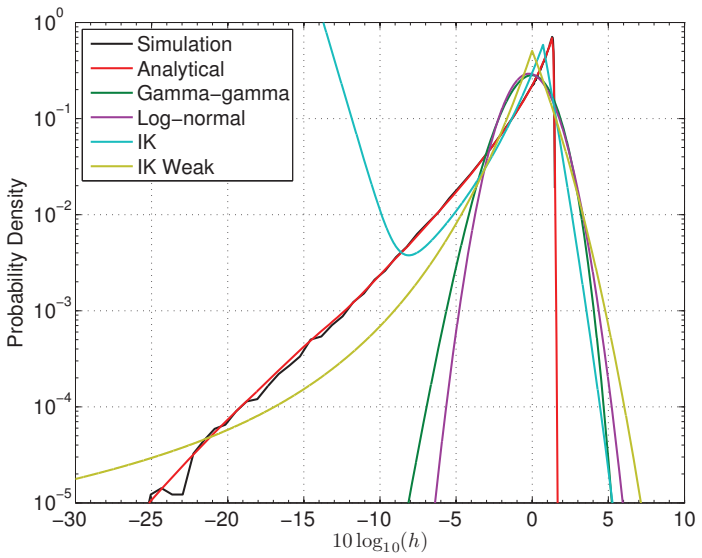

(f) $2.5 \mathrm{~cm}$ misalignment, $D=14 \mathrm{~cm}$

Figure 4.3: Fading pdf models comparison. Gaussian extra pointing error model. $C_{n}^{2}=5 \times 10^{-15} \mathrm{~m}^{-2 / 3} . L=1.6 \mathrm{Km} . \sigma_{b}=0.58 \mathrm{~cm} . \sigma_{p}=2.5 \mathrm{~cm} . \sigma_{d}=2.57 \mathrm{~cm}$. Coherence radius is $4.2 \mathrm{~cm}$. Rytov variance is 0.24 . 


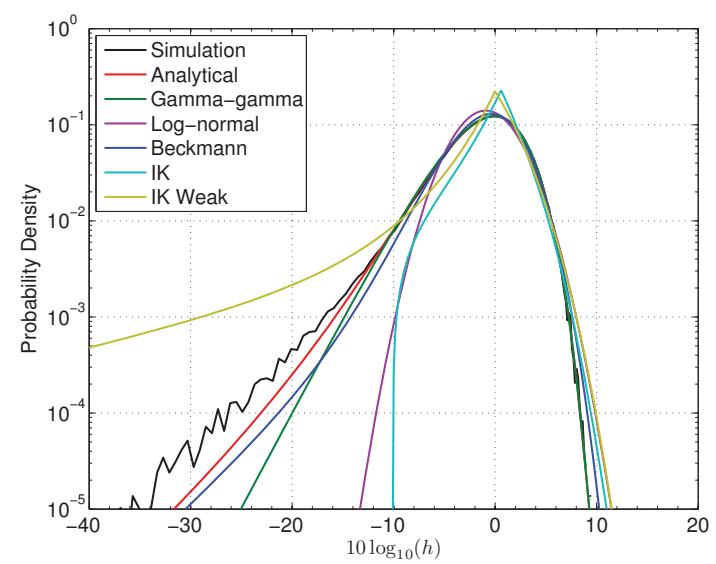

(a) $D=0 \mathrm{~cm}$

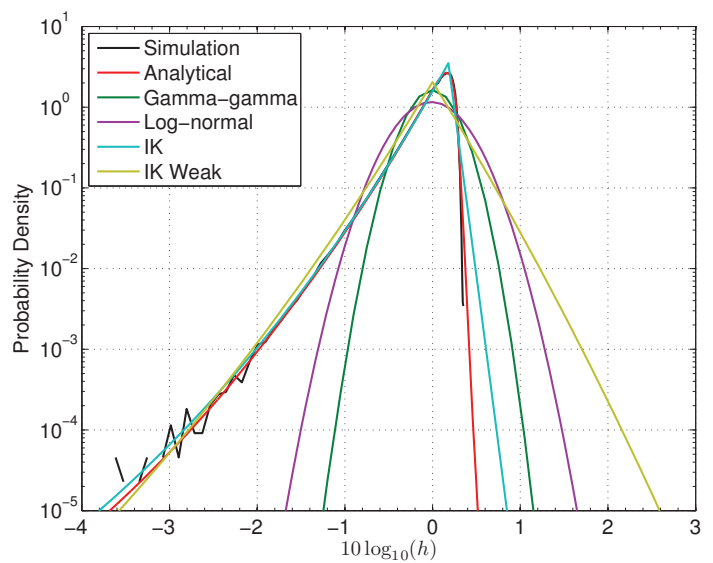

(b) $D=20 \mathrm{~cm}$

Figure 4.4: Fading pdf models comparison. Fast-tracking residual error model. $C_{n}^{2}=5 \times 10^{-15} \mathrm{~m}^{-2 / 3} . L=4 \mathrm{Km} . \Delta x=\Delta y=0 \mathrm{~cm} . \quad \sigma_{d}=2 \mathrm{~cm} . \quad d_{x}=1.6 \mathrm{~mm}$. Coherence radius is $2.4 \mathrm{~cm}$. Rytov variance is 1.27 .

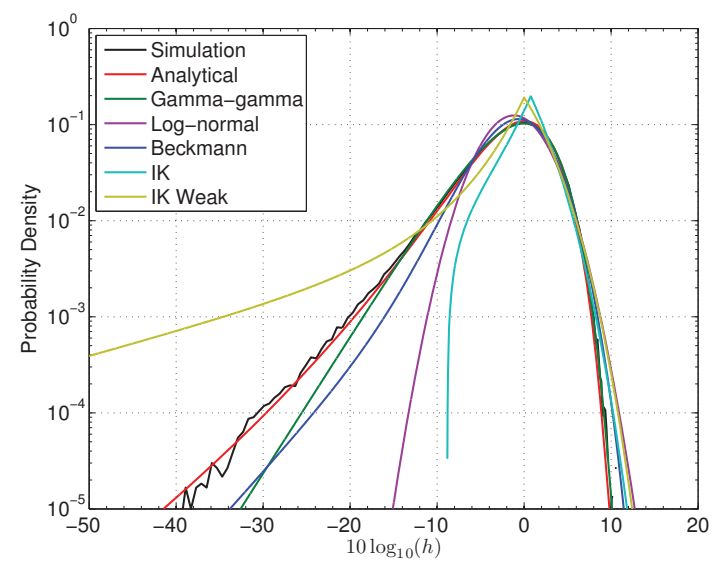

(a) $D=0 \mathrm{~cm}$

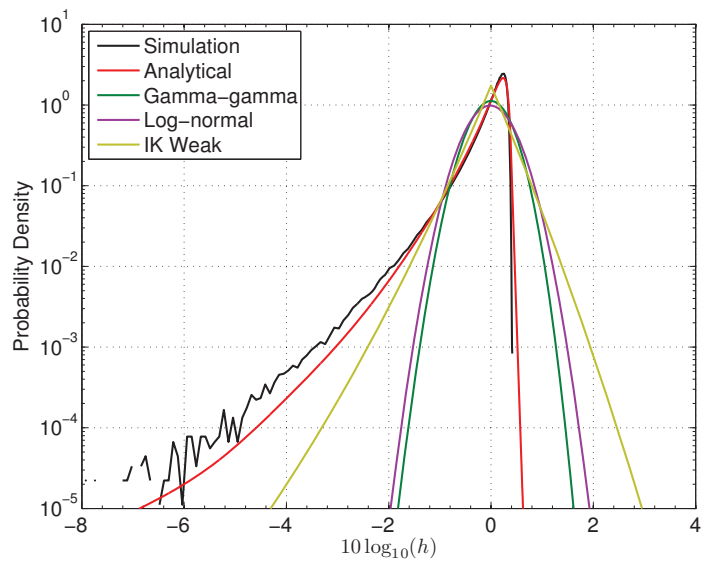

(b) $D=20 \mathrm{~cm}$

Figure 4.5: Fading pdf models comparison. Gaussian extra pointing error model. $C_{n}^{2}=5 \times 10^{-15} \mathrm{~m}^{-2 / 3} . \quad L=4 \mathrm{Km} . \Delta x=\Delta y=0 \mathrm{~cm} . \quad \sigma_{b}=2.29 \mathrm{~cm} . \quad \sigma_{p}=1 \mathrm{~cm}$. $\sigma_{d}=2.5 \mathrm{~cm} . d_{x}=2.5 \mathrm{~mm}$. Coherence radius is $2.4 \mathrm{~cm}$. Rytov variance is 1.27 . 


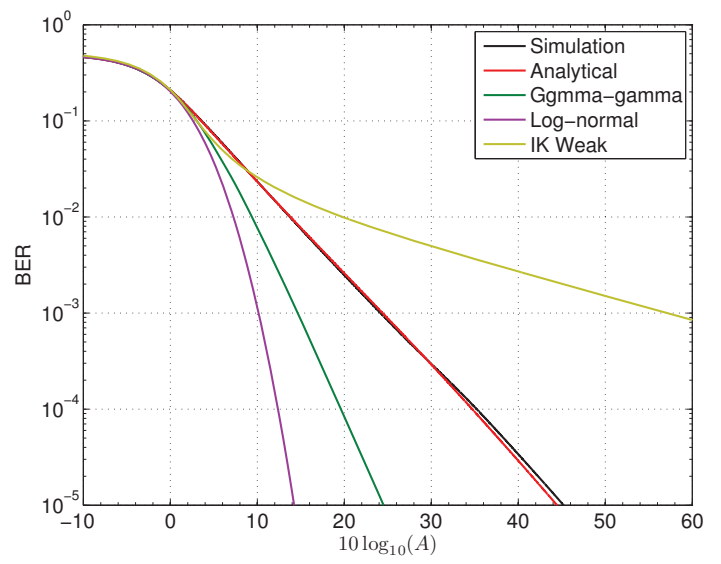

(a) $\Delta x=\Delta y=0 \mathrm{~cm} \cdot D=0 \mathrm{~cm}$

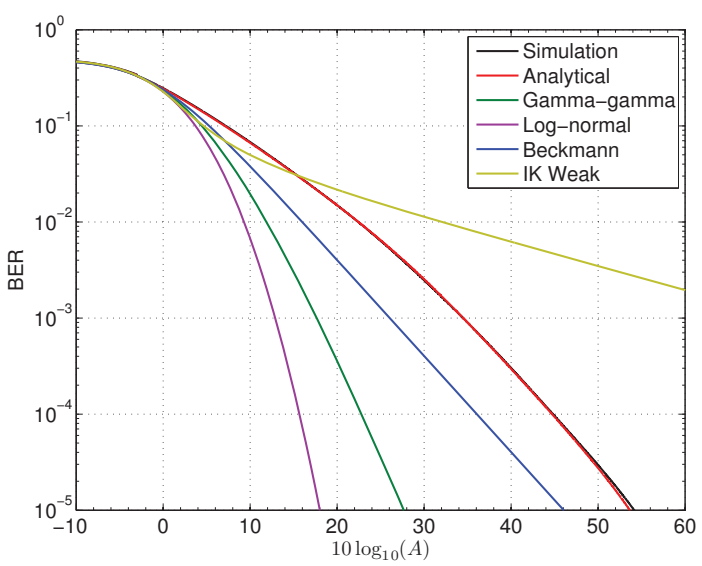

(c) $\Delta x=\Delta y=2.5 \mathrm{~cm} \cdot D=0 \mathrm{~cm}$

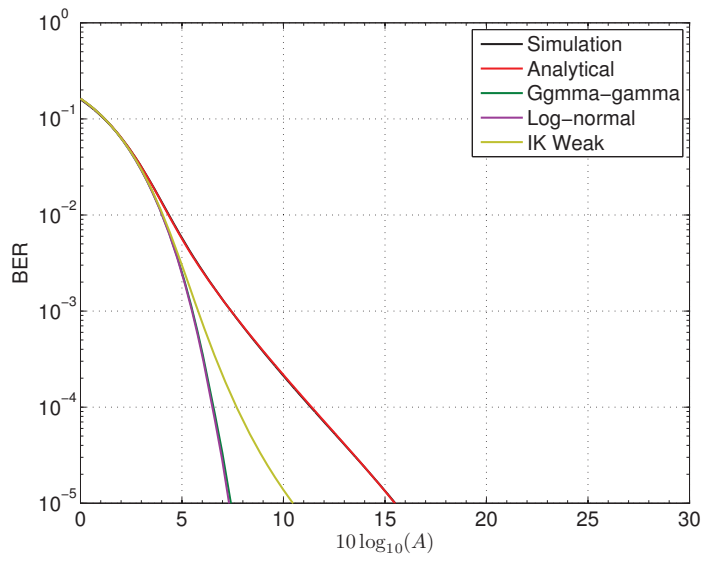

(b) $\Delta x=\Delta y=0 \mathrm{~cm} \cdot D=14 \mathrm{~cm}$

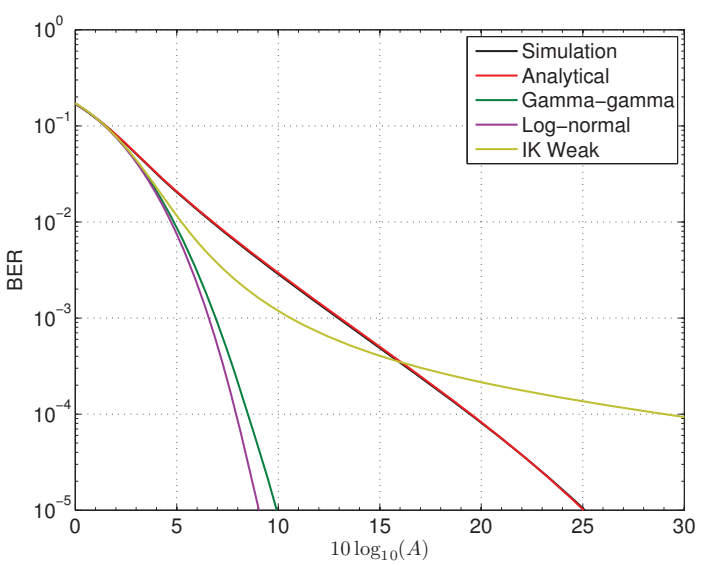

(d) $\Delta x=\Delta y=2.5 \mathrm{~cm} \cdot D=14 \mathrm{~cm}$

Figure 4.6: Average BER comparison. $L=1.6 \mathrm{Km} . C_{n}^{2}=5 \times 10^{-15} \mathrm{~m}^{-2 / 3}$. Gaussian extra pointing error. $\sigma_{b}=0.58 \mathrm{~cm} . \sigma_{p}=2.5 \mathrm{~cm} . \sigma_{d}=2.57 \mathrm{~cm}$. 


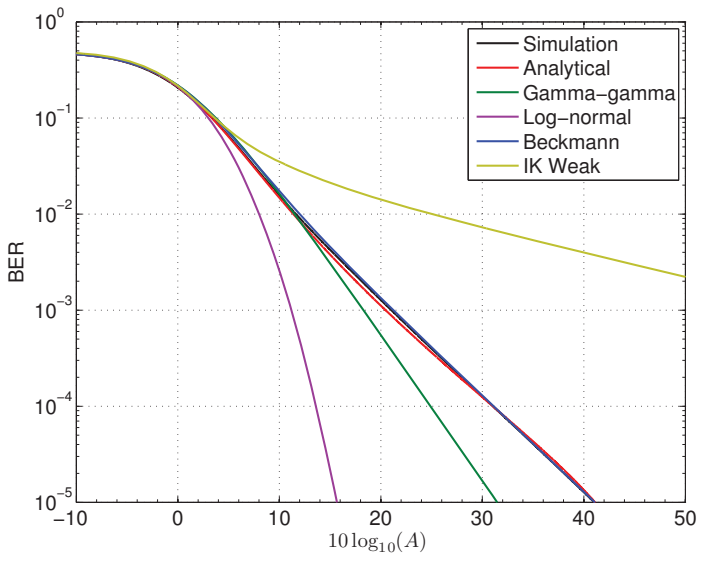

(a) Beam wander only, $D=0 \mathrm{~cm}$

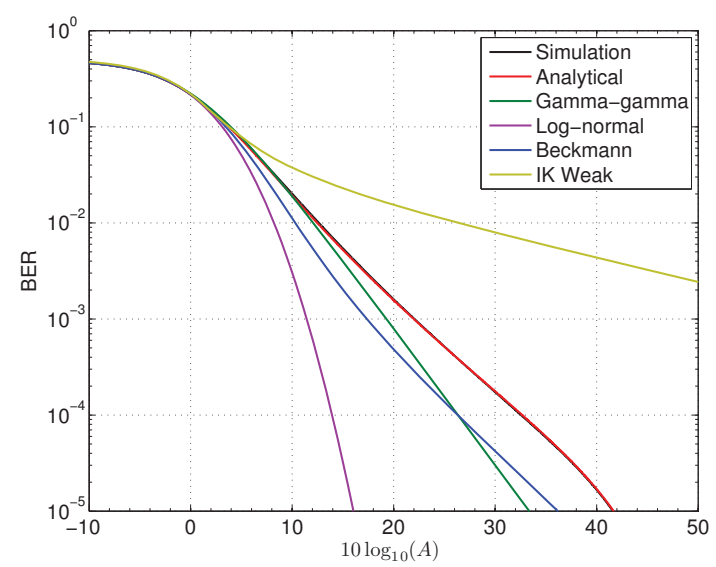

(c) Gaussian extra pointing error, $D=0 \mathrm{~cm}$

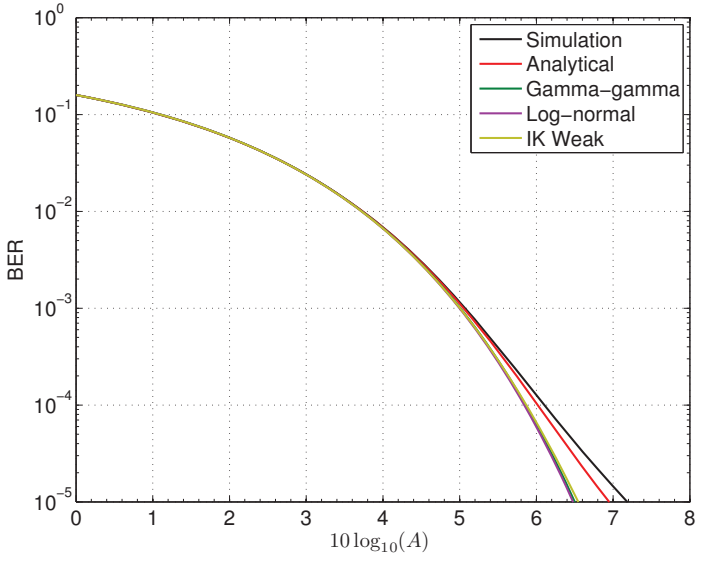

(b) Beam wander only, $D=20 \mathrm{~cm}$

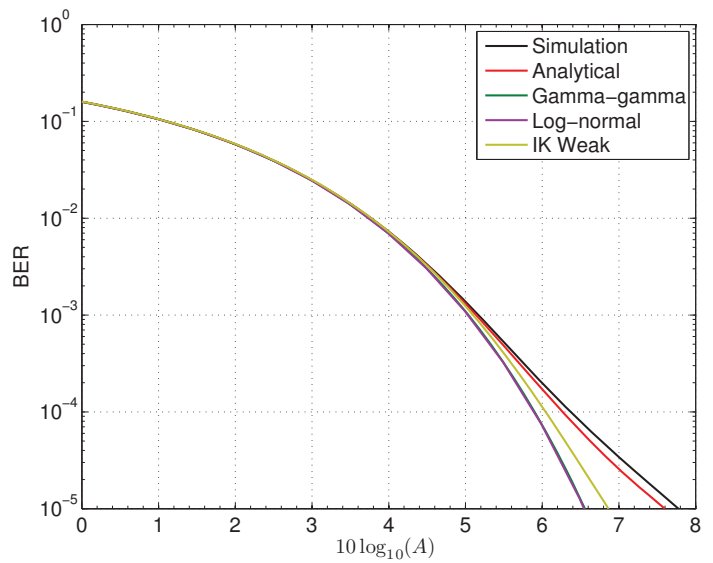

(d) Gaussian extra pointing error, $D=20 \mathrm{~cm}$

Figure 4.7: Average BER comparison. $L=4 \mathrm{Km} . C_{n}^{2}=5 \times 10^{-15} \mathrm{~m}^{-2 / 3}$. Beam wander only pointing error and Gaussian extra pointing error. 


\section{Chapter 5}

\section{Conclusions}

\subsection{Summary of Dissertation}

This thesis studies the fading pdf of the received beam power of the FSO communication system with pointing error under different turbulence strengths, propagation distances, receiver aperture sizes, and beam diverging angles. In the study detailed in chapter 2 , an analytical method is used to calculate the overall fading pdf of the FSO communication system with collimated laser beam and pointing error. In the analytical method, the overall power fading is formulated as the joint effect of the fast-tracked power fading and the pointing error induced average received power fading. Given the fast-tracked beam profile, the fast-tracked on-axis and off-axis fading pdf and the pointing error, the overall fading pdf can be directly calculated. The analytically calculated fading pdfs are then verified by the direct large-scale wave-optics numerical simulations under different turbulence strengths, propagation distances, receiver aperture sizes and pointing errors. In the simulation, the FSO system uses the collimated Gaussian laser beam along a horizontal direction. The laser wavelength is $\lambda=1.54 \times 10^{-6} \mathrm{~m}$. The simulations are performed under different 
turbulence strengths and propagation distances. The refractive index structure parameter ranges from $5 \times 10^{-15} \mathrm{~m}^{-2 / 3}$ to $1 \times 10^{-13} \mathrm{~m}^{-2 / 3}$. The propagation distance ranges from $400 \mathrm{~m}$ to $4000 \mathrm{~m}$. The receiver aperture size ranges from point-like $0 \mathrm{~cm}$ aperture to aperture with $20 \mathrm{~cm}$ diameter. The simulations cover from weak to strong fluctuation regime. To accurately obtain especially the deep fading tail of the fading pdf, the simulations are conducted under large grid size with high resolution. The field grid size $N \times N$ ranges from $1024 \times 1024$ to $2048 \times 2048$. The field resolution $d x$ ranges from $0.625 \mathrm{~mm}$ to $2.5 \mathrm{~mm}$, which is much smaller than the coherence radius in each case. The iteration number ranges from 0.4 million to 1 million. The analytical calculated fading pdfs are also compared with the GG and LN fading pdf models. The system average BER performance and the outage probability estimated by the analytical method, the GG and LN fading pdf models are also compared with that estimated by the direct numerical simulation.

Although the FSO communication system with collimated laser beam is the mostly studied case, previous study by Zhao, Liao and Zhang shows that better system performance can be achieved when the laser beam is transmitted with an optimal diverging angle [1]. The study detailed in chapter 3 expands the analytical method to calculate the overall fading pdf of the FSO communication system when the laser beam is transmitted with beam diverging angle. First, the fast-tracked on-axis and off-axis fading pdfs with point-like receiver aperture are proved to be still well modeled by the gamma pdf model when the laser beam is transmitted with a either focused or diverged beam angle. Then based on the gamma pdf model, the fast-tracked on-axis and off-axis fading variance, the fast-tracked beam profile, and the pointing error model, the overall fading pdf of the FSO communication system with point-like receiver aperture is calculated by the analytical method. Large-scale numerical wave-optics simulations are performed to verify the analytical method in both focused and diverged laser beam cases under different turbulence strengths, propagation distances, and receiver aperture sizes. In the simulations, the laser wavelength is $\lambda=1.54 \times 10^{-6} \mathrm{~m}$. The refractive index structure parameter ranges from $5 \times 10^{-15} \mathrm{~m}^{-2 / 3}$ to $5 \times 10^{-14} \mathrm{~m}^{-2 / 3}$. The propagation distance $L$ ranges from $0.75 \mathrm{Km}$ to $2.5 \mathrm{Km}$ in the focused beam cases. And 
$L$ ranges from $0.5 \mathrm{Km}$ to $2 \mathrm{Km}$ in the diverged beam cases. The receiver aperture is the point-like $0 \mathrm{~cm}$ aperture, which contains only a single pixel in the simulation field. It is much smaller than the coherence radius and can be regarded to be a point in the optical field. To accurately obtain especially the deep fading tail of the fading pdf, the simulations are conducted under large grid size with high resolution. The field grid size ranges from $1024 \times 1024$ to $2048 \times 2048$. The field resolution ranges from $0.625 \mathrm{~mm}$ to $2.5 \mathrm{~mm}$, which is much smaller than the corresponding coherence radius in each case. The iteration number ranges from 0.19 million to 0.62 million to obtain a smooth pdf curve and enough fading pdf tail information.

The study detailed in chapter 4 compares the analytical method with the previously proposed heuristic fading pdf models since 1970s. Both the analytical method and the previously proposed heuristic fading pdf models are compared with the directly simulated fading pdfs under different turbulence strengths, propagation distances, receiver aperture sizes and pointing errors. Also, it should be noted that in the previous studies, the previously proposed heuristic fading pdf models generally only studied the beam wander only pointing error case. In chapter 4, the fading pdf models are studied with both the beam wander only pointing error and the general pointing error cases under different turbulence strengths, propagation distances, and receiver aperture sizes. In both pointing errors, the fading pdfs are simulated with much higher precision than these in the previously published papers where the previously proposed heuristic fading pdf models are proposed and compared. In the simulation, the FSO system uses the collimated Gaussian laser beam along a horizontal direction. The laser wavelength is $\lambda=1.54 \times 10^{-6} \mathrm{~m}$. For the beam wander only pointing error case, the fading pdfs are compared with propagation distance $L=1.6 \mathrm{Km}, L=3.2 \mathrm{Km}$ and $L=4 \mathrm{Km}$. The refractive index structure parameter $C_{n}^{2}=5 \times 10^{-15} \mathrm{~m}^{-2 / 3}$. The receiver aperture size ranges from point-like $0 \mathrm{~cm}$ aperture to aperture with $20 \mathrm{~cm}$ diameter. The Rytov variance ranges from 0.24 to 1.27 . The simulations cover from weak to strong fluctuation regime. The field grid size $N \times N$ ranges from $1024 \times 1024$ to $1536 \times 1536$. The field resolution $d x$ is $1.75 \mathrm{~mm}$ and $2.5 \mathrm{~mm}$, which is much smaller than the corresponding coher- 
ence radius in each case. The phase screen number $N_{p h z}$ ranges from 10 to 12 . The iteration number $M$ ranges from 0.3 million to 0.7 million. For the general pointing error case, both beam wander plus extra pointing error and fast-tracking residual pointing error are studied. The propagation distance are $L=0.4 \mathrm{Km}, L=1.6 \mathrm{Km}$ and $L=4 \mathrm{Km}$. The refractive index structure parameter $C_{n}^{2}=1 \times 10^{-13} \mathrm{~m}^{-2 / 3}$ at $L=0.4 \mathrm{Km}$. $C_{n}^{2}=5 \times 10^{-15} \mathrm{~m}^{-2 / 3}$ at $L=1.6 \mathrm{Km}$ and $L=4 \mathrm{Km}$. The Rytov variance ranges from 0.24 to 1.27 . The simulations cover from weak to strong fluctuation regime. At $L=0.4 \mathrm{Km}$, the pointing error is the beam wander plus extra Gaussian pointing error with mean $\Delta=0 \mathrm{~cm}$ and standard deviation $\sigma_{p}=1.25 \mathrm{~cm}$ in both $x$ and $y$ directions. At $L=1.6 \mathrm{Km}$, pointing error is the beam wander plus extra Gaussian pointing error with three different misalignments. They are $\Delta=0 \mathrm{~cm}, \Delta=1.25 \mathrm{~cm}$ and $\Delta=2.5 \mathrm{~cm}$ in both $x$ and $y$ directions. The standard deviation $\sigma_{p}=2.5 \mathrm{~cm}$ in both $x$ and $y$ directions in all the three misalignment cases. At $L=4 \mathrm{Km}$, the pointing errors are the beam wander plus extra Gaussian pointing error and the fasttracking residual pointing error. In the beam wander plus extra Gaussian pointing error case, $\Delta=0 \mathrm{~cm}$ and $\sigma_{p}=1 \mathrm{~cm}$ in both $x$ and $y$ directions. In the fast-tracking residual pointing error case, $\Delta=0 \mathrm{~cm}$ and the stand deviation of overall pointing error is $\sigma_{d}=2 \mathrm{~cm}$. To accurately obtain especially the deep fading tail of the simulated fading pdf, the simulations are conducted under large grid size with high resolution. The field grid size $N \times N$ ranges from $1024 \times 1024$ to $2048 \times 2048$. The field resolution $d x$ ranges from $0.625 \mathrm{~mm}$ to $2.5 \mathrm{~mm}$, which is much smaller than the corresponding coherence radius in each case. The phase screen number $N_{p h z}$ ranges from 10 to 12 . The iteration number $M$ ranges from 0.4 million to 1 million. The system BER and outage performance estimated by different fading pdf models are compared. Based on the analytical method, the limitation and relationship of the previously proposed heuristic fading models are analyzed. 


\subsection{Summary of Key Results}

In this thesis, the analytical method is used to calculate the overall fading pdf of FSO communication system with pointing error. The analytical method considers three factors, namely, the pointing error, the fast-tracked beam profile and the fast-tracked on-axis and off-axis fading pdfs. The overall fading pdf is formulated as the modulation of the random fading induced by the fast-tracked fading and the random mean induced by the pointing error. The direct large-scale wave-optics simulations show the analytical method perfectly estimates the overall fading pdf with collimated laser beam under different turbulence strengths, propagation distances, receiver aperture sizes and pointing errors. Further, the analytical method is expanded to FSO communication system with beam diverging angle case. It is found the fast-tracked on-axis and off-axis fading pdfs with point-like receiver can also be well modeled by the gamma pdf model when the laser beam is transmitted with a diverging angle. The analytically calculated fading pdf accurately fits the directly simulated fading pdf for both focused and diverged laser beam cases under different propagation distances and turbulence strengths. It is also found that when the laser beam is well focused, the overall fading pdf is affected by both on-axis and off-axis fast-tracked fading pdfs. When the laser beam is largely diverged, overall fading pdf can be approximated by the fast-tracked on-axis fading pdf. The analytical method is also compared with the previously proposed heuristic fading pdf models. Both the turbulence induced beam wander only pointing error and the more general pointing error are considered. It is shown only the analytical method accurately estimates the fading pdf under different turbulence strengths, propagation distances, receiver aperture sizes and pointing errors. For other heuristic fading pdf models, when there is no mechanical induced extra pointing error, and with point-like receiver aperture, Beckmann fading pdf model can well estimate the overall fading pdf. GG fading pdf model can fit the directly simulated fading pdf with turbulence induced beam wander only pointing error and point-like receiver aperture when the beam wander effect is weak. IK, IK Weak and LN fading pdf models are generally different from the directly 
simulated fading pdfs.

\subsection{Future Work}

The analytical method accurately calculates the overall fading pdf of the FSO communication system with pointing error under different turbulence strengths, propagation distances, receiver aperture sizes, beam diverging angles and pointing errors. To calculate the overall fading pdf, the analytical method requires the fast-tracked beam profile and the fast-tracked on-axis and off-axis fading pdfs of the turbulence channel. Current study shows the fasttracked beam profile deviates from both Gaussian distribution model and the diffraction pattern. In this thesis, the fast-tracked beam profile is directly obtained from numerical simulations. For the fast-tracked on-axis and off-axis fading pdfs, Zhao and Liao show that for the FSO communication system with collimated laser beam and point-like receiver aperture, the fast-tracked on-axis and off-axis fading pdfs fit well with the gamma pdf model [57]. This thesis proves that when the laser beam is propagated with beam diverging angle, the fast-tracked on-axis and off-axis fading pdf of the received laser beam power by the point-like receiver can still be well modeled by the gamma pdf model. With the gamma pdf model, the variance of the normalized fading at on-axis and off-axis locations are needed to generate the fast-tracked on-axis and off-axis fading pdfs. Currently these variance information is obtained from numerical simulations. When the receiver aperture grows larger, the fast-tracked on-axis and off-axis fading is the summation of a series of correlated gamma distributed random variables with variance corresponding to the location relative to the fast-tracked beam center. Until now there is only asymptotic model for fading pdfs of the received fast-tracked beam power at on-axis and off-axis locations with large receiver aperture. The fast-tracked on-axis and off-axis fading pdfs with large receiver apertures are directly obtained from numerical simulations in this thesis. Hence one of the future work might involve proposing mathematical models for the fast-tracked beam 
profile and the variance of fast-tracked fading pdfs with point-like receiver aperture. The mathematical models might be studied through both theoretical analysis and numerical simulation. Further, a mathematical method need to be proposed to calculate the fast-tracked on-axis and off-axis fading pdfs with large receiver aperture based on the gamma pdf model of fast-tracked on-axis and off-axis fading pdfs with point-like receiver and the correlation between the fast-tracked fading at different locations. With the mathematical models of these channel information, the overall fading pdf of the FSO communication system with pointing error can be directly calculated from channel parameters.

The analytical method accurately calculates the overall fading pdf under all different situations. Some of the previously proposed heuristic fading pdf models provide close fits to the directly simulated fading pdf only under certain conditions. For example, the GG fading pdf model closely fits the simulated fading pdf of the received beam power by point-like receiver aperture for turbulence induced beam wander only pointing error case when the turbulence is weak. When the turbulence becomes stronger, the receiver aperture grows larger, or the pointing error becomes more general, the GG fading pdf model deviates from the simulated fading pdf. The parameters of the GG fading pdf is claimed to be directly calculated from the turbulence parameter [37]. However, further study shows the GG fading pdf model generated from directly calculated parameters generally deviates from the simulated fading pdf [38]. The analytical method explains that the GG can be regarded to an approximation of the analytical method under certain conditions. And the parameters of the GG pdf model is also able to be derived from the analytical method. Hence another future work may involve proposing the approximation of the analytical method under different channel and pointing error conditions. Based on these approximations, the overall fading pdf is supposed to be directly calculated from the channel and pointing error parameters.

In this thesis, the overall fading pdf of the FSO communication system is accurately calculated by the analytical method. The overall fading pdf represents the statistic property of the received laser beam power over a long period of time. As indicated in chapter 2, the 
atmospheric turbulence channel is a slow fading channel comparing to the transmitted data rate. To better estimate the system performance, the next step of study may involve the research on the temporal power spectral density of the received laser beam power. A mathematical model would be proposed to calculate the coherence time of the received beam power according to the channel parameters such as the propagation distance, turbulence strength, receiver aperture size and laser wavelength. 


\section{References}

[1] Z. Zhao, R. Liao, and Y. Zhang, "Impacts of laser beam diverging angle on free-space optical communications," in Proceedings of 2011 IEEE Aerospace Conference, Big Sky, Montana, USA, Mar. 5-12, 2011, pp. 1-10.

[2] Z. Zhao, R. Liao, S. Lyke, and M. Roggemann, "Direct detection free-space optical communications through atmospheric turbulence," in Proceedings of 2010 IEEE Aerospace Conference, Big Sky, Montana, USA, Mar. 6-13, 2010, pp. 1-9.

[3] R. Gagliardi and S. Karp, Optical Communications, 2nd ed. New York, NY: Wiley, 1995.

[4] L. Andrews, "Free-space laser propagation: atmospheric effects," in 2005 Digest of the LEOS Summer Topical Meetings, Newport Beach, California, USA, Jul. 2005, pp. $3-4$.

[5] S. Bloom, E. Korevaar, J. Schuster, and H. Willebrand, "Understanding the performance of free-space optics [invited]," Journal of Optical Networking, vol. 2, no. 6, pp. 178-200, 2003. [Online]. Available: http://jon.osa.org/abstract.cfm? URI=JON-2-6-178

[6] A. Acampora, "Last mile by laser," Scientific American, vol. 287, no. 1, pp. 32-7, 2002. 
[7] S. Bloom and W. Hartley, "The last-mile solution: hybrid FSO radio," Whitepaper, AirFiber Inc, 2002.

[8] D. Heatley, D. Wisely, I. Neild, and P. Cochrane, "Optical wireless: The story so far," IEEE communications magazine, vol. 36, no. 12, pp. 72-74, 1998.

[9] J. Juarez, A. Dwivedi, A. Mammons, S. Jones, V. Weerackody, and R. Nichols, "Freespace optical communications for next-generation military networks," IEEE Communications Magazine, vol. 44, no. 11, pp. 46-51, 2006.

[10] H. Hemmati, Deep Space Optical Communications. Hoboken, NJ: John Wiley \& Sons, 2006.

[11] L. Andrews, R. Phillips, and C. Hopen, Laser Beam Scintillation with Applications. Bellingham, WA: SPIE Press, 2001.

[12] L. Andrews and R. Phillips, Laser Beam Propagation through Random Media. Bellingham, WA: SPIE Press, 2005.

[13] Z. Zhao, R. Liao, S. Lyke, and M. Roggemann, "Reed-solomon coding for free-space optical communications through turbulent atmosphere," in Proceedings of 2010 IEEE Aerospace Conference, Big Sky, Montana, USA, Mar. 6-13, 2010, pp. 1-12.

[14] X. Zhu and J. Kahn, "Free-space optical communication through atmospheric turbulence channels," IEEE Transactions on Communications, vol. 50, no. 8, pp. 1293$1300,2002$.

[15] — - "Performance bounds for coded free-space optical communications through atmospheric turbulence channels," IEEE Transactions on Communications, vol. 51, no. 8, pp. 1233-1239, 2003.

[16] J. Li and M. Uysal, "Optical wireless communications: system model, capacity and coding," in 2003 IEEE 58th Vehicular Technology Conference, 2003. VTC 2003-Fall., vol. 1, Orlando, Florida, USA, Oct. 2003, pp. 168-172. 
[17] D. Kedar and S. Arnon, "Urban optical wireless communication networks: the main challenges and possible solutions," IEEE Communications Magazine, vol. 42, no. 5, pp. S2-S7, 2004.

[18] J. Anguita, I. Djordjevic, M. Neifeld, and B. Vasic, "Shannon capacities and error-correction codes for optical atmospheric turbulent channels," Journal of Optical Networking, vol. 4, no. 9, pp. 586-601, 2005. [Online]. Available: http://jon.osa.org/ abstract.cfm?URI=JON-4-9-586

[19] K. Kiasaleh, "Performance analysis of free-space on-off-keying optical communication systems impaired by turbulence," in Proceedings of SPIE 4635, SPIE Free-Space Laser Communication Technologies XIV, G. S. Mecherle, Ed., vol. 4635, no. 1. San Jose, CA, USA: SPIE, 2002, pp. 150-161. [Online]. Available: http://link.aip.org/ link/?PSI/4635/150/1

[20] Z. Zhao, "Capacity analysis of free-space optical communication channels with multiple receiver apertures," in Proceedings of 2011 IEEE Aerospace Conference, Big Sky, Montana, USA, Mar. 5-12, 2011, pp. 1-11.

[21] Z. Zhao and R. Liao, "Effects of beam wander on free-space optical communications through turbulent atmosphere," in Proceedings of SPIE 7685-19, SPIE Defense, Security, and Sensing, L. M. W. Thomas and E. J. Spillar, Eds., vol. 7685, no. 1. Orlando, Florida, USA: SPIE, Apr. 5-9, 2010. [Online]. Available: http://link.aip. org/link/?PSI/7685/76850K/1

[22] R. J. Cook, "Beam wander in a turbulent medium: An application of ehrenfest's theorem," Journal of the Optical Society of America, vol. 65, no. 8, pp. 942-948, 1975. [Online]. Available: http://www.opticsinfobase.org/abstract.cfm? URI=josa-65-8-942 
[23] J. H. Churnside and R. J. Lataitis, "Wander of an optical beam in the turbulent atmosphere," Applied Optics, vol. 29, no. 7, pp. 926-930, 1990. [Online]. Available: http://ao.osa.org/abstract.cfm?URI=ao-29-7-926

[24] F. Dios, J. Rubio, A. Rodríguez, and A. Comerón, "Scintillation and beam-wander analysis in an optical ground station-satellite uplink," Applied Optics, vol. 43, no. 19, pp. 3866-3873, 2004.

[25] J. R. Dunphy and J. R. Kerr, "Atmospheric beam-wander cancellation by a fast-tracking transmitter,' Journal of the Optical Society of America, vol. 64, no. 7, pp. 1015-1016, 1974. [Online]. Available: http://www.opticsinfobase.org/abstract. cfm?URI=josa-64-7-1015

[26] G. Cap, H. Refai, and J. Sluss, "Optical tracking and auto-alignment transceiver system," in Digital Avionics Systems Conference, 2008. DASC 2008. IEEE/AIAA 27th, St. Paul, Minnesota, USA, Oct. 2008, pp. 2.B.1-1 -2.B.1-9.

[27] C.-C. Chen, H. Ansari, and J. R. Lesh, "Precision beam pointing for laser communication system using a ccd-based tracker," in Proceeding of SPIE 1949, SPIE Space Guidance, Control, and Tracking, G. E. Sevaston and R. H. Stanton, Eds., vol. 1949, no. 1. Orlando, FL, USA: SPIE, 1993, pp. 15-24. [Online]. Available: http:// link.aip.org/link/?PSI/1949/15/1

[28] L. A. Kranendonk and S. T. Sanders, "Optical design in beam steering environments with emphasis on laser transmission measurements," Applied Optics, vol. 44, no. 31, pp. 6762-6772, 2005. [Online]. Available: http://ao.osa.org/abstract.cfm? $\mathrm{URI}=\mathrm{ao}-44-31-6762$

[29] S. Arnon and N. S. Kopeika, "Effect of particulates on performance of optical communication in space and an adaptive method to minimize such effects," Applied Optics, vol. 33, no. 21, pp. 4930-4937, 1994. [Online]. Available: http://ao.osa.org/ abstract.cfm?URI=ao-33-21-4930 
[30] D. Sadot, A. Melamed, N. Dinur, and N. Kopeika, "Effects of aerosol forward scatter on the long-and short-exposure atmospheric coherence diameter," Waves in Random and Complex Media, vol. 4, no. 4, pp. 487-498, 1994.

[31] G. Parry and P. N. Pusey, "K distributions in atmospheric propagation of laser light," Journal of the Optical Society of America, vol. 69, no. 5, pp. 796-798, 1979. [Online]. Available: http://www.opticsinfobase.org/abstract.cfm?URI=josa-69-5-796

[32] R. L. Phillips and L. C. Andrews, "Universal statistical model for irradiance fluctuations in a turbulent medium," Journal of the Optical Society of America, vol. 72, no. 7, pp. 864-870, 1982. [Online]. Available: http://www.opticsinfobase. org/abstract.cfm?URI=josa-72-7-864

[33] J. H. Churnside and R. J. Hill, "Probability density of irradiance scintillations for strong path-integrated refractive turbulence," Journal of the Optical Society of America A, vol. 4, no. 4, pp. 727-733, 1987. [Online]. Available: http://josaa.osa. org/abstract.cfm?URI=josaa-4-4-727

[34] J. H. Churnside and S. F. Clifford, "Log-normal rician probability-density function of optical scintillations in the turbulent atmosphere," Journal of the Optical Society of America A, vol. 4, no. 10, pp. 1923-1930, 1987. [Online]. Available: http://josaa. osa.org/abstract.cfm?URI=josaa-4-10-1923

[35] R. J. Hill and R. G. Frehlich, "Probability distribution of irradiance for the onset of strong scintillation," Journal of the Optical Society of America A, vol. 14, no. 7, pp. 1530-1540, 1997. [Online]. Available: http://josaa.osa.org/abstract.cfm? URI=josaa-14-7-1530

[36] J. H. Churnside and R. G. Frehlich, "Experimental evaluation of log-normally modulated rician and ik models of optical scintillation in the atmosphere," Journal of the Optical Society of America A, vol. 6, no. 11, pp. 1760-1766, 1989. [Online]. Available: http://josaa.osa.org/abstract.cfm?URI=josaa-6-11-1760 
[37] M. A. Al-Habash, L. C. Andrews, and R. L. Phillips, "Mathematical model for the irradiance probability density function of a laser beam propagating through turbulent media," Optical Engineering, vol. 40, no. 8, pp. 1554-1562, 2001. [Online]. Available: http://link.aip.org/link/?JOE/40/1554/1

[38] F. S. Vetelino, C. Young, L. Andrews, and J. Recolons, "Aperture averaging effects on the probability density of irradiance fluctuations in moderate-to-strong turbulence," Applied Optics, vol. 46, no. 11, pp. 2099-2108, 2007. [Online]. Available: http://ao. osa.org/abstract.cfm?URI=ao-46-11-2099

[39] L. C. Andrews and R. L. Phillips, "I-K distribution as a universal propagation model of laser beams in atmospheric turbulence," Journal of the Optical Society of America A, vol. 2, no. 2, pp. 160-163, 1985. [Online]. Available: http://josaa.osa.org/abstract. cfm?URI=josaa-2-2-160

[40] S. M. Flatté, C. Bracher, and G.-Y. Wang, "Probability-density functions of irradiance for waves in atmospheric turbulence calculated by numerical simulation," Journal of the Optical Society of America A, vol. 11, no. 7, pp. 2080-2092, 1994. [Online]. Available: http://josaa.osa.org/abstract.cfm?URI=josaa-11-7-2080

[41] — - "Probability-density functions of irradiance for waves in atmospheric turbulence by numerical simulation: erratum," Journal of the Optical Society of America A, vol. 12, no. 1, pp. 184-184, 1995. [Online]. Available: http://josaa.osa. org/abstract.cfm?URI=josaa-12-1-184

[42] A. Majumdar, "Free-space laser communication performance in the atmospheric channel," Journal of Optical and Fiber Communications Reports, vol. 2, no. 4, pp. 345-396, 2005.

[43] P. Beckmann, Probability in Communication Engineering. New York, NY: Harcourt, Brace \& World, 1967. 
[44] R. Hill, R. Frehlich, and W. Otto, The Probability Distribution of Irradiance Scintillation.

[45] S. Arnon, "Effects of atmospheric turbulence and building sway on optical wireless-communication systems," Optics Letters, vol. 28, no. 2, pp. 129-131, 2003. [Online]. Available: http://ol.osa.org/abstract.cfm?URI=ol-28-2-129

[46] S. Arnon, S. Rotman, and N. S. Kopeika, "Beam width and transmitter power adaptive to tracking system performance for free-space optical communication," Applied Optics, vol. 36, no. 24, pp. 6095-6101, 1997. [Online]. Available: http://ao. osa.org/abstract.cfm?URI=ao-36-24-6095

[47] S. Arnon and N. S. Kopeika, "Adaptive suboptimum detection of an optical pulse-position-modulation signal with a detection matrix and centroid tracking," Journal of the Optical Society of America A, vol. 15, no. 2, pp. 443-448, 1998. [Online]. Available: http://josaa.osa.org/abstract.cfm?URI=josaa-15-2-443

[48] S. Arnon, "Power versus stabilization for laser satellite communication," Applied Optics, vol. 38, no. 15, pp. 3229-3233, 1999. [Online]. Available: http://ao.osa.org/ abstract.cfm?URI=ao-38-15-3229

[49] H. Eklund, "Signal distortion due to beam-pointing error in a chopper modulated laser system," Applied Optics, vol. 17, no. 2, pp. 289-295, 1978. [Online]. Available: http://ao.osa.org/abstract.cfm?URI=ao-17-2-289

[50] A. A. Farid and S. Hranilovic, "Outage probability for free-space optical systems over slow fading channels with pointing errors," in 19th Annual Meeting of the IEEE Lasers and Electro-Optics Society, 2006. LEOS 2006., Montreal, QC, Canada, Oct. 2006, pp. 82-83.

[51] H. G. Sandalidis, T. Tsiftsis, and G. K. Karagiannidis, "Optical wireless communications with heterodyne detection over turbulence channels with pointing 
errors," Journal of Lightwave Technology, vol. 27, no. 20, pp. 4440-4445, 2009. [Online]. Available: http://jlt.osa.org/abstract.cfm?URI=JLT-27-20-4440

[52] H. Sandalidis, T. Tsiftsis, G. Karagiannidis, and M. Uysal, "BER performance of FSO links over strong atmospheric turbulence channels with pointing errors," Communications Letters, IEEE, vol. 12, no. 1, pp. 44-46, 2008.

[53] A. Farid and S. Hranilovic, "Outage capacity optimization for free-space optical links with pointing errors," Journal of Lightwave Technology, vol. 25, no. 7, pp. 1702 $1710,2007$.

[54] J. Proakis and M. Salehi, Digital Communications, 4th ed. New York, NY: McGrawhill, 2001.

[55] W. Feller, An Introduction to Probability Theory and Its Applications, 2nd ed. Princeton, NJ: Wiley, 1991, vol. 2.

[56] J. C. Camparo, "Stellar scintillation and the atmospheres vertical turbulence profile," Journal of the Optical Society of America A, vol. 18, pp. 631-637, 2001.

[57] Z. Zhao and R. Liao, "On fading probability density functions of fast-tracked and untracked free-space optical communication channels," in Proceedings of SPIE 7924, SPIE Atmospheric and Oceanic Propagation of Electromagnetic Waves V, O. Korotkova, Ed., vol. 7924, no. 1. San Francisco, California, USA: SPIE, Jan. 25, 2011. [Online]. Available: http://link.aip.org/link/?PSI/7924/79240H/1 


\section{Index}

Average BER, 66, 102, 103

Beam broadening effect, 37, 81

Beam radius, 80

Beam wander, 42, 50, 81

Beam wander only pointing error, 46, 96

Beam wander plus extra pointing error, 47 , 48

Beam wave length, 43

Channel information, 54, 55

Coherence radius, 54

Doubly stochastic model, 26

Extra pointing error, 46, 100

Gaussian extra pointing error, 51

Sine extra pointing error, 52

Uniform extra pointing error, 51

Fading pdf model

Analytical Method, 44

Beckmann (LNMR), 24, 32, 95

Gamma-gamma, 24, 34

IK, 24, 29, 95, 103

IK Weak, 97
K, 24, 28, 95

LNME, 24, 31, 95

Log-normal, 24, 26

Fast-tracked beam profile, 42

Fast-tracked fading pdf, 42, 79, 83, 84

Free-space optical (FSO) communication, 41

Gamma pdf model, 43, 79

General pointing error, 99

Kolmogorov spectrum, 53

Misalignment, 42

Optimal beam diverging angle, 74

Outage probability, 69

Overall displacement, 44

Phase screen, 53

Pointing error, 42

Beam wander plus extra pointing error, 42

Fast-tracking residual error, 42, 46, 47, 100

Pointing error sources, 42

Research approach 
Numerical simulation, 25, 42

Physical experiment, 25

Theoretical analysis, 25

Rytov variance, 54

Tracking

Fast-tracking, 42

Slow-tracking, 42

Wave front distortion, 79 